\title{
Polymorphic Relaxed Noninterference
}

\author{
Raimil Cruz \\ PLEIAD Lab, Computer Science Department (DCC) \\ University of Chile \\ Santiago, Chile \\ racruz@dcc.uchile.cl
}

\author{
Éric Tanter \\ PLEIAD Lab, Computer Science Department (DCC) \\ University of Chile, Santiago, Chile \\ \& Inria Paris, France \\ etanter@dcc.uchile.cl
}

\begin{abstract}
Information-flow security typing statically preserves confidentiality by enforcing noninterference. To address the practical need of selective and flexible declassification of confidential information, several approaches have developed a notion of relaxed noninterference, where security labels are either functions or types. The labels-as-types approach to relaxed noninterference supports expressive declassification policies, including recursive ones, via a simple subtyping-based ordering, and provides a local, modular reasoning principle. In this work, we extend this expressive declassification approach in order to support polymorphic declassification. First, we identify the need for bounded polymorphism through concrete examples. We then formalize polymorphic relaxed noninterference in a typed object-oriented calculus, using a step-indexed logical relation to prove that all well-typed terms are secure. Finally, we address the case of primitive types, which requires a form of ad-hoc polymorphism. Therefore, this work addresses practical hurdles to providing controlled and expressive declassification for the construction of information-flow secure systems.
\end{abstract}

\section{INTRODUCTION}

An information-flow security type system statically ensures that public outputs (e.g. String $\mathrm{L}_{\mathrm{L}}$ cannot depend on secret inputs (e.g. String ${ }_{H}$ ), a property known as noninterference (NI) [1]. NI provides a modular reasoning principle about security, indexed by the observational power of an adversary. For instance, a function $f:$ String $_{H} \rightarrow$ String $_{L}$ does not reveal any information about its argument; in fact, in a pure language, it is necessarily a constant function.

But noninterference is too strict in practice: for a system to be useful, confidential information sometimes needs to be declassified. Beyond introducing a declassification operator in the language, which compromises formal reasoning, various approaches have explored structured ways to support declassification policies [2, 3, 4, 5]. In particular, $\mathrm{Li}$ and Zdancewic [3] introduce relaxed noninterference, supporting expressive declassification policies via security labels as functions. In this approach, instead of having security labels such as $\mathrm{H}$ for private and $L$ for public information that are drawn from a fixed lattice of symbols, security labels are the very functions that describe how a given secret can be manipulated in order to produce a public value: for instance, one can realize the declassification policy "only the result of comparing the hash

This work is partially funded by CONICYT FONDECYT Regular Projects 1150017 and 1190058 , and is in part supported by the European Research Council under ERC Starting Grant SECOMP (715753). Raimil Cruz is partially funded by CONICYT-PCHA/Doctorado Nacional/2014-63140148 of the secret string $s$ with a public guess can be made public" by attaching to $s$ a function that implements this declassification $(\lambda x . \lambda y$. hash $(x)=y)$. Any use of the secret that does not follow the declassification policy yields private results. One can express the standard label $\mathrm{H}$ (resp. L) as a constant function (resp. the identity function). A challenging aspect of this approach is that label ordering relies on a semantic interpretation of declassification functions.

A more practical approach than this labels-as-functions approach was recently developed by Cruz et al. [5] in an objectoriented setting, with a labels-as-types perspective: security types are faceted types of the form $T \triangleleft U$ where the first facet $T$-called the safety type - represents the implementation type, exposed to the private observer, and the second facet $U-$ called the declassification type-represents the declassification policy as an object interface exposed to the public observer ${ }^{1}$ For instance, the type String $\triangleleft T$, where $T$ is the empty object interface, denotes private values (no method is declassified) and the type String $\triangleleft$ String represents public values (all methods are declassified). These security types are abbreviated as String $_{H}$ and String ${ }_{L}$, respectively. Interesting declassification policies stand in between these two extremes: for instance, given the interface StringLen $\triangleq$ [length : Unit $\left.t_{L} \rightarrow \operatorname{Int}_{L}\right]$, the faceted type String $\triangleleft$ StringLen exposes the method length to declassify the length of a string as a public integer, but not its content. This type-based approach to declassification is expressive as well as simple-in particular, because labels are types, label ordering is simply subtyping. Also, it extends the modular reasoning principle of NI to account for declassification [5], a property named type-based relaxed noninterference (TRNI). For instance, with TRNI one can prove that a function of type String $\triangleleft$ StringLen $\rightarrow$ Bool $_{\llcorner}$must produce equal results for strings of equal lengths.

The labels-as-types approach of Cruz et al. however lacks security label polymorphism. Security label polymorphism is a very useful feature of practical security-typed languages such as JIF [6] and FlowCaml [7], which has only been explored in the context of standard security labels (symbols from a lattice). To the best of our knowledge, polymorphism has not been studied for expressive declassification mechanisms, such as labels-as-functions [3] or labels-as-types [5]. We extend the

\footnotetext{
${ }^{1}$ To account for $k>2$ observation levels, faceted types can be extended to have $k$ facets [5]. Here, we restrict the presentation to two observation levels
} 
labels-as-types approach with declassification polymorphism, specifically bounded polymorphism that specifies both a lower and an upper bound for a polymorphic declassification type.

The main contribution of this paper is to develop the theory of bounded polymorphic declassification as an extension of TRNI, called polymorphic relaxed noninterference (PRNI for short). PRNI brings new benefits in the expressiveness and design of declassification interfaces. Additionally, we address the necessary support for primitive types, through a form of ad-hoc polymorphism.

The labels-as-types approach has the practical benefits of relying on concepts that are well-known to developers-object interfaces and subtyping -in order to build systems with information flow security that cleanly account for controlled and expressive declassification. This work addresses the two major shortcomings of prior work in order to bring this approach closer to real-world secure programming.

Section III provides background on labels-as-types and TRNI. Section III] then explains the main aspects of polymorphic relaxed noninterference (PRNI). Section IV] formalizes polymorphic declassification in a core object-oriented language $\mathrm{Ob}_{\mathrm{SEC}}^{\langle\rangle}$. Then, Section $\mathrm{V}$ develops a logical relation for PRNI and shows that all well-typed $\mathrm{Ob}_{\mathrm{SEC}}^{\langle\rangle}$terms satisfy PRNI. Section VI extends $\mathrm{Ob}_{\mathrm{SEC}}^{\langle\rangle}$with primitive types. Section VII discusses related work and Section VIII concludes. We have implemented an interactive prototype of $\mathrm{Ob}_{S E C}^{\langle\rangle}$that is available at https://pleiad.cl/gobsec/

\section{BACKGROUND:TYPE-BASED RELAXED NONINTERFERENCE}

We start with a quick review of type-based relaxed noninterference [5]. Faceted security types allow programmers to express declassification policies as type interfaces. For instance, one can express that a login function can reveal the result of comparing a secret password for equality with a public guess.

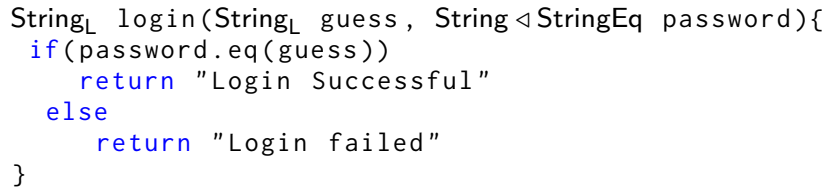

Note that leaking the secret password by directly returning it would not typecheck, since StringEq is not a subtype of String (recall that String $L_{L}$ is short for String $\triangleleft$ String). Taking advantage of the fact that object types are recursive, one can also express recursive declassification, for instance that a list of secret strings can only be declassified by comparing its elements for equality. Likewise, one can express progressive declassification by nesting type interfaces. For instance, assuming that String has a method hash : Unit $_{L} \rightarrow \operatorname{Int}_{\mathrm{L}}$, we can specify that only the hash of the password can be compared for equality with the interface type StringHashEq $\triangleq\left[\right.$ hash: Unit $t_{L} \rightarrow \operatorname{Int} \triangleleft \operatorname{IntEq}$, where $\operatorname{lntEq} \triangleq\left[\right.$ eq : Int $\mathrm{L}_{\mathrm{L}} \rightarrow$ Bool $\left._{\mathrm{L}}\right]:$

String $L_{L} \operatorname{login}\left(\operatorname{Int}_{\mathrm{L}}\right.$ guess, String $\triangleleft$ StringHashEq password)\{

$$
\begin{aligned}
& e::=v|e . m(e)| x \quad \text { (terms) } \\
& v::=[z: S \Rightarrow \overline{m(x) e} \quad \text { (values) } \\
& T, U::=O \mid \alpha \quad \text { (types) } \\
& O::=\operatorname{Obj}(\alpha) \cdot[\overline{m: S \rightarrow S}] \text { (object types) } \\
& S::=T \triangleleft U \quad \text { (security types) } \\
& \Gamma \vdash e: S \quad \Gamma::=\bullet \mid \Gamma, x: S \text { (type environment) } \\
& (\mathrm{TmD}) \frac{\begin{array}{cc}
\Gamma \vdash e_{1}: T \triangleleft U & m \in U \\
\operatorname{msig}(U, m)=S_{1} \rightarrow S_{2} & \Gamma \vdash e_{2}: S_{1}
\end{array}}{\Gamma \vdash e_{1} \cdot m\left(e_{2}\right): S_{2}} \\
& \begin{array}{cc}
\Gamma \vdash e_{1}: T \triangleleft U & m \notin U \\
(\mathrm{TmH}) \frac{c}{\operatorname{msig}(T, m)=S_{1} \rightarrow T_{2} \triangleleft U_{2}} \quad \Gamma \vdash e_{2}: S_{1} \\
\hline \Gamma \vdash e_{1} \cdot m\left(e_{2}\right): T_{2} \triangleleft \top
\end{array}
\end{aligned}
$$

Fig. 1. Ob $b_{\mathrm{SEC}}$ : Syntax and Static semantics (excerpts from [5])

if (password.hash (). eq(guess)) ...

Cruz et al. formalize faceted security types in $\mathrm{Ob}_{\mathrm{SEC}}$, a core object-oriented language with three kinds of expressions: variables, objects and method invocations (Figure 11). An object $[z: S \Rightarrow \overline{m(x) e}]$ is a collection of methods that can refer to the defining object with the self variable $z$. An object type $\operatorname{Obj}(\alpha) \cdot[m: S \rightarrow S]$ is a collection of method signatures that have access to the defining type through the self type variable $\alpha$. Security types $S=T \triangleleft U$ are composed of two object types $T$ and $U$. Note that to be well-formed, a security type $T \triangleleft U$ requires $U$ to be a supertype of $T$. The type abstraction mechanism of subtyping (by which a supertype "hides" members of its subtypes) is the key element to express declassification.

The ObSEC type system defines two rules to give a type to a method invocation depending on whether the invoked method is in the declassification type or not. Rule (TmD) specifies that if the invoked method $m$ is in the declassification type $U$ with type $S_{1} \rightarrow S_{2}$, then the result type of the method invocation expression is $S_{2}$. Conversely, if the method $m$ is only present in the safety type $T$ with type $S_{1} \rightarrow T_{2} \triangleleft U_{2}$, then the result type of the method invocation is $T_{2} \triangleleft \top$ (TmH): if we bypass the declassification type, the result must be protected as a secret.

The security property obtained by this approach is called Type-based Relaxed Noninterference (TRNI). At the core of TRNI is a notion of observational equivalence between objects up to the discrimination power of the public observer, which is specified by the declassification type. More precisely, two objects $o_{1}$ and $o_{2}$ are equivalent at type $T \triangleleft U$ if, for any method $m$ with type $S_{1} \rightarrow S_{2}$ in the declassification type $U$, invoking $m$ with equivalent values $v_{1}$ and $v_{2}$ at type $S_{1}$, produces equivalent results at type $S_{2}$.

TRNI is formulated as a modular reasoning principle, over open terms: $\operatorname{TRNI}(\Gamma, e, S)$. The closing typing environment 
$\Gamma$ specifies the secrecy of the inputs that $e$ can use, and the security type $S$ specifies the observation power of the adversary on the output.

For instance, suppose StringLen is an interface that exposes a length: Unit $\rightarrow \operatorname{Int}_{\mathrm{L}}$ method. Then, with $\Gamma=$ $x$ : String $\triangleleft$ StringLen, the judgment $\operatorname{TRNI}\left(\Gamma\right.$, $x$.length ()$\left., \operatorname{lnt} \mathrm{L}_{\mathrm{L}}\right)$ implies: given the knowledge that two input strings $v_{1}$ and $v_{2}$ have the same length, the lower observer does not learn anything new about the inputs by executing x.length(). Conversely, TRNI( $\Gamma$, x.eq("a"), Bool $\left.\mathrm{L}_{\mathrm{L}}\right)$ does not hold: executing x.eq("a") and exposing the result as a public value would reveal more information than permitted by the input declassification type (eq $\notin$ StringLen). However, $\operatorname{TRNI}\left(\Gamma\right.$, x.eq("a"), Bool $\left.{ }_{H}\right)$ does hold, because the result is private and therefore unaccessible to the public observer.

\section{Polymorphic Relaxed Noninterference}

We first motivate polymorphic declassification with faceted types, and then we illustrate the role of bounded polymorphism for declassification. Finally, we give an overview of the modular reasoning principle of polymorphic relaxed noninterference.

\section{A. Polymorphic Declassification}

When informally discussing the possible extensions to their approach to declassification, Cruz et al. [5] illustrate the potential benefits of polymorphic declassification by giving the example of a list of strings that is polymorphic with respect to the declassification type of its elements:

$$
\begin{aligned}
\text { ListStr }\langle X\rangle \triangleq & {\left[\text { isEmpty : Unit } \mathrm{L}_{\mathrm{L}} \rightarrow \text { Bool }_{\mathrm{L}},\right.} \\
& \text { head : Unit } \mathrm{L} \rightarrow \text { String } \triangleleft X, \\
& \text { tail : Unit } \left.t_{\mathrm{L}} \rightarrow \text { ListStr }\langle X\rangle_{\mathrm{L}}\right]
\end{aligned}
$$

This recursive polymorphic declassification policy allows a public observer to traverse the list, and to observe up to $X$ on each of the elements. This restriction is visible in the signature of the head method, which returns a value of type String $\triangleleft X$.

Then, with polymorphic declassification we can implement data structures that are agnostic to the declassification policies of their elements, as well as polymorphic methods over these data structures. For example, we can construct declassificationpolymorphic lists of strings with the following cons method:

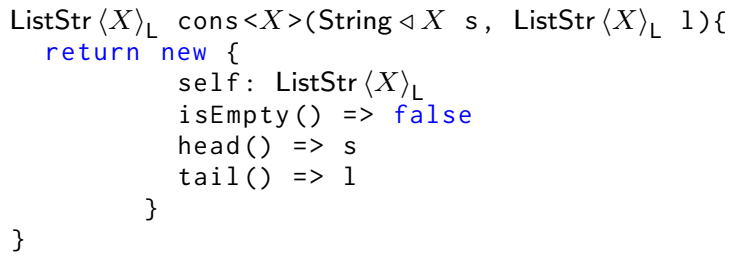

The cons method does not even access any method of list I, it simply returns a new declassification-polymorphic list of strings as a new object with the expected methods. We can then use this method to define a declassification-polymorphic list concatenation method concat: ListStr $\langle X\rangle_{\mathrm{L}} \times \operatorname{ListStr}\langle X\rangle_{\mathrm{L}} \rightarrow$ ListStr $\langle X\rangle_{\mathrm{L}}$ defined below:

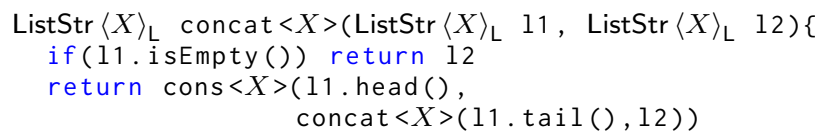

The concat and cons methods are standard object-oriented implementations of list concatenation and construction, respectively. The concat method respects the declassification type ListStr $\langle X\rangle$ of both lists because it uses I1.isEmpty () and I1.tail() to iterate over I1, and it uses $\mid 1$.head () to create a new declassification-polymorphic list of type ListStr $\langle X\rangle_{L}$. In particular, it uses no string-specific methods.

\section{B. Bounded Polymorphic Declassification}

The declassification interface ListStr $\langle X\rangle$ above is fully polymorphic, in that a public observer cannot exploit a priori any information about the elements of the list. In particular, it is not possible to implement a polymorphic contains method that would yield publicly-observable results. Indeed, contains needs to invoke eq over the elements of the list (obtained with head). Because the result of head has declassification type $X$, for any $X$, the results of equality comparisons are necessarily private.

In order to support polymorphic declassification more flexibly, we turn to bounded parametric polymorphism. Bounded parametric polymorphism supports the specification of both upper and lower bounds on type variables. The type ListStr $\langle X\rangle$ is therefore equivalent to ListStr $\langle X$ : String.. T $\rangle$, where the notation $X: A . . B$ is used to denote that $X$ is type variable that ranges between $A$ and $B$ ). Note that for ListStr to be well-formed, the declassification type variable $X$ must at least be a supertype of the safety type String.

Going back to declassification-polymorphic lists, if we want to allow the definition of methods like contains, we can further constrain the type variable $X$ to be a subtype of StringEq:

$$
\begin{aligned}
& \text { ListEqStr }\langle X \text { : String..StringEq }\rangle \triangleq \\
& \quad\left[\cdots, \text { tail : Unit }{ }_{\mathrm{L}} \rightarrow \text { ListEqStr }\langle X\rangle_{\mathrm{L}}\right]
\end{aligned}
$$

The type ListEqStr denotes a recursive polymorphic declassification policy that allows a public observer to traverse the list and compare its elements for equality with a given public element. With this policy we can implement a generic contains function with publicly-observable result:

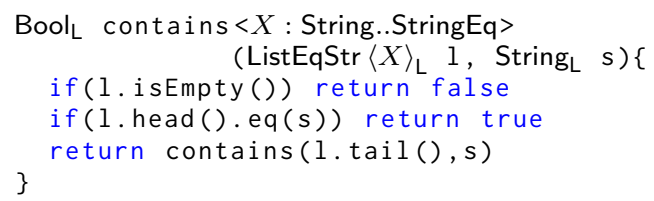

The key here is that I.head().eq(s) is guaranteed to be publicly observable, because the actual declassification policy with which $X$ will be instantiated necessarily includes (at least) the eq method. Thus, upper bounds on declassification variables are useful for supporting polymorphic clients.

As mentioned above, the lower bound of a type variable used for declassification must at least be the safety type for well-formedness. More interestingly, the lower bound plays 
a critical (dual) role for implementors of declassificationpolymorphic functions. Consider a method with signature

$$
\langle X: \text { String.. } \top\rangle \text { String } \triangleleft \text { StringLen } \rightarrow \text { String } \triangleleft X
$$

Can this method return non-public values? For instance, can it be the identity function? No, because returning a string of type String $\triangleleft$ StringLen would be unsound. Indeed, a client could instantiate $X$ with String, yielding

$$
\text { String } \triangleleft \text { StringLen } \rightarrow \text { String } \triangleleft \text { String }
$$

Therefore, to be sound for all possible instantiations of $X$, the implementor of the method has no choice but to return a public string.

To recover flexibility and allow a polymorphic implementation to return non-public values, we can constrain the lower bound of $X$. For instance

$$
\langle X \text { : StringLen.. } \top\rangle \text { String } \triangleleft \text { StringLen } \rightarrow \text { String } \triangleleft X
$$

admits the identity function as an implementation, in addition to other implementations that produce public results. Returned values cannot be more private than specified by the lower bound of $X$; their type must be a subtype of the lower bound.

Having illustrated the interest of upper and lower bounds of declassification type variables in isolation, we now present an example that combines both. Consider two lists of strings, each with one of the following declassification policies:

$$
\begin{aligned}
\text { ListStrLen }\langle X: & \text { String..StringLen }\rangle \triangleq \\
& {\left[\text { isEmpty : Unit } \rightarrow \text { Bool }_{\mathrm{L}},\right.} \\
& \text { head : Unit } \mathrm{L} \rightarrow \text { String } \triangleleft X, \\
& \text { tail : Unit } \left.\mathrm{L} \rightarrow \text { ListStrLen }_{\mathrm{L}}\right] \\
\text { ListStrFstLen } \triangleq & {\left[\text { isEmpty : Unit }{ }_{\mathrm{L}} \rightarrow \text { Bool }_{\mathrm{L}},\right.} \\
& \text { head : Unit } \mathrm{L}_{\mathrm{L}} \rightarrow \text { String } \triangleleft \text { StrFstLen, } \\
& \text { tail : Unit } \left.\mathrm{L}_{\mathrm{L}} \rightarrow \text { ListStrFstLen }_{\mathrm{L}}\right]
\end{aligned}
$$

ListStrLen is declassification polymorphic, ensuring that at least the length of its elements is declassified ( $X$ has upper bound StringLen). The second policy, ListStrFstLen, is monomorphic: it declassifies both the first character and the length of its elements. If we want a function able to concatenate these two string lists, its most general polymorphic signature ought to be:

$$
\begin{aligned}
& \langle X \text { : StrFstLen..StringLen }\rangle \\
& \quad \text { ListStrLen }\langle X\rangle_{\mathrm{L}} \times \text { ListStrFstLen }{ }_{\mathrm{L}} \rightarrow \text { ListStrLen }\langle X\rangle_{\mathrm{L}}
\end{aligned}
$$

The upper bound StringLen is required to have a valid instantiation of ListStrLen $\langle X\rangle$; the lower bound StrFstLen is required to be able to add elements of the second list to the returned list.

\section{Reasoning principles for PRNI}

Introducing polymorphism in declassification types yields an extended notion of type-based relaxed noninterference called polymorphic relaxed noninterference (PRNI). PRNI exactly characterizes that a program with polymorphic types must be secure for any instantiation of its type variables. To account for type variables, the judgment $\operatorname{PRNI}(\Delta, \Gamma, e, S)$ is parametrized by $\Delta$, a set of bounded type variables (i.e. $\Delta::=\cdot \mid \Delta, X: A . . B)$. As in $\operatorname{TRNI}(\Gamma, e, S)$, the closing typing environment $\Gamma$ specifies the secrecy of the inputs that $e$ can use, and $S$ specifies the observation level for the output. $\Delta$ gives meaning to the type variables that can occur in both $S$ and $\Gamma: e$ is secure for any instantiation of type variables that respects the bounds.

For instance, given $\Delta \triangleq X:$ StrFstLen..StringLen and $\Gamma \triangleq$ $x$ : String $\triangleleft X, \operatorname{PRNI}\left(\Delta, \Gamma\right.$, . length ()$\left., \operatorname{lnt} \mathrm{L}_{\mathrm{L}}\right)$ holds because for any type $T$ such as StrFstLen $<: T<$ : StringLen, and the knowledge that two input strings are related at String $\triangleleft T$, and hence at String $\triangleleft$ StringLen (i.e. both strings have the same length), the public observer does not learn anything new by executing $x$.length (). However, $\operatorname{PRNI}\left(\Delta, \Gamma\right.$, x.first () , String $\left.{ }_{\mathrm{L}}\right)$ does not hold. Note that if we substitute $X$ by StringLen, given two strings with the same length "abc" and "123", the public observer is able to distinguish them by executing "abc".first() and "123".first () and observing the results "a" and " 1 " as public values.

Also, $\operatorname{PRNI}(\Delta, \Gamma, x$, String $\triangleleft$ StringFst $)$ does not hold. Again, we can substitute $X$ by StringLen, and take input strings "abc" and "123", which can be discriminated by the public observer at type String $\triangleleft$ StringFst. However, $\operatorname{PRNI}(\Delta, \Gamma, x$, String $\triangleleft$ StringLen $)$ does hold: any two equivalent values at String $\triangleleft T$ where StrFstLen $<: T<$ : StringLen have at least the same length.

The rest of this paper dives into the formalization of polymorphic relaxed noninterference in a pure object-oriented setting (Sections $\mathrm{IV}$ and $\mathrm{V}$, , before discussing the necessary extensions to accommodate primitive types (Section VI).

\section{Formal SEMANTics}

We model polymorphic type-based declassification in $\mathrm{Ob}_{\mathrm{SEC}}^{\langle\rangle}$, an extension of the language $\mathrm{Ob}_{\mathrm{SEC}}$ [5] with polymorphic declassification. Ob $\mathrm{b}_{\mathrm{SEC}}$ is based on the object calculi of Abadi and Cardelli [8], and our treatment of type variables and bounded polymorphism is inspired by Featherweight Java [9] and DOT [10].

\section{A. Syntax}

Figure 2 presents the syntax of $\mathrm{Ob}_{\mathrm{SEC}}^{\langle\rangle}$. We highlight the extension for polymorphic declassification, compared to the syntax of ObSEC.

The language has three kind of expressions: objects, method invocations, variables. Objects $[z: S \Rightarrow \overline{m(x) e}]$ are collections of method definitions. Recall that the self variable $z$ binds the current object.

A security type is a faceted type $T \triangleleft U$, where $T$ is called the safety type of $S$, and $U$ is called the declassification type of $S$. Types $T$ include object types $O$ and self type variables $\alpha$. Declassification types $U$ additionally feature type variables $X$, to express polymorphic declassification. We use metavariables $A$ and $B$ for declassification type bounds. 


$$
\begin{aligned}
& e::=v|e \cdot m\langle U\rangle(e)| x \text { (terms) } \\
& v::=o \quad \text { (values) } \\
& o::=[z: S \Rightarrow \overline{m(x) e}] \quad \text { (objects) } \\
& S::=T \triangleleft U \quad \text { (security types) } \\
& T::=O \mid \alpha \quad \text { (types) } \\
& U, A, B::=T \mid X \quad \text { (declassification types) } \\
& O::=\operatorname{Obj}(\alpha) \cdot R \quad \text { (object types) } \\
& R::=[\overline{m: M}] \quad \text { (record types) } \\
& M::=\langle X: A . . B\rangle S \rightarrow S \text { (method signatures) } \\
& \Gamma::=\bullet \mid \Gamma, x: S \quad \text { (type environments) } \\
& \Phi::=\bullet \mid \Phi, \alpha<: \beta \quad \text { (subtyping environments) } \\
& \Delta::=\bullet \mid \Delta, X: A . . B \quad \text { (type } \\
& \alpha, \beta \quad \text { (self type variables) }
\end{aligned}
$$$$
\text { Fig. 2. } \mathrm{Ob}_{\mathrm{SEC}}^{\langle\rangle} \text {: Syntax }
$$

An object type $\operatorname{Obj}(\alpha) \cdot[m: M]$ is a collection of method signatures with unique names (we sometimes use $R$ to refer to just a collection of methods). The self type variable $\alpha$ binds to the defined object type (i.e. object types are recursive types). A method signature $\langle X: A . . B\rangle S_{1} \rightarrow S_{2}$ introduces the type variable $X$ with lower bound $A$ and upper bound $B$. To simplify the presentation of the calculus, we model singleargument methods with a single type variable ${ }^{2}$

\section{B. Subtyping}

Figure 3 presents the $\mathrm{Ob}_{\mathrm{SEC}}^{\langle\rangle}$subtyping judgment $\Delta ; \Phi \vdash$ $U_{1}<: U_{2}$. The type variable environment $\Delta$ is a set of type variables with their bounds, i.e. $\Delta::=\bullet \mid \Delta, X: A$..B. The subtyping environment $\Phi$ is a set of subtyping assumptions between self type variables, i.e. $\Phi::=\bullet \mid \Phi, \alpha<: \beta$

The rules for the monomorphic part of the language are similar to ObSEC. Rule (SObj) justifies subtyping between two object types; it holds if the methods of the left object type $\mathrm{O}_{1}$ are subtypes of the corresponding methods on $\mathrm{O}_{2}$. Both width and depth subtyping are supported. Note that to verify subtyping of method collections, i.e. $\Delta ; \Phi, \alpha<: \beta \vdash R_{1}<$ : $R_{2}$, we put in subtyping relation in $\Phi$ the self variables $\alpha$ and $\beta$. Rule (SVar) accounts for subtyping between self type variables and it holds if such subtyping relation exists in the subtyping environment.

The rules (STrans) and (SSubEq) justify subtyping by transitivity and type equivalence respectively. We consider type equivalence up to renaming and folding/unfolding of self type variables [5].

The novel part of $\mathrm{Ob}_{\mathrm{SEC}}^{\langle\rangle}$are type variables, handled by rules (SGVar1) and (SGVar2). We follow the approach of Rompf and Amin [10]. Rule (SGVar1) justifies subtyping between a type variable $X$ and a type $B$, if $B$ is the upper bound of

\footnotetext{
${ }^{2}$ The implementation supports both multiple arguments and multiple type variables.
}

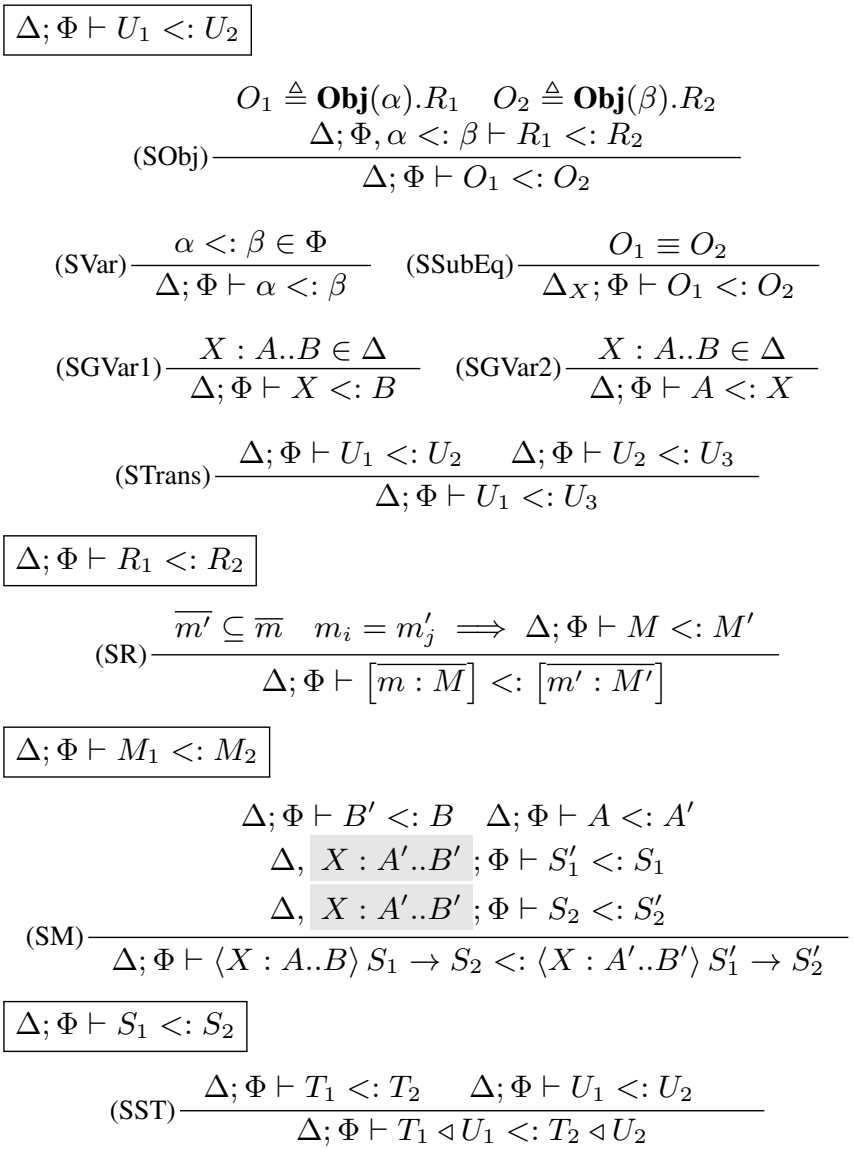

Fig. 3. $\mathrm{Ob}_{\mathrm{SEC}}^{\langle\rangle}$: Subtyping rules

the type variable in $\Delta$. Rule (SGVar2) is dual to (SGVar1), justifying that $A<: X$ if $A$ is the lower bound of $X$.

The judgment $\Delta ; \Phi \vdash R_{1}<: R_{2}$ accounts for subtyping between collections of methods, and is used in rule (SObj). The judgment $\Delta ; \Phi \vdash M_{1}<: M_{2}$ denotes subtyping between method signatures. For this judgment to hold, the type variable bounds $A^{\prime} . . B^{\prime}$ of the supertype (on the right) must be included within the bounds $A . . B$ of the subtype (on the left); this ensures that any instantiation on the right is valid on the left. Then, in a type variable environment extended with $X$ : $A^{\prime} . . B^{\prime}$, standard function subtyping must hold (contravariant on the argument type, covariant on the return type).

Finally, rule (SST) accounts for subtyping between security types, which requires facets to be pointwise subtypes.

\section{Type System}

The typing rules of $\mathrm{Ob}_{S E C}^{\langle\rangle}$appeal to some auxiliary definitions, given in Figure 4. Function $\operatorname{ub}(\Delta, U)$ returns the upper bound of a type $U$ in the type variable environment $\Delta$. Since $\mathrm{Ob}_{\mathrm{SEC}}^{\langle\rangle}$has a top type $(\mathbf{O b j}(\alpha)$. []) this recursive definition of ub is well-founded; as in Featherweight Java [9], we assume that $\Delta$ does not contain cycles. The auxiliary judgment $\Delta \vdash m \in U$ holds if method $m$ belongs to type $U$. For a type variable, this means that the method is in the 
$\mathrm{ub}(\Delta, U)=T$

$$
\frac{T \neq X}{\mathrm{ub}(\Delta, T)=T} \quad \frac{X: A . . B \in \Delta}{\mathrm{ub}(\Delta, X)=\mathrm{ub}(\Delta, B)}
$$

$\Delta \vdash m \in U$

$$
\frac{O \triangleq \mathbf{O b j}(\alpha) \cdot[\overline{m: M}]}{\Delta \vdash m_{i} \in O} \quad \frac{\Delta \vdash m \in \mathrm{ub}(\Delta, X)}{\Delta \vdash m \in X}
$$

$\operatorname{msig}(\Delta, U, m)=M$

$$
\begin{gathered}
\operatorname{msig}(\Delta, X, m)=\operatorname{msig}\left(\_, \mathrm{ub}(\Delta, X), m\right) \\
\frac{O \triangleq \mathbf{O b j}(\alpha) \cdot[\overline{m: M}]}{\operatorname{msig}\left({ }_{-}, O, m_{i}\right)=M[O / \alpha]}
\end{gathered}
$$

$\Delta \vdash U \in A . . B$

$$
\frac{\Delta ; \bullet \vdash A<: U \quad \Delta ; \bullet \vdash U<: B}{\Delta \vdash U \in A . . B}
$$

Fig. 4. $O \mathrm{Ob}_{\mathrm{SEC}}^{\langle\rangle}$: Some auxiliary definitions

upper bound $\mathrm{ub}(\Delta, X)$. Function $\operatorname{msig}(\Delta, U, m)$ returns the polymorphic method signature of method $m$ in type $U$. The rule for type variables looks up the signature in the upper bound. The rule for object types is standard; remark that it returns closed type signatures with respect to the self type variable. Finally, the judgment $\Delta \vdash U \in A . . B$ holds if the type $U$ is a super type of $A$ and a subtype of $B$ in the type variable environment $\Delta$.

Figure 5 presents the typing judgment $\Delta ; \Gamma \vdash e: S$ for $\mathrm{Ob}_{\mathrm{SEC}}^{\langle\rangle}$, which denotes that "expression $e$ has type $S$ under type variable environment $\Delta$ and type environment $\Gamma$ ". Note that in our presentation, we omit well-formedness rules for types and environments. They are included in Appendix A-E.

The first three typing rules are standard: rule (TVar) types a variable according to the environment, rule (TSub) is the subsumption rule and rule (TObj) types an object. The method definitions of the object must be well-typed with respect to the method signatures taken from the safety type $T$ of the security type $S$ ascribed to the self variable $z$. For this, the method body $e_{i}$ must be well-typed in an extended type variable environment with the type variable $\Delta, X: A_{i} . . B_{i}$, and an extended type environment with the self variable and the method argument.

Rules $(\mathrm{TmD})$ and $(\mathrm{TmH})$ cover method invocation, and account for declassification. The actual argument type $U^{\prime}$ must satisfy the variable bounds $\Delta \vdash U^{\prime} \in A$.. B. On the one hand, rule (TmD) applies when the method $m$ is in $U$ with signature $\langle X: A . . B\rangle S_{1} \rightarrow S_{2}$; this corresponds to a use of the object at its declassification interface. Then, the method invocation has type $S_{2}$ substituting $U^{\prime}$ for $X$. On the other hand, rule (TmH) applies when $m$ is not in $U$, but it is in $T$; this corresponds to a use beyond declassification and should raise the security

$$
\begin{aligned}
& \Delta ; \Gamma \vdash e: S \\
& (\mathrm{TVar}) \frac{x \in \operatorname{dom}(\Gamma)}{\Delta ; \Gamma \vdash x: \Gamma(x)}(\mathrm{TSub}) \frac{\Delta ; \Gamma \vdash e: S^{\prime} \quad \Delta ; \bullet \vdash S^{\prime}<: S}{\Delta ; \Gamma \vdash e: S} \\
& S \triangleq T \triangleleft U \quad \operatorname{msig}\left({ }_{-}, T, m_{i}\right)=\left\langle X: A_{i} . . B_{i}\right\rangle S_{i}^{\prime} \rightarrow S_{i}^{\prime \prime} \\
& \text { (TObj) } \frac{\Delta, X: A_{i} . . B_{i} ; \Gamma, z: S, x: S_{i}^{\prime} \vdash e_{i}: S_{i}^{\prime \prime}}{\Delta ; \Gamma \vdash[z: S \Rightarrow \overline{m(x) e}]: S} \\
& \Delta ; \Gamma \vdash e_{1}: T \triangleleft U \quad \Delta \vdash m \in U \\
& \operatorname{msig}(\Delta ; U, m)=\langle X: A . . B\rangle S_{1} \rightarrow S_{2} \\
& (\mathrm{TmD}) \frac{\Delta \vdash U^{\prime} \in A . . B \quad \Delta ; \Gamma \vdash e_{2}: S_{1}\left[U^{\prime} / X\right]}{\Delta ; \Gamma \vdash e_{1} . m\left\langle U^{\prime}\right\rangle\left(e_{2}\right): S_{2}\left[U^{\prime} / X\right]} \\
& \Delta ; \Gamma \vdash e_{1}: T \triangleleft U \quad \Delta \vdash m \notin U \\
& \operatorname{msig}(\Delta ; T, m)=\langle X: A . . B\rangle S_{1} \rightarrow T_{2} \triangleleft U_{2} \\
& (\mathrm{TmH}) \frac{\Delta \vdash U^{\prime} \in A . . B \quad \Delta ; \Gamma \vdash e_{2}: S_{1}\left[U^{\prime} / X\right]}{\Delta ; \Gamma \vdash e_{1} . m\left\langle U^{\prime}\right\rangle\left(e_{2}\right): T_{2}\left[U^{\prime} / X\right] \triangleleft \top}
\end{aligned}
$$

Fig. 5. $\mathrm{Ob}_{\mathrm{SEC}}^{\langle\rangle}$: Static semantics

methimpl $(o, m)=x . e$

$$
\begin{gathered}
\frac{o \triangleq[z: S \Rightarrow \overline{m(x) e}]}{\operatorname{methimpl}\left(o, m_{i}\right)=x \cdot e_{i}} \\
E::=[]|E \cdot m(e)| v \cdot m(E) \text { (evaluation contexts) } \\
\left(\text { EMInvO) } \frac{o \triangleq\left[z:_{-} \Rightarrow_{-}\right] \quad \text { methimpl }(o, m)=x . e}{E\left[o . m\left\langle \_\right\rangle(v)\right] \longmapsto E[e[o / z][v / x]]}\right.
\end{gathered}
$$

Fig. 6. $\mathrm{Ob}_{\mathrm{SEC}}^{\langle\rangle}$: Dynamic semantics

to high. This is why the result type is $T_{2}\left[X / U^{\prime}\right] \triangleleft \top$. This is all similar to the non-polymorphic rules (Figure 1), save for the type bounds check, and the type-level substitution.

\section{Dynamic Semantics}

The small-step dynamic semantics of $\mathrm{Ob}_{\mathrm{SEC}}^{\langle\rangle}$are standard, given in Figure 6 They rely on evaluation contexts and use the auxiliary function methimpl $(o . m)$ to lookup a method implementation. Note that types in general, and type variables in particular, do not play any role at runtime.

\section{E. Safety}

We first define what it means for a closed expression $e$ to be safe: an expression is safe if it evaluates to a value, or diverges without getting stuck.

Definition 1 (Safety). $\operatorname{safe}(e) \Longleftrightarrow \forall e^{\prime} . e \longmapsto{ }^{*} e^{\prime} \Longrightarrow e^{\prime}=$ $v$ or $\exists e^{\prime \prime} . e^{\prime} \longmapsto e^{\prime \prime}$

Well-typed $\mathrm{Ob}_{\mathrm{SEC}}^{\langle\rangle}$closed terms are safe.

Theorem 1 (Syntactic type safety). $\vdash e: S \Longrightarrow \operatorname{safe}(e)$

But of course, type safety is far from sufficient; we want to make sure that well-typed $\mathrm{Ob}_{\mathrm{SEC}}^{\langle\rangle}$terms are secure. To this 
end, the next section formalizes the precise notion of security we consider in $\mathrm{Ob}_{\mathrm{SEC}}^{\langle\rangle}$, and proves that it is implied by typing.

\section{Polymorphic Relaxed Noninterference, FORMALLY}

We now formally define the security property of polymorphic type-based relaxed noninterference (PRNI), and prove that the $\mathrm{Ob}_{\mathrm{SEC}}^{\langle\rangle}$type system soundly enforces PRNI.

\section{A. Logical Relation}

We define how values, terms and environments are related through a step-indexed logical relation [11] (Figure 77. Stepindexing is needed to ensure that the logical relation is wellfounded in presence of recursive object types.

The main novelty of this logical relation with respect that of $\mathrm{Ob}_{S E C}$ is that it needs to give an interpretation to polymorphic security types of the form $T \triangleleft X$. We do this by quantifying over all possible actual types $U$ for $X$ and interpreting $T \triangleleft U$. The interpretation of a security type is expressed as sets of atoms of the form $\left(k, e_{1}, e_{2}\right)$, where $k$ is a step index meaning that $e_{1}$ and $e_{2}$ are related for $k$ steps.

The definition also appeals to a simple typing judgment $\Gamma \vdash_{1} e: T$, which disregards the declassification types, and is therefore standard (Appendix A-J). We have that $\Delta ; \Gamma \vdash e$ : $T \triangleleft U \Rightarrow \Gamma \vdash_{1} e: T$. The use of this simple type system in the logical relation clearly separates the definitions of security from its static enforcement by the type system of \$IV-C [5].

The logical relation uses several auxiliary definitions. Atom $_{n}[T]$ requires $e_{1}$ and $e_{2}$ to be simply well-typed expressions of type $T$ and the index $k$ to be strictly less than $n$. Atom $_{n}^{v a l}[T]$ restricts Atom ${ }_{n}[T]$ to values. Atom $[T]$ are atoms of simply well-typed expressions of type $T$ (i.e. for any stepindex $k$ ).

The definition of $\mathcal{V} \llbracket T \triangleleft O \rrbracket$ relates two objects $o_{1}, o_{2}$ for $k$ steps if for any method $m \in O$ and with signature $\langle X: A . . B\rangle S^{\prime} \rightarrow S^{\prime \prime}$ and $j<k$, given related arguments for $j$ steps at $S^{\prime}$, invocations of $m$ produce related results for $j$ steps at $S^{\prime \prime}$. More specifically, given any actual type $T^{\prime}$ that satisfies the bounds of the type parameter $X$ (i.e., $T^{\prime} \in A . . B$ ) and given related arguments in $\mathcal{V} \llbracket S^{\prime}\left[T^{\prime} / X\right] \rrbracket$ we must obtain related computations in $\mathcal{V} \llbracket S^{\prime \prime}\left[T^{\prime} / X\right] \rrbracket$.

The relational interpretation of expressions $\mathcal{C} \llbracket T \triangleleft U \rrbracket$ relates atoms of the form $\left(k, e_{1}, e_{2}\right)$ that satisfy that for all $j<k$, if both expressions $e_{1}$ and $e_{2}$ reduce to values $v_{1}$ and $v_{2}$ in at most $k$ steps then $v_{1}$ and $v_{2}$ must be equivalent for the remaining $k-j$ steps. This definition is terminationinsensitive: if one expression does not terminate in less that $k$ steps, then the two expressions are trivially equivalent.

Type environments have standard interpretations. $\mathcal{G} \llbracket \Gamma \rrbracket$ relates value substitutions $\gamma$, i.e. mappings from variables to closed values, as triples of the form $\left(k, \gamma_{1}, \gamma_{2}\right)$, where $\gamma_{1}$ and $\gamma_{2}$ are related if they have the same variables as $\Gamma$, and for any variable $x$, the associated values are related for $k$ steps at type $\Gamma(x)$. Finally, a type substitution $\sigma$, i.e. a mapping from type variables to closed types, satisfies a type variable environment
$\Delta$, noted $\mathcal{D} \llbracket \Delta \rrbracket$, if it has the same type variables that $\Delta$ and the mapped type $T$ is within the type variable bounds.

\section{B. Defining Polymorphic Relaxed Noninterference}

Having defined the logical relation, we can now formally define PRNI. As standard, noninterference properties allow modular reasoning about open terms with respect to (termlevel) variables. For PRNI, we extend this modular reasoning principle to open terms with respect to type variables. Then, a simply well-typed expression $e$ under $\Delta$ and a well-formed $\Gamma$ satisfies PRNI at well-formed type $S$, written $\operatorname{PRNI}(\Delta, \Gamma, e, S)$, if for any type substitution $\sigma$ that satisfies $\Delta$ and two value substitutions $\gamma_{1}$ and $\gamma_{2}$ in the relational interpretation of $\sigma(\Gamma)$, applying the two value substitutions to the expression $e$ produces equivalent expressions at type $\sigma(S)$. As usual, the definition quantifies universally on the step index $k$. We need only consider a single type substitution $\sigma$; indeed, type variables happen only in declassification types, which express the observation power of the public observer. Therefore, for each security type of the form $T \triangleleft X$ we only need to consider one actual type $U$ within the bounds of $X$ to pick the observation power of the public observer. The substitution $\sigma$ captures all these choices.

Definition 2 (Polymorphic relaxed noninterference).

$$
\begin{aligned}
& \operatorname{PRNI}(\Delta, \Gamma, e, S) \Longleftrightarrow \\
& \quad S \triangleq T \triangleleft U \quad \Gamma \vdash_{1} e: T \wedge \Delta \vdash \Gamma \wedge \Delta \vdash S \wedge \\
& \quad \forall k \geq 0 . \forall \sigma, \gamma_{1}, \gamma_{2} . \sigma \in \mathcal{D} \llbracket \Delta \rrbracket .\left(k, \gamma_{1}, \gamma_{2}\right) \in \mathcal{G} \llbracket \sigma(\Gamma) \rrbracket \\
& \quad \Longrightarrow\left(k, \sigma\left(\gamma_{1}(e)\right), \sigma\left(\gamma_{2}(e)\right)\right) \in \mathcal{C} \llbracket \sigma(S) \rrbracket
\end{aligned}
$$

This definition captures the intuitive security notion that an expression is secure if it produces indistinguishable outputs up to the declassification power of the public observer (specified by $S$ ), when linked with indistinguishable inputs up to their declassification (specified by $\Gamma$ ).

\section{Security Type Soundness}

To establish that all well-typed $\mathrm{Ob}_{\mathrm{SEC}}^{\langle\rangle}$terms satisfy PRNI, we first introduce a notion of logically-related open terms, and prove that if an expression is related to itself, then it satisfies PRNI. We then prove the fundamental property of the logical relation, which states that well-typed terms are logically-related to themselves.

Two open expressions $e_{1}$ and $e_{2}$ are logically related at type $S$ in environments $\Delta$ and $\Gamma$ if, given a type substitution $\sigma$ satisfying $\Delta$ and value substitutions $\gamma_{1}$ and $\gamma_{2}$ in the relational interpretation of $\sigma(\Gamma)$, closing these expressions with the given substitutions produces related expressions related at type $\sigma(S)$.

Definition 3 (Logical relatedness of open terms).

$$
\begin{aligned}
& \Delta ; \Gamma \vdash e_{1} \approx e_{2}: S \Longleftrightarrow \\
& \Delta ; \Gamma \vdash e_{i}: S \wedge \Delta \vdash \Gamma \wedge \Delta \vdash S \wedge \\
& \forall k \geq 0 . \forall \sigma, \gamma_{1}, \gamma_{2} . \sigma \in \mathcal{D} \llbracket \Delta \rrbracket . \\
& \quad\left(k, \gamma_{1}, \gamma_{2}\right) \in \mathcal{G} \llbracket \sigma(\Gamma) \rrbracket \\
& \quad \Longrightarrow\left(k, \sigma\left(\gamma_{1}\left(e_{1}\right)\right), \sigma\left(\gamma_{2}\left(e_{2}\right)\right) \in \mathcal{C} \llbracket \sigma(S) \rrbracket\right.
\end{aligned}
$$




$$
\begin{aligned}
& \operatorname{Atom}_{n}[T] \quad=\left\{\left(k, e_{1}, e_{2}\right) \mid k<n \wedge \vdash_{1} e_{1}: T \wedge \vdash_{1} e_{2}: T\right\} \\
& \operatorname{Atom}_{n}^{\text {val }}[T] \quad=\left\{\left(k, v_{1}, v_{2}\right) \in \operatorname{Atom}_{n}[T]\right\} \\
& \text { Atom }[T] \quad=\left\{\left(k, e_{1}, e_{2}\right) \in \bigcup_{n \geq 0} \operatorname{Atom}_{n}[T]\right\} \\
& \mathcal{V} \llbracket T \triangleleft O \rrbracket \quad=\left\{\left(k, v_{1}, v_{2}\right) \in \operatorname{Atom}[T] \mid\right. \\
& \left(\forall m \in O . \quad \operatorname{msig}(O, m)=\langle X: A . . B\rangle S^{\prime} \rightarrow S^{\prime \prime}\right. \\
& \forall j<k, T^{\prime}, v_{1}^{\prime}, v_{2}^{\prime} . \quad \vdash T^{\prime} \wedge T^{\prime} \in A . . B \wedge \\
& \left(j, v_{1}, v_{2}\right) \in \mathcal{V} \llbracket T \triangleleft O \rrbracket \wedge\left(j, v_{1}^{\prime}, v_{2}^{\prime}\right) \in \mathcal{V} \llbracket S^{\prime}\left[T^{\prime} / X\right] \rrbracket \Longrightarrow \\
& \left.\left(j, v_{1} . m\left\langle\left\langle_{-}\right\rangle\left(v_{1}^{\prime}\right), v_{2} . m\left\langle \_\right\rangle\left(v_{2}^{\prime}\right)\right) \in \mathcal{C} \llbracket S^{\prime \prime}\left[T^{\prime} / X\right] \rrbracket\right)\right\} \\
& \mathcal{C} \llbracket T \triangleleft U \rrbracket \quad=\left\{\left(k, e_{1}, e_{2}\right) \in \operatorname{Atom}[T] \mid\left(\forall j<k .\left(e_{1} \longmapsto \leq j v_{1} \wedge e_{2} \longmapsto \leq j v_{2}\right) \Longrightarrow\left(k-j, v_{1}, v_{2}\right) \in \mathcal{V} \llbracket T \triangleleft U \rrbracket\right)\right\} \\
& \mathcal{G} \llbracket \rrbracket \quad=\{(k, \emptyset, \emptyset)\} \\
& \mathcal{G} \llbracket \Gamma ; x: S \rrbracket \quad=\left\{\left(k, \gamma_{1}\left[x \mapsto v_{1}\right], \gamma_{2}\left[x \mapsto v_{2}\right]\right) \mid\left(k, \gamma_{1}, \gamma_{2}\right) \in \mathcal{G} \llbracket \Gamma \rrbracket \wedge\left(k, v_{1}, v_{2}\right) \in \mathcal{V} \llbracket S \rrbracket\right\} \\
& \mathcal{D} \llbracket \rrbracket \quad=\{\emptyset\} \\
& \mathcal{D} \llbracket \Delta ; X: A . . B \rrbracket=\{\sigma[X \mapsto T] \mid \sigma \in \mathcal{D} \llbracket \Delta \rrbracket \wedge \Delta \vdash T \in A . . B\}
\end{aligned}
$$

Fig. 7. $\mathrm{Ob}_{\mathrm{SEC}}^{\langle\rangle}$Step-indexed logical relation for type-based equivalence

Trivially, if an expression is logically related to itself, then it satisfies PRNI.

Lemma 2 (Self logical relation implies PRNI). $\Delta ; \Gamma \vdash e \approx e: S \Longrightarrow \operatorname{PRNI}(\Delta, \Gamma, e, S)$

We then turn to proving that all well-typed terms are logically-related to themselves, i.e. the fundamental property of the logical relation.

Theorem 3 (Fundamental property).

$\Delta ; \Gamma \vdash e: S \Longrightarrow \Delta ; \Gamma \vdash e \approx e: S$

Proof. The proof is by induction on the typing derivation of $e$. We use the common approach of defining compatibility lemmas for each typing rule [11]. Each case follows from the corresponding compatibility lemma.

Security type soundness follows directly from Theorem 3 and Lemma 2 .

Theorem 4 (Security type soundness).

$\Delta ; \Gamma \vdash e: S \Longrightarrow \operatorname{PRNI}(\Delta, \Gamma, e, S)$

Having proven that well-typed $\mathrm{Ob}_{\mathrm{SEC}}^{\langle\rangle}$programs are secure, we are almost ready to revisit the examples of Section III to illustrate PRNI. We must first address the case of primitive types, discussed next.

\section{AD-HOC POLYMORPHISM FOR PRIMITIVE TYPES}

Both $\mathrm{Ob}_{\mathrm{SEC}}$ [5] and $\mathrm{Ob}_{\mathrm{SEC}}^{\langle\rangle}$(so far) ignore the case of primitive types, such as integers and strings. However, in an object-oriented language, primitive types are both necessary and challenging from a security viewpoint. In particular, integrating type-based declassification with faceted types requires appealing to a form of ad hoc polymorphism.

\section{A. The Need and Challenge of Primitive Types}

In a pure object-oriented calculus (as in a pure functional calculus) without primitive types, the only real observation that can be made on programs is termination. A terminationinsensitive notion of noninterference is therefore useless in a pure setting: one needs some primitive types with a purely syntactic notion of equality. Indeed, all the examples we presented in earlier sections assume a syntactic notion of observation for strings and integers.

Introducing primitive types calls for some form of label polymorphism. Indeed, we do not want to fix the security level of primitive operations, as this would be either impractical for the public observer (if all security labels were high) or for the secret observer (if all security labels were low). This is why practical security-typed languages like FlowCaml [7] and Jif [6] use label-polymorphic primitive operators, specifying that the return label is the least upper bound of the argument labels. For instance, a binary integer operator would have type $\forall \ell_{1}, \ell_{2} . \operatorname{lnt} \ell_{\ell_{1}} \times \operatorname{Int}_{\ell_{2}} \rightarrow \operatorname{Int}_{\ell_{1} \sqcup \ell_{2}}$. In a monomorphic security language, the same principle is hardcoded in the typing rules for primitive operators [12].

Unfortunately this approach does not work with labels-astypes, even in a label-monomorphic setting. Indeed, because labels are types, returning the join of the argument security labels means computing the subtyping join (denoted $\sqcup_{<:}$hereafter) of the declassification types. This is both impractical, incorrect, and potentially unsound from a security viewpoint:

- Impractical. Consider a function of type Bool $\triangleleft X_{1} \times$ Int $\triangleleft X_{2} \rightarrow$ Bool $\triangleleft\left(X_{1} \sqcup_{<:} X_{2}\right)$. Given two public arguments (i.e. $X_{1}=$ Bool, $X_{2}=\operatorname{lnt}$ ), then assuming Bool $\sqcup_{<:}$Int $=\top$, the result is necessarily private. While sound, this is way too conservative; it is impractical for primitive operations to always return private values even when given public inputs.

- Incorrect. Consider an integer comparison of type Int $\triangleleft X_{1} \times$ Int $\triangleleft X_{2} \rightarrow$ Bool $\triangleleft\left(X_{1} \sqcup_{<:} X_{2}\right)$. If we instantiate this signature with Int and Int we obtain an illformed return type, Bool $\triangleleft$ Int.

- Insecure. Consider a unary integer operator Int $\triangleleft X \rightarrow$ Int $\triangleleft X$; this signature is not sound security-wise for all 
unary integer operators. Take an operator that trims the most-significant bit of its argument. If one declassifies only the parity of the argument, two equivalent inputs will not always yield two equivalent outputs (as the parity of the trimmed values might differ).

\section{B. Sound Signatures for Primitive Types}

The observations above reveal one of the flip sides of expressive declassification policies: because declassification is captured semantically, declassification polymorphism is a very strong notion compared to standard label polymorphism. In the general case, without appealing to intricate semantic conditions, there are therefore only two simple syntactic principles to define sound signatures for primitive operators $3^{3}$

(P1) if every argument is public (e.g. String $\left.\mathrm{L}_{\mathrm{L}}\right)$ then the return type can be public.

(P2) if any argument is not public (e.g. String $\triangleleft$ StringFst) then the return type must be secret (e.g. String ${ }_{H}$ ).

As typical, we provide an object-oriented interface for primitive types (e.g. $a+b$ is $a .+(b)$ as in Scala for instance). Therefore the principles above must be extended to account for the status of the receiver object: if the primitive method invoked on the primitive value is part of its declassification type, then it is considered a public "argument"; otherwise, it is private.

Note that, without any form of polymorphism, i.e. picking a single syntactic principle above, primitive types would be impractical. Duplicating all definitions to offer both options is also not a viable approach.

\section{Polymorphic Primitive Signatures}

To support the two syntactic principles exposed above, we propose to use ad-hoc polymorphism (akin to overloading) for primitive types $P$ in $\mathrm{Ob}_{\mathrm{SEC}}^{\langle\rangle}$. We introduce polymorphic primitive signatures, written $P \triangleleft * \rightarrow P \triangleleft *$. A primitive security type $P \triangleleft *$ is resolved polymorphically at use site, following principles (P1) and (P2) above. Object-oriented interfaces for primitive types are exclusively composed of polymorphic primitive signatures. For instance, in $\mathrm{Ob}_{S E C}^{\langle\rangle}$ strings are primitives, declared by the following String type:

$$
\begin{aligned}
\text { String } \triangleq & {[\text { concat : String } \triangleleft * \rightarrow \text { String } \triangleleft *,} \\
& \text { first : Unit } \triangleleft * \rightarrow \text { String } \triangleleft *, \\
& \text { length : Unit } \triangleleft * \rightarrow \operatorname{lnt} \triangleleft *, \\
& \text { eq : String } \triangleleft * \rightarrow \text { Bool } \triangleleft *, \\
& \cdots]
\end{aligned}
$$

To illustrate, assume a : String $, \mathrm{b}:$ String $\mathrm{L}_{\mathrm{L}}$ and $\mathrm{c}:$ String $_{\mathrm{H}}$. Then a.eq(b) has type Bool , while a.eq(c) has type Bool $l_{H}$.

Primitive types can also be subject to declassification policies. For instance, consider:

$$
\text { StringEqPoly } \triangleq[\text { eq : String } \triangleleft * \rightarrow \text { Bool } \triangleleft *]
$$

\footnotetext{
${ }^{3}$ The syntactic principles (P1) and (P2) are formally justified by the proof of Lemma 5 . discussed in Section 11
}

$$
\begin{aligned}
e::=\cdots \mid \text { e.m(e) } & \text { (terms) } \\
v::=\cdots \mid \mathrm{p} & \text { (values) } \\
T::=\cdots \mid P & \text { (types) } \\
M::=\cdots \mid I & \text { (method signatures) } \\
S::=\cdots \mid P \triangleleft * & \text { (security types) } \\
I::=P \triangleleft * \rightarrow P \triangleleft * & \text { (primitive signatures) } \\
P::=(\text { eg. Int, String) } & \text { (primitive types) } \\
\Phi::=\cdots \mid \Phi, P<: \beta & \text { (subtyping environments) }
\end{aligned}
$$

Fig. 8. $\mathrm{Ob}_{\mathrm{SEC}}^{\langle\rangle}$: Extended syntax for primitive types

$$
\begin{aligned}
& \Delta ; \Phi \vdash U_{1}<: U_{2} \\
& \begin{array}{r}
\operatorname{meths}(P)=R_{1} \quad O \triangleq \mathbf{O b j}(\beta) \cdot R_{2} \\
\Delta \quad \quad(\text { SPObj }) \frac{\Delta, P<: \beta \vdash R_{1}<: R_{2}}{\Delta ; \Phi \vdash P<: O}
\end{array} \\
& \text { (SPVar) } \frac{P<: \beta \in \Phi}{\Delta ; \Phi \vdash P<: \beta} \\
& \Delta ; \Phi \vdash M_{1}<: M_{2} \\
& \cdots \quad(\text { SImpl }) \frac{}{\Delta ; \Phi \vdash I<: I}
\end{aligned}
$$

Fig. 9. $\mathrm{Ob}_{\mathrm{SEC}}^{\langle\rangle}\langle$Subtyping rules for primitive types

and d : String $\triangleleft$ StringEqPoly. Then d.eq(b) has type Bool L, while d.concat(a) : String . $_{\text {. }}$.

Furthermore, one can use any type signature in a declassification policy for a primitive type, as long as it is sound. For instance, StringEqL $\triangleq[$ eq : String $L \rightarrow$ Bool $L$ ] respects principle 1). Conversely, StringEqBad $\triangleq\left[\right.$ eq : String ${ }_{H} \rightarrow$ Bool $\left._{L}\right]$ cannot be used as it would violate the soundness principles above (in $\mathrm{Ob}_{\mathrm{SEC}}^{\langle\rangle}$, String $\triangleleft$ StringEqBad is ill-formed).

\section{Formal Semantics}

We now formalize the treatment of primitive types in $\mathrm{Ob}_{S E C}^{\langle\rangle}$. Figure 8 presents the extended syntax to support primitive values $\mathrm{p}$ and primitive types $P$. Expression $\operatorname{e.m}(e)$ is for method invocation on primitives; as explained previously, primitive types expose an object-oriented interface, so $a+b$ is $a .+(b)$. We extend the category $S$ with security types of the form $P \triangleleft *$ and introduce a new category $I$, for primitive signatures $P \triangleleft * \rightarrow P \triangleleft *$. The security type $P \triangleleft *$ can be used for standard signatures $\langle X: A . . B\rangle S_{1} \rightarrow S_{2}$ as well.

The changes to subtyping are in Figure 9 Now, subtyping assumptions can be also made between a primitive type and a self type variable, i.e. $\Phi::=\bullet|\Phi, \alpha<: \beta| \Phi, P<: \beta$, and function meths returns the methods of a primitive type. Rule (SPObj) justifies subtyping between a primitive type and an object type, and it is very similar to rule (SObj) of Figure 3 for object types. Rule (SPVar) accounts for subtyping between primitive types and type variables and it holds if such subtyping relation exists in the subtyping environment $\Phi$. Note that there is no rule for subtyping between an object type 
$\Delta ; \Gamma \vdash e: S$

$$
\begin{array}{cc}
\cdots \quad(\text { TPrim }) \frac{P=\Theta(\mathrm{p})}{\Delta ; \Gamma \vdash \mathrm{p}: P \triangleleft P} \\
\Delta ; \Gamma \vdash e_{1}: T \triangleleft U \quad \Delta \vdash m \in U \\
\operatorname{msig}(\Delta, U, m)=P_{1} \triangleleft * \rightarrow P_{2} \triangleleft * \\
\Delta ; \Gamma \vdash e_{2}: P_{1} \triangleleft U_{1} \\
(\mathrm{TPmD}) \frac{\operatorname{rdecl}\left(P_{1} \triangleleft U_{1}, P_{2}\right)=P_{2}^{\prime}}{\Delta ; \Gamma \vdash e_{1} \cdot m\left(e_{2}\right): P_{2} \triangleleft P_{2}^{\prime}} \\
\begin{array}{c}
\Delta ; \Gamma \vdash e_{1}: T \triangleleft U \quad \Delta \vdash m \notin U \\
\operatorname{msig}(\Delta, T, m)=P_{1} \triangleleft * \rightarrow P_{2} \triangleleft * \\
\Delta ; \Gamma \vdash e_{2}: P_{1} \triangleleft U_{1}
\end{array} \\
(\mathrm{TPmH}) \frac{\Delta ; \Gamma \vdash e_{1} . m\left(e_{2}\right): P_{2} \triangleleft \top}{}
\end{array}
$$

$\operatorname{rdecl}\left(P_{1} \triangleleft U_{1}, P_{1}\right)=U$

$$
\operatorname{rdecl}\left(P_{1} \triangleleft U_{1}, P_{2}\right)= \begin{cases}P_{2} & P_{1}=U_{1} \\ \top & \text { otherwise }\end{cases}
$$

Fig. 10. $\mathrm{Ob}_{\mathrm{SEC}}^{\langle\rangle}$: Extended static semantics for primitive types

and a primitive type, because this would not be sound. Rule (SImpl) accounts for subtyping between the same primitive signature. There is no rule to justify subtyping between a primitive signature and a standard signature.

As we discussed at the end of Section VI-B, we need an extra condition for the well-formedness of security types to ensure sound declassification. Given the type $T \triangleleft U$, if $T$ has a method $m: I$, the method signature of $m$ in $U$ must be either the same primitive signature $I$, or a normal signature that is sound. We use the predicate soundsig to express that signature $\langle X: A . . B\rangle S_{1} \rightarrow S_{2}$ is sound, which must satisfy that either the argument type is public, or the return type is private:

$$
\text { soundsig }\left(\left\langle_{-}\right\rangle P \triangleleft U_{1} \rightarrow T_{2} \triangleleft U_{2}\right) \Longleftrightarrow U_{1}=P \vee U_{2}=\top
$$

Figure 10 presents the extension to the typing rules of $\mathrm{Ob}_{\mathrm{SEC}}^{\langle\rangle}$. Rule (TPrim) justifies typing for primitive values, using a function $\Theta$ that specifies each primitive type. The new typing rules (TPmD) and (TPmH) realize ad hoc polymorphism for primitive types. Rule (TPmD) is key: it applies when $m$ is in the declassification type $U$, and uses the function rdecl to calculate the declassification type of the return type, based on the type of the argument: if the argument is public, so is the returned value. Rule (TPmH) applies when $m$ is not in the declassification type $U$, and similarly to (TmH), ensures that the returned value is private.

Figure 11 shows the extension to the dynamic semantics to support primitive values. Rule (EMInvP) executes a method invocation on a primitive value using the function $\theta$, which abstracts over the internal implementation of primitive values.

To prove type safety for $\mathrm{Ob}_{\mathrm{SEC}}^{\langle\rangle}$with primitives, we only need to assume that $\Theta$ and $\theta$-which are parameters of the language-agree on the specification and implementation of all primitive types and their operations.

$$
\left(\text { EMInvP) } \frac{}{E\left[\mathrm{p}_{1} \cdot m\left(\mathrm{p}_{2}\right)\right] \longmapsto E\left[\theta\left(m, \mathrm{p}_{1}, \mathrm{p}_{2}\right)\right]}\right.
$$

Fig. 11. $\mathrm{Ob}_{\mathrm{SEC}}^{\langle 〉}$ : Dynamic semantics of primitive values

$$
\begin{aligned}
\mathcal{V} \llbracket P \triangleleft P \rrbracket= & \{(k, \mathrm{p}, \mathrm{p}) \in \operatorname{Atom}[P]\} \\
\mathcal{V} \llbracket T \triangleleft O \rrbracket= & \left\{\left(k, v_{1}, v_{2}\right) \in \operatorname{Atom}[T] \mid \ldots\right. \\
& \forall m \in O . \operatorname{msig}(\cdot, O, m)=P_{1} \triangleleft * \rightarrow P_{2} \triangleleft * \\
& \forall j<k, v_{1}^{\prime}, v_{2}^{\prime} \cdot U_{1}>: P_{1} \\
& \left(\left(j, v_{1}^{\prime}, v_{2}^{\prime}\right) \in \mathcal{V} \llbracket P_{1} \triangleleft U_{1} \rrbracket \Longrightarrow\right. \\
& \left.\left.\left(j, v_{1} . m\left(v_{1}^{\prime}\right), v_{2} . m\left(v_{2}^{\prime}\right)\right) \in \mathcal{C} \llbracket P_{2} \triangleleft \operatorname{rdecl}\left(P_{1} \triangleleft U_{1}, P_{2}\right) \rrbracket\right)\right\}
\end{aligned}
$$

Fig. 12. $\mathrm{Ob}_{\mathrm{SEC}}^{\langle\rangle}$: Step-indexed logical relation with new definitions for primitive types

\section{E. Logical Relation for Primitive Types}

Figure 12 presents the extension to the logical relation of Figure 7 to account for primitive types. First, $\mathcal{V} \llbracket P \triangleleft P \rrbracket$ relates syntactically equal primitive values of type $P$. Second, the definition of $\mathcal{V} \llbracket T \triangleleft O \rrbracket$ now accounts for primitive values that are observed with a declassification type $O . \mathcal{V} \llbracket T \triangleleft O \rrbracket$ still relates values $v_{1}$ and $v_{2}$ if, for all methods of $O$, given related arguments, the invocations of $m$ on $v_{1}$ and $v_{2}$ produce related computations. However, the definition now discriminates between each type of signatures. For a method $m$ with primitive signature $P_{1} \triangleleft * \rightarrow P_{2} \triangleleft *$, we require one of the following conditions to hold. If we get related arguments $v_{1}^{\prime}$ and $v_{2}^{\prime}$ at $P_{1} \triangleleft P_{1}$ (i.e. public values), method invocations $v_{1} \cdot m\left(v_{1}^{\prime}\right)$ and $v_{2} \cdot m\left(v_{2}^{\prime}\right)$ need to be related at the public type $P_{2} \triangleleft P_{2}$. Otherwise, if the arguments $v_{1}^{\prime}$ and $v_{2}^{\prime}$ are related at a nonpublic type $\left(P_{1} \triangleleft U, P_{1} \neq U\right)$, then $v_{1} \cdot m\left(v_{1}^{\prime}\right)$ and $v_{2} \cdot m\left(v_{2}^{\prime}\right)$ need to be related at the top type $P \triangleleft \top$. These conditions are expressed in the definition by requiring related method invocations in $\mathcal{C} \llbracket P_{2} \triangleleft \operatorname{rdecl}\left(P_{1} \triangleleft U_{1}, P_{2}\right) \rrbracket$.

Extending the fundamental property (Lemma 3 ) for primitive types requires the following lemma, which states that syntactically-equal primitive values of type $P$ are in the objectoriented interpretation of any type $P \triangleleft O$ - essentially, equal values cannot be discriminated.

Lemma 5 (Equal values are logically related).

$\forall k \geq 0, \mathrm{p}, P, O$.

$$
\vdash_{1} \mathrm{p}: O \wedge P<: O \Longrightarrow(k, \mathrm{p}, \mathrm{p}) \in \mathcal{V} \llbracket P \triangleleft O \rrbracket
$$

Proof. Because $P \triangleleft O$ is a well-formed security type, $O$ consists of primitive signatures and standard, sound signatures. For the case of primitive signatures, at some point we have $P_{1} \triangleleft * \rightarrow P_{2} \triangleleft *$ and equivalent values at $\left(j, v_{1}, v_{2}\right) \in$ $P_{1} \triangleleft U_{1}$ and we have to prove that $\left(j, \delta\left(m, b, v_{1}\right), \delta\left(m, b, v_{2}\right) \in\right.$ $\mathcal{C} \llbracket P_{2} \triangleleft \operatorname{rdecl}\left(P_{1} \triangleleft U_{1}, P_{2}\right) \rrbracket$. We do case analysis on $U$. If $U_{1}=P_{1}$, we know that $v_{1}=v_{2}$ and hence if $\delta\left(m, b, v_{1}\right)$ and $\delta\left(m, b, v_{2}\right)$ are defined, their results are syntactically equal, so $\left.\left(j, \delta\left(m, b, v_{1}\right), \delta\left(m, b, v_{2}\right)\right) \in \mathcal{C} \llbracket P_{2} \triangleleft P_{2}\right) \rrbracket$. If $U \neq$ $P_{1}$, then the proof obligation is $\left(j, \delta\left(m, b, v_{1}\right), \delta\left(m, b, v_{2}\right) \in\right.$ $\mathcal{C} \llbracket P_{2} \triangleleft \top \rrbracket$; this is trivial because any two values are re- 
lated at $T$. For the case of standard signatures, at some point we have $P_{1} \triangleleft U_{1} \rightarrow P_{2} \triangleleft U_{2}$ and we have to prove that $\left(j, \delta\left(m, b, v_{1}\right), \delta\left(m, b, v_{2}\right)\right) \in \mathcal{C} \llbracket P_{2} \triangleleft \operatorname{rdecl}\left(P_{1} \triangleleft U_{1}, P_{2}\right) \rrbracket$. Since the signature is sound, we know that either $U_{1}=P_{1}$ or $U_{2}=\top$; then the result follows similarly to the primitive signature case.

Note that the two syntactic principles for sound signatures of primitive types introduced in Section VI-B are justified by the need to establish Lemma 5 Principle (P1) is necessary because we cannot assume anything about two invocations of an arbitrary partial function $\delta$, except that given syntactically equal arguments, if it produces results, then those results are syntactically equal. Principle (P2) is justified because any two invocations of $\delta$ are observationally equivalent at $\top$ (like any computation in general), so the actual relation between the arguments does not matter. For any primitive operator signature that does not abide to either $(\mathrm{P} 1)$ or $(\mathrm{P} 2)$, it is possible to devise a $\delta$ that violates Lemma 5

Consequently, $\mathrm{Ob}_{\mathrm{SEC}}^{\langle\rangle}$with primitive types is a sound security-typed language, i.e. all well-typed programs satisfy PRNI (Theorem 4).

\section{F. Illustration}

In Section III we gave informal examples of secure and insecure programs with respect to PRNI. Now, armed with Theorem 4 and the definitions for primitive types, we can formally check if a given program is secure by typechecking it. The prototype implementation of $\mathrm{Ob}_{\mathrm{SEC}}^{\langle\rangle}$features a number of examples and allows one to try out the language and typechecker. In this section, we unfold the reasoning underlying the proof of Theorem 4 for a specific example, in order to illustrate the technical details of PRNI and the relational interpretation of object types, including primitive signatures.

To alleviate notation, we omit unused type parameters in method declarations and type instantiations in method invocations. Note that we introduce the Unit type with its unique unit primitive value.

We illustrate polymorphic declassification by considering type and variable environments $\Delta \triangleq X$ : String..StringLen and $\Gamma \triangleq x$ : String $\triangleleft X$. We discuss two possible formal definitions for StringLen, either using standard method signatures, or using primitive signatures:

1) $\operatorname{Obj}(\alpha)$. [length : Unit $\rightarrow$ Int $\left._{\mathrm{L}}\right]$

2) $\operatorname{Obj}(\alpha)$. [length : Unit $\triangleleft * \rightarrow$ Int $\triangleleft *$ ]

With definition 1) above, the program $x$.length(unit) has type $\operatorname{Int}_{\mathrm{L}}$; i.e. $\Delta ; \Gamma \vdash x$.length(unit) : Int $\mathrm{L}_{\mathrm{L}}$. Then, by Theorem 4 , we know that $\operatorname{PRNI}\left(\Delta, \Gamma, x\right.$.length(unit), Int $_{\mathrm{L}}$ ) holds; the program is secure for any public observer.

Let us unfold $\operatorname{PRNI}\left(\Delta, \Gamma, x\right.$.length(unit), Int $\left._{\mathrm{L}}\right)$ to verify why it holds. For any type substitution $X \mapsto$ $T \in \mathcal{D} \llbracket \Delta \rrbracket$ and equivalent value substitutions $(k, x \mapsto$ $\left.v_{1}, x \mapsto v_{2}\right) \in \mathcal{G} \llbracket \bullet, x \quad$ : String $\triangleleft T \rrbracket$, we have that $\left(k, v_{1}\right.$. length(unit) $), v_{2}$.length(unit) $) \in \mathcal{C} \llbracket$ Int $\triangleleft$ Int $\rrbracket$.

To verify this:
1) By $\left(k, x \mapsto v_{1}, x \mapsto v_{2}\right) \in \mathcal{G} \llbracket \bullet, x$ : String $\triangleleft T \rrbracket$ we know that $\left(k, v_{1}, v_{2}\right) \in \mathcal{V} \llbracket$ String $\triangleleft T \rrbracket$. Because $T<$ : StringLen, we have $\mathcal{V} \llbracket$ String $\triangleleft T \rrbracket \subseteq$ $\mathcal{V} \llbracket$ String $\triangleleft$ StringLen》 by a subtyping lemma, and hence $\left(k, v_{1}, v_{2}\right) \in \mathcal{V} \llbracket$ String $\triangleleft$ StringLen .

2) Then, instantiate the definition of $\mathcal{V} \llbracket$ String $\triangleleft$ StringLen $\rrbracket$ with length, $k, T$, unit, unit. Note that:

- length $\in$ StringLen

- $\operatorname{msig}(\bullet$, StringLen, length $)=$ Unit $_{\mathrm{L}} \rightarrow \operatorname{Int}_{\mathrm{L}}$

- $T \in$ String..StringLen, which follows from $X \mapsto$ $T \in \mathcal{D} \llbracket \Delta \rrbracket$

- and ( $k$, unit, unit) $\in \mathcal{V} \llbracket$ Unit $\triangleleft$ Unit $\rrbracket$ (by definition of $\mathcal{V} \llbracket P \triangleleft P \rrbracket)$,

Then $\left(k, v_{1}\right.$.length(unit), $v_{2}$.length(unit) $) \in \mathcal{C} \llbracket$ Int $\triangleleft$ Int $\rrbracket$.

With definition 2) above of StringLen, we apply the same steps until the instantiation of $\mathcal{V} \llbracket$ String $\triangleleft$ StringLen』. At this point, since length has a primitive signature, we have to consider the extended case for primitive type signatures from Figure 12. Instantiate it with length, $k$, unit, unit, and observe that $\operatorname{msig}(\bullet$, StringLen, length $)=$ Unit $\triangleleft * \rightarrow$ Int $\triangleleft *$. Then, given that ( $k$, unit, unit) $\in \quad \mathcal{V} \llbracket$ Unit $\triangleleft$ Unit , we have that $\left(k, v_{1}\right.$.length(unit), $v_{2}$.length(unit) $\in$ $\mathcal{C} \llbracket$ Int $\triangleleft \operatorname{rdecl}($ Unit $\triangleleft$ Unit, Int $) \rrbracket=\mathcal{C} \llbracket$ Int $\triangleleft \operatorname{Int} \rrbracket$.

\section{RELATED WORK}

Declassification. Expressive declassification policies were introduced by $\mathrm{Li}$ and Zdancewic [3] with the labels-asfunctions approach. They define two kinds of declassification policies: local and global. Local policies are concerned with one secret, while global policies express coordinated declassification of several secrets. Label operations in this approach rely on a semantic interpretation of declassification functions based on a general notion of program equivalence. In addition to the induced complexity, this precludes recursive policies.

The labels-as-types approach [5] uses type interfaces instead of functions to express declassification policies. This simplifies the concepts involved (label ordering is simply subtyping), making an implementation more easily realizable. The approach naturally support local policies. More advanced typing disciplines such as refinement types [13] could in principle be used to express global policies.

Conceptually, Cruz et al. [5] relate secure information flow with type abstraction, a connection also explored under different angles by Bowman and Ahmed [14] and Washburn and Weirich [15]. Bowman and Ahmed [14] translate the noninterference result of the Dependency Core Calculus (DCC) [16] to parametricity, while Washburn and Weirich [15] use information control mechanisms to ensure a generalized from of parametricity in presence of runtime type inspection.

The Decentralized Label Model (DLM) [17] of Jif enforces robust declassification [18]: restricting who can declassify values, using the integrity policy to ensure that the declassification is not triggered by conditions affected by an active attacker. Here, we do not model any notion of authority, focusing on the what dimension of declassification [2]. 
As noted by Sabelfeld and Sands [2], many declassification approaches of the what dimension can be expressed using partial equivalence relations to model the public observer knowledge. Here, we use the logical interpretation of security types (Figure 7) to specify the partial equivalence relation that a public observer can use to distinguish values and computations.

Label polymorphism. Support for label polymorphism in security-typed programming languages can be classified in two categories: static and dynamic label polymorphism. Static label polymorphism can either be provided via explicit syntactic constructions to introduce generic labels [6], or implicitly with constraint-based label inference [6, 7, 19]. The dynamic form of label polymorphism relies on labels as first-class entities that can be passed around like standard values [6, 20, 21].

The Jif language [6] supports all three forms of label polymorphism. It provides a direct syntax to introduce labels at the method and class levels, which can be constrained. Also, Jif features label inference: local variables are inferred to have a fresh generic label that is resolved using constraints from the context. Inferred fields and method arguments have default labels. In addition, Jif supports first-class labels. Our work focuses on the foundations of explicit declassification polymorphism, and currently does not address label inference and first-class labels. Because labels are types, label inference would boil down to fairly standard type inference; first-class labels however would require a notion of first-class types, which should be considered with care.

Sun et al. [19] design a constraint-based label inference mechanism for an object-oriented language with classes and inheritance. Classes and methods are label polymorphic. The programmer can rely on the inference mechanism to achieve label polymorphism or to specify generic labels at the class level; method-level explicit polymorphism is not considered. Stefan et al. [20, 21] provide label-polymorphism via firstclass labels much like Jif.

Declassification and Polymorphism. When present, the declassification mechanisms of the label-polymorphic proposals discussed above [6, 7, 19, 21] are completely orthogonal to label polymorphism. The polymorphic labels-as-types approach developed in this work allows us to reason about declassification and label polymorphism with the single and unified concept of standard types.

Our approach is closely related to that of Hicks et al. [4], which propose trusted declassification in an object-oriented language based on the DLM [17], where each label is composed of principals. Declassification is globally defined, associating principals to the trusted methods that can be used to declassify an expression to another principal. Because classes are polymorphic with respect to principals, this induces a form of implicit label polymorphism. More precisely, a class definition is checked at instantiation-time with the actual principals provided for the instantiation. This use-site polymorphism for principals is similar to our treatment of polymorphic primitive signatures (Section VI).

Tse and Zdancewic [22] propose certificate-based declas- sification and conditioned noninterference. They extend System $\mathrm{F}_{<\text {: }}$ with monadic labels similarly to DCC [16], using DLM [17]. Declassification is modeled as a read privilege that a principal is allowed to give to another principal in a certain context. Their work merges standard types with labels, principals and privileges in the same syntactic category of types. Since System $F_{<:}$supports type polymorphism, the language supports label polymorphism. However, it is not clear how to use label polymorphism to express polymorphic declassification in that setting.

Finally, the syntactic principles we introduce for primitive signatures are related to the work of $\mathrm{Li}$ and Zdancewic [3] on labels-as-functions. For local policies, the typing rules for integer primitive operators follow the same principles, but are more expressive. In particular, they provide typing rules for binary integer operators where one operand has an arbitrary declassification policy and the other operand is public; the resulting label is a functional composition of the operand label with a function wrapping the operator. As explained before, this semantic implication cannot be expressed with the labelsas-types approach, unless one is willing to consider much more advanced typing disciplines.

\section{CONCLUSION}

We extend relaxed noninterference in a labels-as-types approach to selective and expressive declassification in order to account for polymorphism. The proposed declassification polymorphism is novel and useful to precisely control declassification of polymorphic structures and to define procedures that are polymorphic over the declassification policies of their arguments. Bounded polymorphism further controls the guarantees and expectations of clients and providers with respect to declassification. Bringing type-based declassification to realworld programming also requires addressing the issue of primitive types, which were ignored in prior work. For this we introduce a novel form of ad-hoc polymorphism. We formalize the approach, prove its soundness, and provide a prototype implementation.

This work provides a necessary and solid basis to integrate type-based declassification in existing languages. A particularly appealing alternative is to study the realization of our approach in Scala: its type system is expressive enough to encode bounded polymorphic declassification, and adjusting the typechecker to account for security levels (i.e. the additional rule for method invocation) should be achievable via a compiler plugin.

\section{ACKNOWLEDGMENT}

We thank Cătălin Hriţcu and the anonymous reviewers for their detailed comments and suggestions. 


\section{REFERENCES}

[1] D. Volpano, C. Irvine, and G. Smith, "A sound type system for secure flow analysis," Journal of Computer Security, vol. 4, no. 2-3, pp. 167-187, Jan. 1996.

[2] A. Sabelfeld and D. Sands, "Declassification: Dimensions and principles," Journal of Computer Security, vol. 17, no. 5, pp. 517-548, 2009.

[3] P. Li and S. Zdancewic, "Downgrading policies and relaxed noninterference," in Proceedings of the 32nd ACM SIGPLAN-SIGACT Symposium on Principles of Programming Languages (POPL 2005). Long Beach, CA, USA: ACM Press, Jan. 2005, pp. 158-170.

[4] B. Hicks, D. King, P. McDaniel, and M. Hicks, "Trusted declassification: high-level policy for a security-typed language," in Proceedings of the workshop on Programming Languages and Analysis for Security (PLAS 2006, 2006, pp. 65-74.

[5] R. Cruz, T. Rezk, B. Serpette, and É. Tanter, "Type abstraction for relaxed noninterference," in Proceedings of the 31st European Conference on Object-Oriented Programming (ECOOP 2017), ser. Leibniz International Proceedings in Informatics (LIPIcs), P. Müller, Ed., vol. 74. Barcelona, Spain: Schloss Dagstuhl-LeibnizZentrum fuer Informatik, Jun. 2017, pp. 7:1-7:27.

[6] A. C. Myers, "Jif homepage," http://www.cs.cornell.edu/jif/, accessed March 2019.

[7] F. Pottier and V. Simonet, "Information flow inference for ML," ACM Transactions on Programming Languages and Systems, vol. 25, no. 1, pp. 117-158, 2003.

[8] M. Abadi and L. Cardelli, A Theory of Objects. Springer-Verlag, 1996.

[9] A. Igarashi, B. C. Pierce, and P. Wadler, "Featherweight Java: a minimal core calculus for Java and GJ," $A C M$ Transactions on Programming Languages and Systems, vol. 23, no. 3, pp. 396-450, 2001.

[10] T. Rompf and N. Amin, "Type soundness for dependent object types (DOT)," in Proceedings of the 31st ACM SIGPLAN Conference on Object-Oriented Programming, Systems, Languages, and Applications, (OOPSLA 2016), part of SPLASH 2016, E. Visser and Y. Smaragdakis, Eds. Amsterdam, The Netherlands: ACM Press, Oct. 2016, pp. 624-641.

[11] A. Ahmed, "Step-indexed syntactic logical relations for recursive and quantified types," in Proceedings of the 15th European Symposium on Programming Languages and Systems (ESOP 2006), ser. Lecture Notes in Computer Science, P. Sestoft, Ed., vol. 3924. Vienna, Austria: Springer-Verlag, Mar. 2006, pp. 69-83.

[12] S. Zdancewic, "Programming languages for information security," Ph.D. dissertation, Cornell University, Aug. 2002.

[13] P. M. Rondon, M. Kawaguchi, and R. Jhala, "Liquid types," in Proceedings of the ACM SIGPLAN Conference on Programming Language Design and Implementation (PLDI 2008), R. Gupta and S. P. Amarasinghe, Eds.
ACM Press, Jun. 2008, pp. 159-169.

[14] W. J. Bowman and A. Ahmed, "Noninterference for free," in Proceedings of the 20th ACM SIGPLAN Conference on Functional Programming (ICFP 2015). Vancouver, Canada: ACM Press, Aug. 2015, pp. 101-113.

[15] G. Washburn and S. Weirich, "Generalizing parametricity using information-flow," in Proceedings of the 20th IEEE Symposium on Logic in Computer Science (LICS 2005). Chicago, IL, USA: IEEE Computer Society Press, Jun. 2005, pp. 62-71.

[16] M. Abadi, A. Banerjee, N. Heintze, and J. G. Riecke, "A core calculus of dependency," in Proceedings of the 26th ACM Symposium on Principles of Programming Languages (POPL 99). San Antonio, TX, USA: ACM Press, Jan. 1999, pp. 147-160.

[17] A. C. Myers and B. Liskov, "Protecting privacy using the decentralized label model," ACM Transactions on Software Engineering and Methodology, vol. 9, pp. 410442, Oct. 2000.

[18] S. Zdancewic and A. C. Myers, "Robust declassification," in Proceedings of the 14th IEEE Computer Security Foundations Workshop (CSFW 2001). Cape Breton, Nova Scotia, Canada: IEEE Computer Society Press, Jun. 2001, pp. 15-23.

[19] Q. Sun, A. Banerjee, and D. A. Naumann, "Modular and constraint-based information flow inference for an objectoriented language," in Proceedings of the 11th Static Analysis Symposium (SAS 2004), ser. Lecture Notes in Computer Science, R. Giacobazzi, Ed., vol. 3148. Verona, Italy: Springer-Verlag, Aug. 2004, pp. 84-99.

[20] D. Stefan, A. Russo, J. C. Mitchell, and D. Mazières, "Flexible dynamic information flow control in Haskell," in Proceedings of the 4th ACM Symposium on Haskell. ACM Press, 2011, pp. 95-106.

[21] D. Stefan, D. Mazières, J. C. Mitchell, and A. Russo, "Flexible dynamic information flow control in the presence of exceptions," Journal of Functional Programming, vol. 27, 2017.

[22] S. Tse and S. Zdancewic, "A design for a securitytyped language with certificate-based declassification," in Proceedings of the 14th European Symposium on Programming Languages and Systems (ESOP 2005), ser. Lecture Notes in Computer Science, S. Sagiv, Ed., vol. 2986. Berlin, Heidelberg: Springer-Verlag, 2005, pp. 279-294. 
APPENDIX A

FULL SYNTAX AND SEMANTICS

A. Syntax

$\begin{array}{rlrl}e & ::=v|e \cdot m(e)| e . m\langle U\rangle(e)|x| & \mathrm{p} \text { (terms) } \\ o & ::=[z: S \Rightarrow \overline{m(x) e}] & & \text { (objects) } \\ v & :=o \mid \mathrm{p} & & \text { (values) } \\ T & ::=O|\alpha| P & & \text { (types) } \\ U, A, B & ::=T \mid X & & \text { (policies) } \\ O & ::=\mathbf{O b j}(\alpha) \cdot[\overline{m: M}] & & \text { (object type) } \\ R & ::=[\overline{m: M}] & & \text { (record types) } \\ M & ::=\langle X: A . . B\rangle S \rightarrow S \mid I & & \text { (method signature) } \\ I & ::=P \triangleleft * \rightarrow P \triangleleft * & & \text { (primitive signatures) } \\ S & ::=T \triangleleft U \mid P \triangleleft * & & \text { (security types) } \\ P & ::=\text { (eg. Int) } & & \text { (primitive type) }\end{array}$

Fig. 13. Ob $\mathrm{SEC}_{\mathrm{SEC}}^{\langle\text {Full Syntax }}$ 


$$
\begin{array}{rlrl}
\Gamma:: & =\bullet \mid \Gamma, x: S & & \text { (type environment) } \\
\Delta & ::=\bullet \mid \Delta, X: A . . B & & \text { (generic type variable environment) } \\
\Phi::=\bullet|\Phi, \alpha<: \beta| \Phi, P<: \beta & \text { (subtyping environment) } \\
\Delta_{\mathrm{ok}}:: & =\bullet\left|\Delta_{\mathrm{ok}}, \alpha\right| \Delta_{\mathrm{ok}}, X: A . . B & & \text { (type variable environment) } \\
\Sigma:: & =\bullet \mid \Sigma, \alpha \triangleq O & & \text { (type definition environment) }
\end{array}
$$

The type variable environment $\Delta_{\mathrm{ok}}$ keeps track of the type variable definitions, both self type variables and generic type variables. This environment is not directly used in the static semantics presented in the body of the paper. Recall that in the typing judgment $\Delta ; \Gamma \vdash e: S$ of Figure 5 , we extend the typing environment $\Gamma$ with closed types regarding self type variables. For this reason we use $\Delta$ instead of $\Delta_{\text {ok }}$. Note that $\Delta$ just keeps track of generic type variable definition. Then, the environment $\Delta_{\mathrm{ok}}$ is just an auxiliary mechanism to verify that an object type is well-formed (Figure 17) 


\section{Type Equivalence}

The type equivalence judgment (Figure 14] is essentially the same that the work of Cruz et al. [5], with minor modifications to the rule (O-congr) to support type variables.

Two types are equivalent (Figure [14p if the equivalence can be derived through the congruence induced by rules (Alpha-Eq) and (Fold-Unfold). For example:

$\operatorname{Obj}(\alpha) \cdot[m:\langle X: \alpha . . \top\rangle \alpha \rightarrow \alpha] \equiv \operatorname{Obj}(\beta) \cdot[m:\langle X: \beta . . \top\rangle \beta \rightarrow \beta]$

$\operatorname{Obj}(\alpha) .[m:\langle X: \alpha . . \top\rangle \top \rightarrow \alpha] \equiv \operatorname{Obj}(\alpha) .[m:\langle X: \alpha . . \top\rangle \top \rightarrow \operatorname{Obj}(\beta) .[m:\langle Y: \beta . . \top\rangle \top \rightarrow \beta]]$

$U \equiv U$

$$
\begin{gathered}
(\text { Sym }) \frac{U_{1} \equiv U_{2}}{U \equiv U} \quad \text { (Refl) } \frac{U_{1} \equiv U_{2}}{U_{2} \equiv U_{1}} \quad \text { (Trans) } \frac{U_{2} \equiv U_{3}}{U_{1} \equiv U_{3}} \\
\text { (O-Congr) } \left.\frac{L_{i} \equiv L_{i}^{\prime} \quad U_{i} \equiv U_{i}^{\prime} \quad S_{1 i} \equiv S_{1 i}^{\prime} \quad S_{2 i} \equiv S_{2 i}^{\prime}}{\mathbf{O b j}(\alpha) \cdot\left[m:\langle X: A . . B\rangle S_{1} \rightarrow S_{2}\right] \equiv \mathbf{O b j}(\alpha) \cdot\left[m:\left\langle X: L^{\prime} . U^{\prime}\right\rangle S_{1}^{\prime} \rightarrow S_{2}^{\prime}\right.}\right] \\
\text { (Alpha-Eq) } \frac{O \triangleq \mathbf{O b j}(\alpha) \cdot[\overline{m: M}] \quad \beta \text { fresh }}{O \equiv O[\beta / \alpha]} \quad \text { (Fold-Unfold) } \frac{}{O \equiv O[O / \alpha]}
\end{gathered}
$$

$S \equiv S$

$$
\frac{T_{1} \equiv T_{2} \quad U_{1} \equiv U_{2}}{T_{1} \triangleleft U_{1} \equiv T_{2} \triangleleft U_{2}}
$$

Fig. 14. $\mathrm{Ob}_{\mathrm{SEC}}^{\langle\rangle}$: Type equivalence 


\section{Subtyping}

$\Delta ; \Phi \vdash U<: U$

$$
\begin{aligned}
& \operatorname{meths}(P)=R_{1} \quad O \triangleq \mathbf{O b j}(\beta) \cdot R_{2} \\
& (\mathrm{SPObj}) \frac{\Delta ; \Phi, P<: \beta \vdash R_{1}<: R_{2}}{\Delta ; \Phi \vdash P<: O}(\text { SPVar }) \frac{P<: \beta \in \Phi}{\Delta ; \Phi \vdash P<: \beta} \\
& O_{1} \triangleq \mathbf{O b j}(\alpha) \cdot R_{1} \quad O_{2} \triangleq \mathbf{O b j}(\beta) \cdot R_{2} \\
& (\mathrm{SObj}) \frac{\Delta ; \Phi, \alpha<: \beta \vdash R_{1}<: R_{2}}{\Delta ; \Phi \vdash O_{1}<: O_{2}} \quad(\mathrm{SGVar} 1) \frac{X: A . . B \in \Delta}{\Delta ; \Phi \vdash X<: B} \quad(\mathrm{SGVar} 2) \frac{X: A . . B \in \Delta}{\Delta ; \Phi \vdash A<: X} \\
& \text { (SVar) } \frac{\alpha<: \beta \in \Phi}{\Delta ; \Phi \vdash \alpha<: \beta} \quad(\text { SSubEq }) \frac{O_{1} \equiv O_{2}}{\Delta ; \Phi \vdash O_{1}<: O_{2}} \\
& \text { (STrans) } \frac{\Delta ; \Phi \vdash U_{1}<: U_{2} \quad \Delta ; \Phi \vdash U_{2}<: U_{3}}{\Delta ; \Phi \vdash U_{1}<: U_{3}}
\end{aligned}
$$

$\Delta ; \Phi \vdash R_{1}<: R_{2}$

$$
(\mathrm{SR}) \frac{\overline{m^{\prime}} \subseteq \bar{m} \quad m_{i}=m_{j}^{\prime} \Longrightarrow \Delta ; \Phi \vdash M<: M^{\prime}}{\Delta ; \Phi \vdash[\overline{m: M}]<:\left[\overline{m^{\prime}: M^{\prime}}\right]}
$$

$\Delta ; \Phi \vdash M<: M$

$$
\begin{array}{cc}
\Delta ; \Phi \vdash B^{\prime}<: B & \Delta ; \Phi \vdash A<: A^{\prime} \\
\text { (SM) } \frac{\Delta, X: A^{\prime} . . B^{\prime} ; \Phi \vdash S_{1}^{\prime}<: S_{1}}{\Delta, X: A^{\prime} . . B^{\prime} ; \Phi \vdash S_{2}<: S_{2}^{\prime}} & \text { (SImpl) } \frac{}{\Delta ; \Phi \vdash I<: I}
\end{array}
$$

$\Delta ; \Phi \vdash S<: S$

$$
\text { (TSubST) } \frac{\Delta ; \Phi \vdash T_{1}<: T_{2} \quad \Delta ; \Phi \vdash U_{1}<: U_{2}}{\Delta ; \Phi \vdash T_{1} \triangleleft U_{1}<: T_{2} \triangleleft U_{2}}
$$

Fig. 15. $\mathrm{Ob}_{\mathrm{SEC}}^{\langle\rangle}\langle$Subtyping rules 


\section{E. Standard well formedness of types and environments}

The Fig. 16 and Fig. 17 show the rules for well formedness of environments and types respectively.

Since $\Delta$ match the syntax of $\Delta_{\text {ok }}$, we can use the judgments $\Delta_{\text {ok }} \vdash U$ and $\Delta_{\text {ok }} \vdash S$ with $\Delta$ and they hold if $U$ (an $S$ respectively) are closed with respecto to self type variables $\alpha$.

\section{$\Delta \vdash \Gamma$}

$$
\frac{\Delta \vdash \Gamma \quad \Delta \models S \quad x \notin \operatorname{dom}(\Gamma)}{\Delta \vdash \cdot \quad \frac{\Delta \vdash \Gamma, x}{\Delta}}
$$

$\vdash \Delta$

$$
\frac{\vdash}{\vdash \cdot} \frac{\vdash \quad X \notin \operatorname{dom}(\Delta) \quad \Delta \vdash A \quad \Delta \vdash B}{\vdash \Delta, X: A . . B}
$$

$\vdash \Phi$

$\frac{\vdash \Phi \quad \alpha_{i} \notin \operatorname{dom}(\Phi) \cup \operatorname{cod}(\Phi)}{\vdash \cdot} \frac{\vdash, \alpha_{1}<: \alpha_{2}}{\vdash \cdot \quad \alpha_{2} \notin \operatorname{dom}(\Phi) \cup \operatorname{cod}(\Phi) \quad \models P} \frac{\vdash \Phi, P<: \alpha_{2}}{\vdash}$

Fig. 16. $\mathrm{Ob}_{\mathrm{SEC}}^{\langle\rangle}$: Environments well-formedness

$\Delta_{\mathrm{ok}} \vdash U$

$$
\begin{aligned}
& O \equiv \mathbf{O b j}(\alpha) \cdot\left[m:\langle X: A . . B\rangle S_{1} \rightarrow S_{2}\right] \\
& \left(i \neq j \Longrightarrow m_{i} \neq m_{j}\right) \\
& \Delta_{\mathrm{ok}}, \alpha \vdash A \quad \Delta_{\mathrm{ok}}, \alpha \vdash B \\
& \frac{\models P}{\Delta_{\mathrm{ok}} \vdash P} \quad \frac{X \in \Delta_{\mathrm{ok}}}{\Delta_{\mathrm{ok}} \vdash X} \quad \frac{\alpha \in \Delta_{\mathrm{ok}}}{\Delta_{\mathrm{ok}} \vdash \alpha} \quad \frac{\Delta_{\mathrm{ok}}, \alpha, X: A . . B \vdash S_{1 i} \quad \Delta_{\mathrm{ok}}, \alpha, X: A . . B \vdash S_{2 i}}{\Delta_{\mathrm{ok}} \vdash O}
\end{aligned}
$$

$\Delta_{\mathrm{ok}} \vdash S$

$$
\frac{\Delta_{\mathrm{ok}} \vdash T \quad \Delta_{\mathrm{ok}} \vdash U}{\Delta_{\mathrm{ok}} \vdash T \triangleleft U}
$$

Fig. 17. $\mathrm{Ob}_{\mathrm{SEC}}^{\langle\rangle}$: Well-formedness of types 


$$
\text { soundsig }\left(\left\langle_{-}\right\rangle P_{1} \triangleleft U_{1} \rightarrow T_{2} \triangleleft U_{2}\right) \Longleftrightarrow P_{1}=U_{1} \vee U_{2}=\top
$$

Fig. 18 shows extended subtyping judgments that are the same the ones of Figure 15, except that the judgment $\Delta ; \Phi \vdash M$ $M$ adds the rule (IG) justifying that is OK to declassify a primitive signature with a concrete sound signature.

The judgment $\Delta=S$ holds if the type $S$ is a closed type and a well-formed security type.

Having formalized well-formedness of environments and types, we assume them in most definitions.

$$
\Delta ; \Phi \vdash U \triangleleft U
$$

$\Delta ; \Phi \vdash R_{1} \triangleleft R_{2}$

$$
\Delta ; \Phi \vdash M \triangleleft M
$$

$$
\cdots \quad(\mathrm{IG}) \frac{\Delta ; \Phi \vdash T_{1} \triangleleft P_{1} \quad \Delta ; \Phi \vdash P_{2} \triangleleft T_{2} \quad \text { soundsig }\left(\left\langle_{-}\right\rangle T_{1} \triangleleft U_{1} \rightarrow T_{2} \triangleleft U_{2}\right)}{\Delta ; \Phi \vdash P_{1} \triangleleft * \rightarrow P_{2} \triangleleft * \triangleleft\left\langle \_\right\rangle T_{1} \triangleleft U_{1} \rightarrow T_{2} \triangleleft U_{2}}
$$

$\Delta ; \Phi \vdash S \triangleleft S$

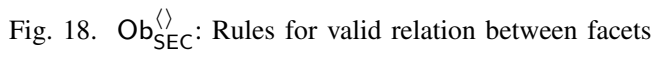

$\Delta ; \Sigma \vdash_{\triangleleft} U$

$$
\frac{\begin{array}{l}
O \equiv \boldsymbol{O b j}(\alpha) \cdot[\overline{m: M}] \\
\Delta ; \Sigma, \alpha: O \vdash_{\triangleleft} M
\end{array}}{\Delta ; \Sigma \vdash_{\triangleleft} O} \frac{}{\Delta ; \Sigma \vdash_{\triangleleft} \alpha} \frac{}{\Delta ; \Sigma \vdash_{\triangleleft} X} \frac{}{\Delta ; \Sigma \vdash_{\triangleleft} P}
$$

$\Delta ; \Sigma \vdash_{\triangleleft} M$

$$
\frac{\Delta, X: A . . B ; \Sigma \vdash_{\triangleleft} S_{1} \quad \Delta, X: A . . B ; \Sigma \vdash_{\triangleleft} S_{2}}{\Delta ; \Sigma \vdash_{\triangleleft}\langle X: A . . B\rangle S_{1} \rightarrow S_{2}} \quad \frac{}{\Delta ; \Sigma \vdash_{\triangleleft} I}
$$

$\Delta ; \Sigma \vdash_{\triangleleft} S$

$$
\frac{\Delta ; \Sigma \vdash_{\triangleleft} T \quad \Delta ; \Sigma \vdash_{\triangleleft} U \quad \Delta ; \cdot \vdash \Sigma[T] \triangleleft \Sigma[U]}{\Delta ; \Sigma \vdash_{\triangleleft} T \triangleleft U}
$$

$\Delta \models S$

$$
\frac{\Delta \vdash S \quad \Delta ; \cdot \vdash_{\triangleleft} S}{\Delta=S}
$$

Fig. 19. $\mathrm{Ob}_{\mathrm{SEC}}^{\langle\rangle}$: Well-formedness of security types facet-wise 
$\mathrm{ub}(\Delta, U)=T$

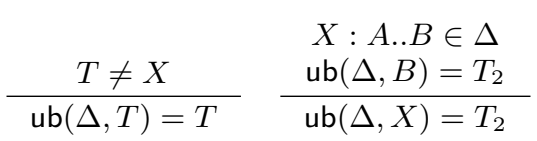

$\Delta \vdash m \in U$

$\frac{O \triangleq \mathbf{O b j}(\alpha) \cdot[\overline{m: M}]}{\Delta \vdash m_{i} \in O} \quad \frac{\operatorname{meths}(P)=[\overline{m: I}]}{\Delta \vdash m_{i} \in P} \quad \frac{\Delta \vdash m \in \mathrm{ub}(\Delta, X)}{\Delta \vdash m \in X}$

$\operatorname{msig}(\Delta, U, m)=M$

$$
\begin{gathered}
\frac{O \triangleq \mathbf{O b j}(\alpha) \cdot\left[\overline{m: M_{i}}\right]}{\operatorname{msig}\left(, O, m_{i}\right)=M_{i}[O / \alpha]} \quad \frac{\operatorname{meths}(P)=[\overline{m: I}]}{\operatorname{msig}\left(,, P, m_{i}\right)=I_{i}} \\
\frac{\operatorname{msig}(\Delta, X, m)=\operatorname{msig}\left(\_, \mathrm{ub}(\Delta, X), m\right)}{}
\end{gathered}
$$

methimpl $(o, m)=x . e$

$$
\frac{o \triangleq[z: S \Rightarrow \overline{m(x) e}]}{\operatorname{methimpl}\left(o, m_{i}\right)=x \cdot e_{i}}
$$

$\Delta \vdash U \in A . . B$

$$
\frac{\Delta ; \bullet \vdash<: U \quad \Delta ; \bullet \vdash U<: B}{\Delta \vdash U \in A . . B}
$$

$\operatorname{rdecl}(P \triangleleft U, P)=U$

$$
\operatorname{rdecl}\left(P_{1} \triangleleft U_{1}, P_{2}\right)= \begin{cases}P_{2} & P_{1}=U_{1} \\ \top & \text { otherwise }\end{cases}
$$

Fig. 20. $\mathrm{Ob}_{\mathrm{SEC}}^{\langle\rangle}$: Auxiliary definitions 


\section{H. Typing}

$\Delta ; \Gamma \vdash e: S$

$$
\begin{aligned}
& \text { (TVar) } \frac{x \in \operatorname{dom}(\Gamma)}{\Delta ; \Gamma \vdash x: \Gamma(x)} \quad \text { (TSub) } \frac{\Delta ; \Gamma \vdash e: S^{\prime} \quad \vdash S^{\prime}<: S}{\Delta ; \Gamma \vdash e: S} \\
& S \triangleq T \triangleleft U \quad \operatorname{msig}\left(\_, T, m_{i}\right)=\left\langle X: A_{i} . . B_{i}\right\rangle S_{i}^{\prime} \rightarrow S_{i}^{\prime \prime} \\
& \text { (TPrim) } \frac{P=\Delta_{\mathrm{p}}(\mathrm{p})}{\Gamma \vdash \mathrm{p}: P \triangleleft P} \quad(\mathrm{TObj}) \frac{\Delta, X: A_{i} . . B_{i} ; \Gamma, z: S, x: S_{i}^{\prime} \vdash e_{i}: S_{i}^{\prime \prime}}{\Delta ; \Gamma \vdash[z: S \Rightarrow \overline{m(x) e}]: S} \\
& \Delta ; \Gamma \vdash e_{1}: T \triangleleft U \quad \Delta \vdash m \in U \quad \operatorname{msig}(\Delta ; U, m)=\langle X: A . . B\rangle S_{1} \rightarrow S_{2} \\
& (\mathrm{TmD}) \frac{\Delta \vdash U^{\prime} \in A . . B \quad \frac{\Delta ; \Gamma \vdash e_{2}: S_{1}\left[U^{\prime} / X\right]}{\Delta ; \Gamma \vdash e_{1} . m\left\langle U^{\prime}\right\rangle\left(e_{2}\right): S_{2}\left[U^{\prime} / X\right]}}{\Delta} \\
& \Delta ; \Gamma \vdash e_{1}: T \triangleleft U \quad \Delta \vdash m \notin U \quad \operatorname{msig}\left({ }_{-}, T, m\right)\langle X: A . . B\rangle S_{1} \rightarrow T_{2} \triangleleft U_{2} \\
& (\mathrm{TmH}) \frac{\Delta \vdash U^{\prime} \in A . . B}{\Delta ; \Gamma \vdash e_{1} . m\left\langle U^{\prime}\right\rangle\left(e_{2}\right): T_{2}\left[U^{\prime} / X\right] \triangleleft \top} \\
& \Delta ; \Gamma \vdash e_{1}: T \triangleleft U \quad \Delta \vdash m \in U \quad \operatorname{msig}(\Delta, U, m)=P_{1} \triangleleft * \rightarrow P_{2} \triangleleft * \\
& (\mathrm{TPmD}) \frac{\Delta ; \Gamma \vdash e_{2}: P_{1} \triangleleft U_{1} \quad \operatorname{rdecl}\left(P_{1} \triangleleft U_{1}, P_{2}\right)=P_{2}^{\prime}}{\Delta ; \Gamma \vdash e_{1} \cdot m\left(e_{2}\right): P_{2} \triangleleft P_{2}^{\prime}} \\
& \Delta ; \Gamma \vdash e_{1}: T \triangleleft U \quad \Delta \vdash m \notin U \quad \operatorname{msig}(\Delta, T, m)=P_{1} \triangleleft * \rightarrow P_{2} \triangleleft * \\
& \text { (TPmH) } \frac{\Delta ; \Gamma \vdash e_{2}: P_{1} \triangleleft U_{1}}{\left.\Delta ; \Gamma \vdash e_{1}, e_{2}\right): P_{2} \triangleleft \top} \\
& \Delta ; \Gamma \vdash e_{1} \cdot m\left(e_{2}\right): P_{2} \triangleleft \top
\end{aligned}
$$

Fig. 21. $O \mathrm{~b}_{\mathrm{SEC}}^{\langle\rangle}$: Static semantics 


\section{Dynamic semantics}

$E::=[]|\operatorname{E} \cdot m(e)| v \cdot m(E)$ (evaluation contexts)

(EMInvO) $\frac{o \triangleq\left[z:_{-} \Rightarrow_{-}\right] \quad \text { methimpl }(o, m)=x . e}{E\left[o . m\left\langle_{-}\right\rangle(v)\right] \longmapsto E[e[o / z][v / x]]}$

$\left(\right.$ EMInvP) $\frac{E\left[\mathrm{p}_{1} . m\left(\mathrm{p}_{2}\right)\right] \longmapsto E\left[\theta\left(m, \mathrm{p}_{1}, \mathrm{p}_{2}\right)\right]}{\longmapsto}$

Fig. 22. $\mathrm{Ob}_{\mathrm{SEC}}^{\langle\rangle}$: Full Dynamic semantics 


\section{J. Simple Type System}

Figure 23 and Figure 24 define a simple type system and simple subtyping judgments respectively that do not take into account the declassification type. The typing judgment $\Gamma \vdash_{\mathrm{sf}} e: S$ uses the subtyping judgment $\Phi \vdash_{\mathrm{sf}} S<: S$. The definition of the judgment $\Phi \vdash_{\mathrm{sf}} T<: T$ is straightforward and then omit here.

$\Gamma \vdash_{\mathrm{sf}} e: S$

$$
\begin{aligned}
& (\mathrm{T} 1 \mathrm{Var}) \frac{x \in \operatorname{dom}(\Gamma)}{\Gamma \vdash_{\mathrm{sf}} x: \Gamma(x)} \quad(\mathrm{T} 1 \mathrm{Sub}) \frac{\Gamma \vdash_{\mathrm{sf}} e: S^{\prime} \vdash_{\mathrm{sf}} S^{\prime}<: S}{\Gamma \vdash_{\mathrm{sf}} e: S} \quad(\mathrm{~T} 1 \text { Prim }) \frac{P=\Theta(\mathrm{p})}{\Gamma \vdash_{\mathrm{sf}} \mathrm{p}: P \triangleleft P} \\
& (\mathrm{~T} 1 \mathrm{Obj}) \frac{\operatorname{msig}\left(\_, T, m_{i}\right)=\left\langle_{\_}\right\rangle S_{i}^{\prime} \rightarrow S_{i}^{\prime \prime} \quad \Gamma, z: S, x_{i}: S_{i}^{\prime} \vdash_{\mathrm{sf}} e_{i}: S_{i}^{\prime \prime}}{\Gamma \vdash_{\mathrm{sf}}[z: S \Rightarrow \overline{m(x) e}]: S} \\
& (\mathrm{~T} 1 \mathrm{mI}) \frac{\Gamma \vdash_{\mathrm{sf}} e_{1}: T \triangleleft U \quad \operatorname{msig}\left({ }_{-}, T, m\right)=\left\langle_{-}\right\rangle S_{1} \rightarrow S_{2} \quad \Gamma \vdash_{\mathrm{sf}} e_{2}: S_{1}}{\Gamma \vdash_{\mathrm{sf}} e_{1} \cdot m\left\langle_{-}\right\rangle\left(e_{2}\right): S_{2}} \\
& (\mathrm{~T} 1 \mathrm{PmI}) \frac{\Gamma \vdash_{\mathrm{sf}} e_{1}: T \triangleleft U \quad \operatorname{msig}\left({ }_{-}, T, m\right)=P_{1} \triangleleft * \rightarrow P_{2} \triangleleft * \quad \Gamma \vdash_{\mathrm{sf}} e_{2}: P_{1} \triangleleft *}{\Gamma \vdash_{\mathrm{sf}} e_{1} \cdot m\left(e_{2}\right): P_{2} \triangleleft *}
\end{aligned}
$$

$\Gamma \vdash_{1} e: T$

$$
\frac{\Gamma \vdash_{\mathrm{sf}} e: T \triangleleft U}{\Gamma \vdash_{1} e: T}
$$

Fig. 23. $\mathrm{Ob}_{\mathrm{SEC}}^{\langle\rangle}$simple typing, defined in terms of single-facet typing

$\Phi \vdash_{\mathrm{sf}} T<: T$

$\Phi \vdash_{\mathrm{sf}} S<: S$

$\frac{\Phi \vdash_{\mathrm{sf}} T_{1}<: U_{1}}{\Phi \vdash_{\mathrm{sf}} T_{1} \triangleleft U_{1}<: T_{2} \triangleleft U_{2}}$

Fig. 24. $\mathrm{Ob}_{\mathrm{SEC}}^{\langle\rangle}$simple subtyping 


\section{APPENDIX B}

UNARY MODEL

We present the logical predicate for safety (Figure 25. This logical predicate gives a safety meaning to a safety type, hence the declassification type does not play any role in the definitions.

$$
\begin{gathered}
\mathcal{V}_{k} \llbracket P \rrbracket=\{\mathrm{p} \mid \\
\left(\forall j<k . v \in \mathcal{V}_{j} \llbracket P \rrbracket \wedge\right. \\
\left(\forall m \in P, \mathrm{p}^{\prime} . \operatorname{msig}(\bullet, P, m)=P^{\prime} \triangleleft * \rightarrow P^{\prime \prime} \triangleleft *\right. \\
\mathrm{p}^{\prime} \in \mathcal{V}_{j} \llbracket P^{\prime} \rrbracket \Longrightarrow \\
\Longrightarrow
\end{gathered}
$$

Fig. 25. Unary logical relation for safety 


\section{APPENDIX C}

TYPE SAFETY

Definition 1 (Safety). $\operatorname{safe}(e) \Longleftrightarrow \forall e^{\prime} . e \longmapsto{ }^{*} e^{\prime} \Longrightarrow e^{\prime}=v$ or $\exists e^{\prime \prime} . e^{\prime} \longmapsto e^{\prime \prime}$

Definition 4 (Semantic typing). $\models e: T \triangleleft U \Longleftrightarrow \forall k \geq 0 . e \in \mathcal{C}_{k} \llbracket T \rrbracket$.

Lemma 6 (Semantic type safety). $=e: S \Longrightarrow \operatorname{safe}(e)$

Proof. The proof follows directly from definitions 1 and 4 .

Lemma 7 (Type Safety). $\Gamma \vdash e: S \Longrightarrow \Gamma \models e: S$

Proof. The proof is by induction on the typing derivation of $e$. The different case with respect to the proof of type safety of $\mathrm{Ob}_{\mathrm{SEC}}[5]$ is the case (TPrim). For that case we only need to assume that $\Theta$ and $\theta$-which are parameters of the language-agree on the specification and implementation of all primitive types and their operations.

Theorem 1 (Syntactic type safety). $\vdash e: S \Longrightarrow \operatorname{safe}(e)$

Proof. This follows directly from Lemmas 6 and 7 


\section{APPENDIX D}

\section{POLYMORPHIC RELAXED NONINTERFERENCE}

\section{A. Logical relation for PRNI}

Figure 26 shows the full logical relation for PRNI

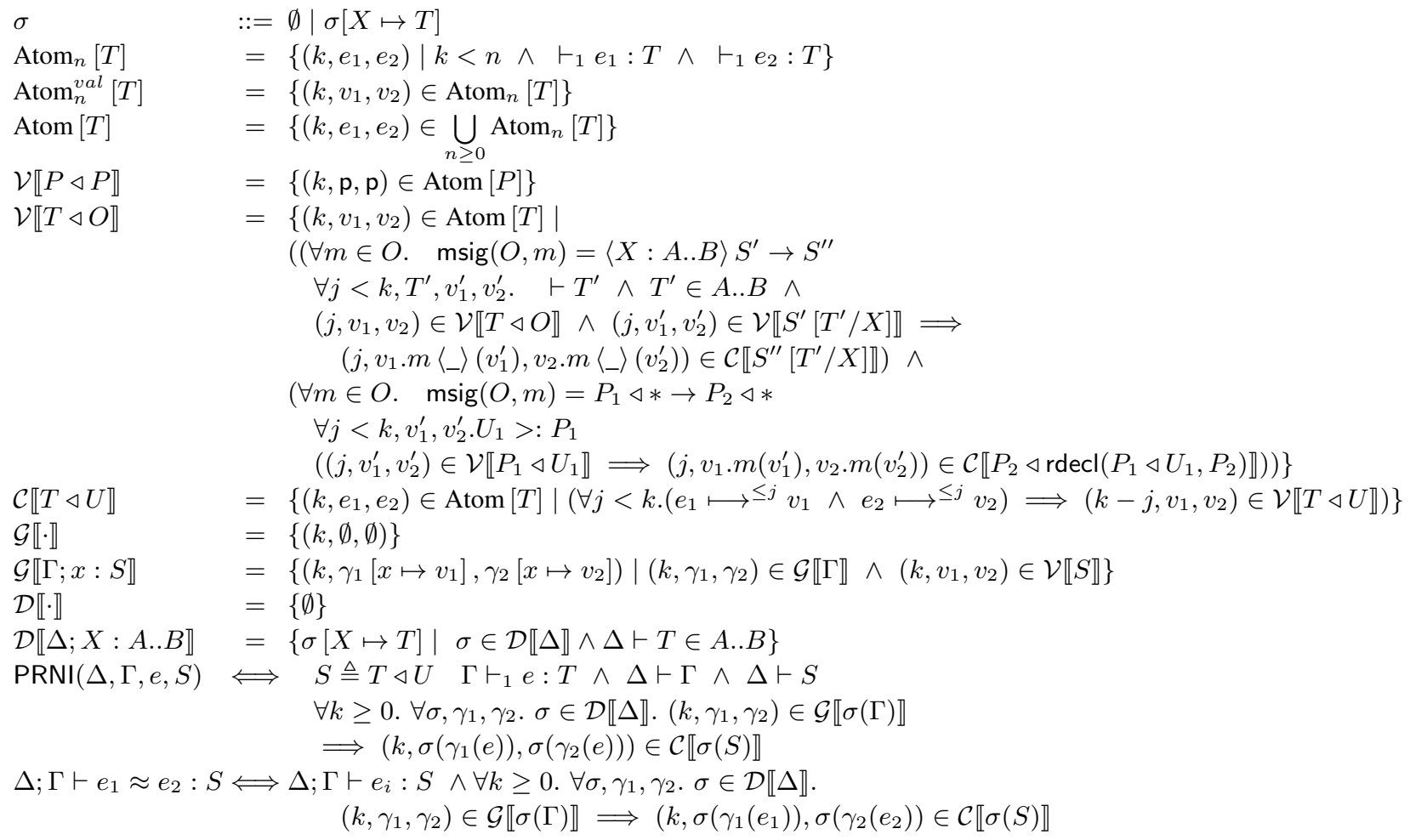

Fig. 26. $\mathrm{Ob}_{\mathrm{SEC}}^{\langle\rangle}$. Step-indexed logical relations for PRNI

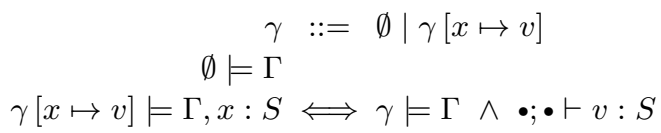

Fig. 27. $\mathrm{Ob}_{\mathrm{SEC}}^{\langle\rangle}$. PRNI. Auxiliary definition (used in proofs) 
B. Auxiliary Lemma: Simple typing

Lemma 8 (Well-type programs are simple well-typed).

If $\Delta ; \Gamma \vdash e: T \triangleleft U$ then $\Gamma \vdash_{1} e: T$

Lemma 9 (Security subtyping implies simple subtyping).

If $\Delta ; \Phi \vdash T^{\prime}<: T$ then $\Phi \vdash_{\text {sf }} T^{\prime}<: T$

Lemma 10 (Value substitution preserves simple typing). If $\Gamma \vdash_{1} e: T$ and $\gamma=\Gamma$ then $\vdash_{1} \gamma(e): T$

Proof. By induction on $\Gamma$. 


\section{Auxiliary Lemma: Logical relation}

1) Atom subtyping:

Lemma 11 (Atom subtyping).

If $\left(k, v_{1}, v_{2}\right) \in$ Atom $\left[T^{\prime}\right]$ and $\bullet ; \bullet \vdash T^{\prime}<: T$

then $\left(k, v_{1}, v_{2}\right) \in$ Atom $[T]$

Proof. Proof obligations:

$\vdash_{1} e_{i}: T(i \in\{1,2\})$. Apply rule (T1Sub). Note that $\bullet ; \bullet \vdash T^{\prime}<: T \Rightarrow \bullet \vdash_{\mathrm{sf}} T^{\prime}<: T$ (Lemma 9 , Security subtyping implies simple subtyping

2) Atom reduction:

Lemma 12 (Atom reduction).

Let $e_{1} \longmapsto^{*} e_{1}^{\prime}$ and $e_{2} \longmapsto^{*} e_{2}^{\prime}$

Let $\left(k, e_{1}, e_{2}\right) \in$ Atom $[T]$

Then $\left(k, e_{1}^{\prime}, e_{2}^{\prime}\right) \in$ Atom $[T]$

Proof. The proof is straightforward. Each subgoal follows by induction on the typing derivation of $e_{i}(i \in\{1,2\})$.

3) Type substitution preserves subtyping:

Lemma 13 (Type substitution preserves subtyping).

Let $\sigma \in \mathcal{D} \llbracket \Delta \rrbracket$

If $\Delta, \bullet \vdash S<: S^{\prime}$ then $\bullet ; \bullet \vdash \sigma(S)<: \sigma\left(S^{\prime}\right)$

If $\Delta, \bullet \vdash T<: T^{\prime}$ then $\bullet ; \bullet \vdash \sigma(T)<: \sigma\left(T^{\prime}\right)$

4) Type substitution preserves interval subtyping:

Lemma 14 (Type substitution preserves interval subtyping).

Let $\sigma \in \mathcal{D} \llbracket \Delta \rrbracket$ and $\Delta \vdash U, \Delta \vdash A, \Delta \vdash B$

If $\Delta \vdash U \in A . . B$

then $\sigma(U) \in \sigma(A) . . \sigma(B)$

Proof. Apply Lemma 13 (Type substitution preserves subtyping for each subgoal.

5) Interval subtyping expansion:

Lemma 15 (Interval subtyping expansion).

Let $\sigma \in \mathcal{D} \llbracket \Delta \rrbracket$ and $\bullet \vdash U, \Delta \vdash A, \Delta \vdash B$

If $\bullet \vdash U \in \sigma(A) . . \sigma(B)$

then $\Delta \vdash U \in A$...B

6) Downward closed/Monotonicity:

Lemma 16 (Downward closed/Monotonicity).

Let $\bullet \vdash S$

If $\left(k, v_{1}, v_{2}\right) \in \mathcal{V} \llbracket S \rrbracket$ and $j \leq k$

then $\left(j, v_{1}, v_{2}\right) \in \mathcal{V} \llbracket S \rrbracket$

Proof. The proof is by induction on $S$. All valid cases boil down to $P \triangleleft P$ and $T \triangleleft O$

Case $(\mathrm{S}=P \triangleleft P)$. This is direct from the definition of $\mathcal{V} \llbracket P \triangleleft P \rrbracket$

Case $(\mathrm{S}=T \triangleleft O)$.

Proof obligations:

1) $\left(j, v_{1}, v_{2}\right) \in$ Atom $[T]$. This follows directly from $\left(k, v_{1}, v_{2}\right) \in \mathcal{V} \llbracket S \rrbracket$

2) Assuming arbitrary $m, j^{\prime}, T^{\prime}, v_{1}^{\prime}, v_{2}^{\prime}$ such as:

- $m \in O \wedge \operatorname{msig}(\bullet ; O, m)=\langle X: A . . B\rangle S_{1} \rightarrow S_{2}$

- $j^{\prime}<j$

- $\vdash T^{\prime} \wedge T^{\prime} \in A$...B

- $\left(j^{\prime}, v_{1}, v_{2}\right) \in \mathcal{V} \llbracket S \rrbracket$

- $\left(j^{\prime}, v_{1}^{\prime}, v_{2}^{\prime}\right) \in \mathcal{V} \llbracket S_{1}\left[X / T^{\prime}\right] \rrbracket$ 
Show:

$$
\left(j, v_{1} \cdot m\left\langle T^{\prime}\right\rangle\left(v_{1}^{\prime}\right), v_{2} \cdot m\left\langle T^{\prime}\right\rangle\left(v_{2}^{\prime}\right)\right) \in \mathcal{C} \llbracket S^{\prime \prime}\left[X / T^{\prime}\right] \rrbracket
$$

Instantiate the first conjunct of $\left(k, v_{1}, v_{2}\right) \in \mathcal{V} \llbracket S \rrbracket$ with $m, j^{\prime}, T^{\prime}, v_{1}^{\prime}, v_{2}^{\prime}$. Note that:

- $m \in O \wedge \operatorname{msig}(\bullet ; O, m)=\langle X: A . . B\rangle S_{1} \rightarrow S_{2}$

- $j^{\prime}<k$. It follows from $j^{\prime}<j \leq k$

- $\vdash T^{\prime} \wedge T^{\prime} \in A$...B

- $\left(j^{\prime}, v_{1}, v_{2}\right) \in \mathcal{V} \llbracket S \rrbracket$

- $\left(j^{\prime}, v_{1}^{\prime}, v_{2}^{\prime}\right) \in \mathcal{V} \llbracket S_{1}\left[X / T^{\prime}\right] \rrbracket$

Hence $\left(j, v_{1} . m\left\langle T^{\prime}\right\rangle\left(v_{1}^{\prime}\right), v_{2} . m\left\langle T^{\prime}\right\rangle\left(v_{2}^{\prime}\right)\right) \in \mathcal{C} \llbracket S^{\prime \prime}\left[X / T^{\prime}\right] \rrbracket$

3) Assuming arbitrary $m, j, v_{1}^{\prime}, v_{2}^{\prime}$ such as:

- $m \in O \quad \operatorname{msig}(\bullet, O, m)=P_{1} \triangleleft * \rightarrow P_{2} \triangleleft *$

- $\left(j, v_{1}^{\prime}, v_{2}^{\prime}\right) \in \mathcal{V} \llbracket P_{1} \triangleleft U_{1} \rrbracket$

Show:

$$
\left(j^{\prime}, v_{1} . m\left(v_{1}^{\prime}\right), v_{2} . m\left(v_{2}^{\prime}\right)\right) \in \mathcal{C} \llbracket P_{2} \triangleleft \operatorname{rdecl}\left(P_{1} \triangleleft U_{1}, P_{2}\right) \rrbracket
$$

Instantiate the second conjunct of $\left(k, v_{1}, v_{2}\right) \in \mathcal{V} \llbracket S \rrbracket$ with $m, j^{\prime}, v_{1}^{\prime}, v_{2}^{\prime}$. Note that:

- $m \in O \quad \operatorname{msig}(\bullet, O, m)=P_{1} \triangleleft * \rightarrow P_{2} \triangleleft *$

- $j^{\prime}<k$. It follows from $j^{\prime}<j \leq k$

- $\left(j, v_{1}^{\prime}, v_{2}^{\prime}\right) \in \mathcal{V} \llbracket P_{1} \triangleleft U_{1} \rrbracket$

Hence $\left(j^{\prime}, v_{1} . m\left(v_{1}^{\prime}\right), v_{2} . m\left(v_{2}^{\prime}\right)\right) \in \mathcal{C} \llbracket P_{2} \triangleleft \operatorname{rdecl}\left(P_{1} \triangleleft U_{1}, P_{2}\right) \rrbracket$

7) Syntactic equivalences implies semantic equivalence:

Lemma 5 (Equal values are logically related).

$\forall k \geq 0, \mathrm{p}, P, O$.

$$
\vdash_{1} \mathrm{p}: O \wedge P<: O \Longrightarrow(k, \mathrm{p}, \mathrm{p}) \in \mathcal{V} \llbracket P \triangleleft O \rrbracket
$$

Proof. Assuming arbitrary $m, j, v_{1}^{\prime}, v_{2}^{\prime}$ such as:

- $m \in O \quad \operatorname{msig}(\bullet, O, m)=P_{1} \triangleleft * \rightarrow P_{2} \triangleleft *$

- $\left(j, v_{1}^{\prime}, v_{2}^{\prime}\right) \in \mathcal{V} \llbracket P_{1} \triangleleft U_{1} \rrbracket$

Show:

$$
\left(j, v_{1} \cdot m\left(v_{1}^{\prime}\right), v_{2} \cdot m\left(v_{2}^{\prime}\right)\right) \in \mathcal{C} \llbracket P_{2} \triangleleft \operatorname{rdecl}\left(P_{1} \triangleleft U_{1}, P_{2}\right) \rrbracket
$$

Case $\left(U_{1}=P_{1}\right)$. The $v_{1}^{\prime}=v_{2}^{\prime}=\mathrm{p}^{\prime}$ and we have to show

$$
\begin{gathered}
\left(j, \text { p. } m\left(\mathbf{p}^{\prime}\right), \text { p. } m\left(\mathbf{p}^{\prime}\right)\right) \in \mathcal{C} \llbracket P_{2} \triangleleft P_{2} \rrbracket \\
\equiv\left(j, \theta\left(m, \text { p, } \mathbf{p}^{\prime}\right), \theta\left(m, \mathrm{p}, \mathbf{p}^{\prime}\right)\right) \in \mathcal{C} \llbracket P_{2} \triangleleft P_{2} \rrbracket
\end{gathered}
$$

which follows from the assumption that $\theta$ is partial function that respects that signatures of meths $(P)$.

Case $\left(U_{1} \neq P_{1}\right)$. Then we have to show

$$
\left(j, \text { p. } m\left(v_{1}^{\prime}\right), \text { p. } m\left(v_{2}^{\prime}\right)\right) \in \mathcal{C} \llbracket P_{2} \triangleleft \top \rrbracket
$$

which trivially follows by using Lemma 21 Well-typed terms are related at top.

\section{8) PER Subtyping:}

Lemma 17 (PER Subtyping).

Let $\bullet \vdash S, \bullet \vdash S^{\prime}$ and $\bullet \bullet \vdash S^{\prime}<: S$

(1) If $\left(k, v_{1}, v_{2}\right) \in \mathcal{V} \llbracket S^{\prime} \rrbracket$ then $\left(k, v_{1}, v_{2}\right) \in \mathcal{V} \llbracket S \rrbracket$

(2) If $\left(k, e_{1}, e_{2}\right) \in \mathcal{C} \llbracket S^{\prime} \rrbracket$ then $\left(k, e_{1}, e_{2}\right) \in \mathcal{C} \llbracket S \rrbracket$

Proof. We proof the statements (1) and (2) simultaneously.

Induction on $k$ and then nested induction on $S$.

We focus on $k>0$ (the case $k=0$ is trivial).

Statement (1): 
All valid cases for $S$ boils down to $P \triangleleft P$ and $T \triangleleft O$.

Case $(\mathrm{S}=P \triangleleft P)$.

Proof obligations:

1) $\left(k, v_{1}, v_{2}\right) \in$ Atom $[P]$. Apply Lemma 11 Atom subtyping

2) $v_{1}=v_{2}=b$. From the third hypothesis we know that $S^{\prime}=P \triangleleft P$ and for the second one we know that $v_{1}=v_{2}=b$

Case $(\mathrm{S}=T \triangleleft O)$.

Denote $S^{\prime}=T^{\prime} \triangleleft O^{\prime}$

Proof obligations:

1) $\left(k, v_{1}, v_{2}\right) \in$ Atom $[T]$. Apply Lemma 11 Atom subtyping

2) Assuming arbitrary $m, j<k, T^{\prime}, v_{1}^{\prime}, v_{2}^{\prime}$ such as:

- $m \in O \quad \operatorname{msig}(\bullet, O, m)=\langle X: A . . B\rangle S_{1} \rightarrow S_{2}$

- $\vdash T^{\prime} \wedge T^{\prime} \in A$...B

- $\left(j, v_{1}, v_{2}\right) \in \mathcal{V} \llbracket S \rrbracket$

- $\left(j, v_{1}^{\prime}, v_{2}^{\prime}\right) \in \mathcal{V} \llbracket S_{1}\left[X / T^{\prime}\right] \rrbracket$

Show:

$$
\left(j, o_{1} . m\left\langle T^{\prime}\right\rangle\left(v_{1}^{\prime}\right), o_{2} \cdot m\left\langle T^{\prime}\right\rangle\left(v_{2}^{\prime}\right)\right) \in \mathcal{C} \llbracket S_{2}\left[X / T^{\prime} \rrbracket \rrbracket\right)
$$

Instantiate $\left(k, v_{1}, v_{2}\right) \in \mathcal{V} \llbracket T^{\prime} \triangleleft O^{\prime} \rrbracket$ with $m, j, T^{\prime}, v_{1}^{\prime}, v_{2}^{\prime}$. Note that:

- $m \in O^{\prime} \operatorname{msig}\left(\bullet, O^{\prime}, m\right)=\left\langle X: L^{\prime} . . U^{\prime}\right\rangle S_{1}^{\prime} \rightarrow S_{2}^{\prime}$

- $j<k$

- $\vdash T^{\prime} \wedge T^{\prime} \in A^{\prime} . . B^{\prime}$.

- $\left(j, v_{1}^{\prime}, v_{2}^{\prime}\right) \in \mathcal{V} \llbracket S_{1}^{\prime}\left[X / T^{\prime}\right] \rrbracket$. Apply the IH with $\left(j, v_{1}^{\prime}, v_{2}^{\prime}\right) \in \mathcal{V} \llbracket S_{1}\left[X / T^{\prime}\right] \rrbracket$ and $\bullet ; \bullet \vdash S_{1}\left[X / T^{\prime}\right]<: S_{1}^{\prime}\left[X / T^{\prime}\right]$

Hence, $\left.\left(j, o_{1} . m\left\langle T^{\prime}\right\rangle\left(v_{1}^{\prime}\right), o_{2} . m\left\langle T^{\prime}\right\rangle\left(v_{2}^{\prime}\right)\right) \in \mathcal{C} \llbracket S_{2}^{\prime \prime}\left[X / T^{\prime}\right] \rrbracket\right)$

Apply IH, statement (2) with $\bullet \cdot \vdash S_{2}^{\prime \prime}\left[X / T^{\prime}\right]<: S_{2}\left[X / T^{\prime}\right]$ to obtain $\left.\left(j, o_{1} \cdot m\left\langle T^{\prime}\right\rangle\left(v_{1}^{\prime}\right), o_{2} \cdot m\left\langle T^{\prime}\right\rangle\left(v_{2}^{\prime}\right)\right) \in \mathcal{C} \llbracket S_{2}\left[X / T^{\prime}\right] \rrbracket\right)$

3) Assuming arbitrary $m, j, v_{1}^{\prime}, v_{2}^{\prime}$ such as:

- $m \in O \quad \operatorname{msig}(\bullet, O, m)=P_{1} \triangleleft * \rightarrow P_{2} \triangleleft *$

- $\left(j, v_{1}^{\prime}, v_{2}^{\prime}\right) \in \mathcal{V} \llbracket P_{1} \triangleleft U_{1} \rrbracket$

Show:

$$
\left(j, v_{1} \cdot m\left(v_{1}^{\prime}\right), v_{2} \cdot m\left(v_{2}^{\prime}\right)\right) \in \mathcal{C} \llbracket P_{2} \triangleleft \operatorname{rdecl}\left(P_{1} \triangleleft U_{1}, P_{2}\right) \rrbracket
$$

Do a case analysis on $S^{\prime}$. Each case reduces to $S^{\prime}=T^{\prime} \triangleleft O^{\prime}$ or $S^{\prime}=P^{\prime} \triangleleft P^{\prime}$

Case $\left(S^{\prime}=T^{\prime} \triangleleft O^{\prime}\right)$. Instantiate $\left(k, v_{1}, v_{2}\right) \in \mathcal{V} \llbracket T^{\prime} \triangleleft O^{\prime} \rrbracket$ with $m, j, v_{1}^{\prime}, v_{2}^{\prime}$. Note that:

- $m \in O \quad \operatorname{msig}(\bullet, O, m)=P_{1} \triangleleft * \rightarrow P_{2} \triangleleft *$. Recall, that there is no subtyping rules between primitive types.

- $\left(j, v_{1}^{\prime}, v_{2}^{\prime}\right) \in \mathcal{V} \llbracket P_{1} \triangleleft U_{1} \rrbracket$

Hence $\left(j, v_{1} \cdot m\left(v_{1}^{\prime}\right), v_{2} . m\left(v_{2}^{\prime}\right)\right) \in \mathcal{C} \llbracket P_{2} \triangleleft \operatorname{rdecl}\left(P_{1} \triangleleft U_{1}, P_{2}\right) \rrbracket$

Case $\left(S^{\prime}=P^{\prime} \triangleleft P^{\prime}\right)$.

It means that $v_{1}=v_{2}=b$. Apply Lemma 5 Equal values are logically related with $b, P^{\prime}$ and $O$. Note that $\vdash_{1} b: P^{\prime}$ and $P^{\prime}<: O$.

Statement (2):

Denote $S \triangleq T \triangleleft U$ and $S^{\prime} \triangleq T^{\prime} \triangleleft U^{\prime}$

Proof obligations:

1) $\left(k, e_{1}, e_{2}\right) \in \operatorname{Atom}[T]$. Apply Lemma 11 Atom subtyping with $\left(k, e_{1}, e_{2}\right) \in \operatorname{Atom}\left[T^{\prime}\right]\left(\left(k, e_{1}, e_{2}\right) \in \mathcal{C} \llbracket S^{\prime} \rrbracket\right)$ and $\bullet ; \bullet T^{\prime}<: T$

2) Assuming arbitrary $j<k, v_{1}, v_{2}$ such as $j<k$ :

- $e_{1} \longmapsto{ }^{j} v_{1}$

- $e_{2} \longmapsto{ }^{j} v_{2}$

Show:

$$
\left(j, v_{1}, v_{2}\right) \in \mathcal{V} \llbracket S \rrbracket
$$

Instantiate $\left(k, e_{1}, e_{2}\right) \in \mathcal{C} \llbracket S^{\prime} \rrbracket$ with $j, v_{1}, v_{2}$ to obtain: $\left(k, v_{1}, v_{2}\right) \in \mathcal{V} \llbracket S^{\prime} \rrbracket$.

Apply the IH, statement (1) with $\left(k, v_{1}, v_{2}\right) \in \mathcal{V} \llbracket S^{\prime} \rrbracket$ and $\bullet ; \bullet \vdash S^{\prime}<: S$ to obtain:

$\left(k, v_{1}, v_{2}\right) \in \mathcal{V} \llbracket S \rrbracket$ 
9) Anti reduction:

Lemma 18 (Anti reduction).

Let $S \triangleq T \triangleleft U$

Let $\left(j, e_{1}, e_{2}\right) \in$ Atom $[T]$

Let $j^{\prime} \leq j$ and $j \leq j^{\prime}+k$

Let $e_{1} \longmapsto \leq k \quad e_{1}^{\prime}$ and $e_{2} \longmapsto \leq k e_{2}^{\prime}$

Let $\left(j^{\prime}, e_{1}^{\prime}, e_{2}^{\prime}\right) \in \mathcal{C} \llbracket S \rrbracket$

Then $\left(j, e_{1}, e_{2}\right) \in \mathcal{C} \llbracket S \rrbracket$

Proof. Denote $S \triangleq T \triangleleft U$.

Proof obligations:

1) $\left(j, e_{1}, e_{2}\right) \in$ Atom $[T]$. Apply Lemma 12 Atom reduction with $\left(j^{\prime}, e_{1}^{\prime}, e_{2}^{\prime}\right) \in$ Atom $[T]$ which follows from $\left(j^{\prime}, e_{1}^{\prime}, e_{2}^{\prime}\right) \in$ $\mathcal{C} \llbracket S \rrbracket$

2) Assuming $j_{1}<j, v_{1}, v_{2}$ such as:

- $e_{1} \longmapsto \leq j_{1} v_{1}$

- $e_{1} \longmapsto \leq j_{1} \quad v_{2}$

\section{Show:}

We have that:

$$
\left(j-j_{1}, v_{1}, v_{2}\right) \in \mathcal{V} \llbracket S \rrbracket
$$

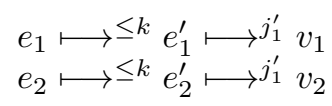

where $j_{1}^{\prime}<j^{\prime}$.

Instantiate $\left(j^{\prime}, e_{1}^{\prime}, e_{2}^{\prime}\right) \in \mathcal{C} \llbracket S \rrbracket$ with $j_{1}^{\prime}$. Note that $j_{1}^{\prime}<j^{\prime}$.

Hence, $\left(j^{\prime}-j_{1}^{\prime}, v_{1}, v_{2}\right) \in \mathcal{V} \llbracket S \rrbracket$.

Apply Lemma 16 Downward closed/Monotonicity with $j-j_{1} \leq j-\left(k+j_{1}^{\prime}\right) \leq j^{\prime}-j_{1}^{\prime}\left(j-k \leq j^{\prime}\right)$ to obtain: $\left(j-j_{1}, v_{1}, v_{2}\right) \in \mathcal{V} \llbracket S \rrbracket$

10) Monadic bind:

Lemma 19 (Monadic bind).

If $\left(k, e_{1}, e_{2}\right) \in \mathcal{C} \llbracket S \rrbracket$

and $\forall j \leq k . \forall v_{1}, v_{2} .\left(j, v_{1}, v_{2}\right) \in \mathcal{V} \llbracket S \rrbracket \Longrightarrow\left(j, E\left[v_{1}\right], E\left[v_{2}\right]\right) \in \mathcal{C} \llbracket S^{\prime} \rrbracket$

then $\left(k, E\left[e_{1}\right], E\left[e_{2}\right]\right) \in \mathcal{C} \llbracket S^{\prime} \rrbracket$

Proof. Let us assume that $e_{2} \longmapsto \leq j^{\prime} v_{1}^{\prime}$ and $e_{2} \longmapsto \leq j^{\prime} v_{2}^{\prime}$ where $j^{\prime} \leq k$ (in other case the lemma vacuously holds)

Instantiate $\left(k, e_{1}, e_{2}\right) \in \mathcal{C} \llbracket S \rrbracket$ with $j^{\prime}, v_{1}^{\prime}, v_{2}^{\prime}$.

Hence, $\left(k-j^{\prime}, v_{1}^{\prime}, v_{2}^{\prime}\right) \in \mathcal{V} \llbracket S^{\prime} \rrbracket$

By the dynamic semantics we have know that:

$$
\begin{aligned}
& E\left[e_{1}\right] \longmapsto \leq j^{\prime} E\left[v_{1}^{\prime}\right] \\
& E\left[e_{2}\right] \longmapsto \leq j^{\prime} E\left[v_{2}^{\prime}\right]
\end{aligned}
$$

Instantiate the second premise with $k-j^{\prime}, v_{1}^{\prime}, v_{2}^{\prime}$. Note that $k-j^{\prime} \leq k$ and $\left(k-j^{\prime}, v_{1}^{\prime}, v_{2}^{\prime}\right) \in \mathcal{V} \llbracket S \rrbracket$.

Hence, $\left(k-j^{\prime}, E\left[v_{1}^{\prime}\right], E\left[v_{2}^{\prime}\right]\right) \in \mathcal{C} \llbracket S^{\prime} \rrbracket$

Instantiate Lemma 18 Anti reduction. Note that:

- $k-j^{\prime} \leq k$

- $k \leq k-j^{\prime}+j^{\prime}$

- $E\left[e_{1}\right] \longmapsto \leq j^{\prime} E\left[v_{1}^{\prime}\right]$

- $E\left[e_{2}\right] \longmapsto \leq j^{\prime} E\left[v_{2}^{\prime}\right]$

- $\left(k-j^{\prime}, E\left[v_{1}^{\prime}\right], E\left[v_{2}^{\prime}\right]\right) \in \mathcal{C} \llbracket S^{\prime} \rrbracket$

Hence, $\left(k, E\left[e_{1}\right], E\left[e_{2}\right]\right) \in \mathcal{C} \llbracket S^{\prime} \rrbracket$ 
11) Substitutions preserve simple typing:

Lemma 20 (Substitutions preserve simple typing).

Let $\Delta, \Gamma \vdash e_{1}: T_{1} \triangleleft U_{1}$ and $\Delta, \Gamma \vdash e_{2}: T_{1} \triangleleft U_{1}$

Let $\sigma \in \mathcal{D} \llbracket \Delta \rrbracket$ and $\left(k, \gamma_{1}, \gamma_{2}\right) \in \mathcal{G} \llbracket \sigma(\Gamma) \rrbracket$

Then $\left(k, \sigma\left(\gamma_{1}\left(e_{1}\right)\right), \sigma\left(\gamma_{2}\left(e_{2}\right)\right)\right) \in \operatorname{Atom}\left[\sigma\left(T_{1}\right)\right]$

Proof. The proof is straightforward. Then the goal is equivalent to show: $\left(k, \gamma_{1}\left(e_{1}\right), \gamma_{2}\left(e_{2}\right)\right) \in$ Atom $\left[\sigma\left(T_{1}\right)\right]$ (because type variable are not taken into account by the simple type system).

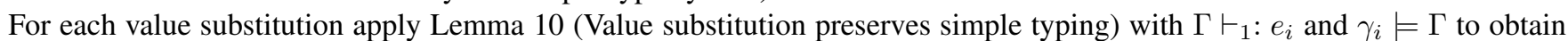
$\vdash_{1} \gamma_{i}\left(e_{i}\right): \sigma\left(T_{1}\right)$

12) Well-typed terms are related at top:

Lemma 21 (Well-typed terms are related at top).

Let $\sigma \in \mathcal{D} \llbracket \Delta \rrbracket$ and $\left(k, \gamma_{1}, \gamma_{2}\right) \in \mathcal{G} \llbracket \sigma(\Gamma) \rrbracket$

Let $\Delta ; \Gamma \vdash e_{1}: T \triangleleft \top$

Let $\Delta ; \Gamma \vdash e_{2}: T \triangleleft \top$

Then $\left(k, \sigma\left(\gamma_{1}\left(e_{1}\right)\right), \sigma\left(\gamma_{2}\left(e_{2}\right)\right)\right) \in \mathcal{C} \llbracket \sigma(T) \triangleleft \top \rrbracket$

Proof. Proof obligations

1) $\left(k, \sigma\left(\gamma_{1}\left(e_{1}\right)\right), \sigma\left(\gamma_{1}\left(e_{2}\right)\right)\right) \in$ Atom $[\sigma(U)]$. It follows from Lemma 20 Substitutions preserve simple typing.

2) Assuming $j<k, v_{1}, v_{2}$ such as:

- $\sigma\left(\gamma_{1}\left(e_{1}\right)\right) \longmapsto \leq j v_{1}$

- $\sigma\left(\gamma_{2}\left(e_{2}\right)\right) \longmapsto \leq j v_{2}$

Show

Which is equivalent to show

$$
\left(k-j, v_{1}, v_{2}\right) \in \mathcal{V} \llbracket \sigma(T) \triangleleft \top \rrbracket
$$

$$
\left(k-j, v_{1}, v_{2}\right) \in \operatorname{Atom}[\sigma(T)]
$$

Apply Lemma 12 Atom reduction with $\left(k, \sigma\left(\gamma_{1}\left(e_{1}\right)\right), \sigma\left(\gamma_{1}\left(e_{2}\right)\right)\right) \in$ Atom $[\sigma(T)], v_{1}$ and $v_{2}$ to obtain $\left(k-j, v_{1}, v_{2}\right) \in$ Atom $[\sigma(T)]$

13) Related values are related terms:

Lemma 22 (Related values are related terms). If $\left(k, v_{1}, v_{2}\right) \in \mathcal{V} \llbracket S \rrbracket$ then $\left(k, v_{1}, v_{2}\right) \in \mathcal{C} \llbracket S \rrbracket$

Proof. The proof trivially follows. 


\section{Proof of Fundamental Property}

1) Pre-compatibility: Method Invocation:

Lemma 23 (Pre-compatibility: Method Invocation).

Let $\sigma \in \mathcal{D} \llbracket \Delta \rrbracket, \Delta \vdash T_{1} \triangleleft U_{1}$

Let $k^{\prime \prime} \leq k^{\prime} \leq k$

Let $\left(k^{\prime}, v_{1}, v_{2}\right) \in \mathcal{V} \llbracket \sigma\left(T_{1} \triangleleft U_{1}\right) \rrbracket$

Let $\Delta \vdash m \in U_{1}$ and $\operatorname{msig}\left(\Delta ; U_{1}, m\right)=\langle X: A . . B\rangle S_{2} \rightarrow S$

Let $\Delta \vdash U^{\prime} \wedge \Delta \vdash U^{\prime} \in A$...B

Let $\left(k, v_{1}^{\prime \prime}, v_{2}^{\prime \prime}\right) \in \mathcal{V} \llbracket \sigma\left(S_{2}\left[U^{\prime} / X\right]\right) \rrbracket$

then $\left(k^{\prime \prime}, v_{1} . m\left\langle\sigma\left(U^{\prime}\right)\right\rangle\left(v_{1}^{\prime \prime}\right), v_{2} . m\left\langle\sigma\left(U^{\prime}\right)\right\rangle\left(v_{2}^{\prime \prime}\right)\right) \in \mathcal{C} \llbracket \sigma\left(S\left[U^{\prime} / X\right]\right) \rrbracket$

Proof. Instantiate $\left(k^{\prime}, v_{1}, v_{2}\right) \in \mathcal{V} \llbracket \sigma\left(T_{1} \triangleleft U_{1}\right) \rrbracket$ with: $m, k^{\prime \prime}, \sigma\left(U^{\prime}\right), v_{1}^{\prime \prime}, v_{2}^{\prime \prime}$. Note that:

- $m \in \sigma\left(T_{1}\right)$. It follows from $\Delta \vdash m \in U_{1}, \sigma \in \mathcal{D} \llbracket \Delta \rrbracket$ and $\Delta \vdash T_{1} \triangleleft U_{1}$. Then $\operatorname{msig}\left(\bullet, \sigma\left(T_{1}\right), m\right)=\langle X: \sigma(A) . . \sigma(B)\rangle \sigma\left(S_{2}\right) \rightarrow \sigma(S)$.

- $k^{\prime \prime}<k$ which follows directly from hypothesis.

- $\vdash \sigma\left(U^{\prime}\right)$ which is direct from $\Delta \vdash U^{\prime}$ and $\rho \in \mathcal{D} \llbracket \Delta \rrbracket$.

- $\sigma\left(U^{\prime}\right) \in \sigma(A) . . \sigma(B)$. Apply Lemma 14 (Type substitution preserves interval subtyping) with $\sigma \in \mathcal{D} \llbracket \Delta \rrbracket$ and $\Delta \vdash U^{\prime} \in$ $A . . B$

- $\left(k^{\prime \prime}, v_{1}^{\prime \prime}, v_{2}^{\prime \prime}\right) \in \mathcal{V} \llbracket \sigma\left(S_{2}\right)\left[\sigma\left(U^{\prime}\right) / X\right] \rrbracket$. It follows from

- $\left(k, v_{1}^{\prime \prime}, v_{2}^{\prime \prime}\right) \in \mathcal{V} \llbracket \sigma\left(S_{2}\right)\left[\sigma\left(U^{\prime}\right) / X\right] \rrbracket$. Note that $\sigma\left(S_{2}\left[U^{\prime} / X\right]\right)=\sigma\left(S_{2}\right)\left[\sigma\left(U^{\prime}\right) / X\right]$ and $\left(k, v_{1}^{\prime \prime}, v_{2}^{\prime \prime}\right) \in \mathcal{V} \llbracket \sigma\left(S_{2}\left[U^{\prime} / X\right]\right) \rrbracket$ is given in hypothesis

- Apply Lemma 16 (Downward closed/Monotonicity) with $\left(k, v_{1}^{\prime \prime}, v_{2}^{\prime \prime}\right) \in \mathcal{V} \llbracket \sigma\left(S_{2}\right)\left[\sigma\left(U^{\prime}\right) / X\right] \rrbracket$ and $k^{\prime \prime} \leq k$ we obtain $\left(k^{\prime \prime}, v_{1}^{\prime \prime}, v_{2}^{\prime \prime}\right) \in \mathcal{V} \llbracket \sigma\left(S_{2}\right)\left[\sigma\left(U^{\prime}\right) / X\right] \rrbracket$.

Hence, $\left(k^{\prime \prime}, v_{1} . m\left\langle\sigma\left(U^{\prime}\right)\right\rangle\left(v_{1}^{\prime \prime}\right), v_{2} . m\left\langle\sigma\left(U^{\prime}\right)\right\rangle\left(v_{2}^{\prime \prime}\right)\right) \in \mathcal{C} \llbracket \sigma(S)\left[\sigma\left(U^{\prime}\right) / X\right] \rrbracket$

Note $\sigma\left(S\left[U^{\prime} / X\right]\right)=\sigma(S)\left[\sigma\left(U^{\prime}\right) / X\right]$.

Hence, $\left(k^{\prime \prime}, v_{1} . m\left\langle\sigma\left(U^{\prime}\right)\right\rangle\left(v_{1}^{\prime \prime}\right), v_{2} . m\left\langle\sigma\left(U^{\prime}\right)\right\rangle\left(v_{2}^{\prime \prime}\right)\right) \in \mathcal{C} \llbracket \sigma\left(S\left[U^{\prime} / X\right]\right) \rrbracket$

2) Compatibility-Var:

Lemma $24\left(\mathrm{Ob}_{\mathrm{SEC}}^{\langle\rangle}\right.$Compatibility-Var).

$\Delta ; \Gamma \vdash x \approx x: \Gamma(x)$

Proof. First, let us denote $S \triangleq \Gamma(x)$.

Proof obligations:

1) $\Delta ; \Gamma \vdash x: S$ which is direct.

2) Assuming arbitrary $k, \sigma, \gamma_{1}, \gamma_{2}$ such as: $k \geq 0, \sigma \in \mathcal{D} \llbracket \Delta \rrbracket, \gamma_{1}, \gamma_{2}, \quad\left(k, \gamma_{1}, \gamma_{2}\right) \in \mathcal{G} \llbracket \rho(\Gamma) \rrbracket$

Show

$$
\begin{aligned}
& \left(k, \sigma\left(\gamma_{1}(x)\right), \sigma\left(\gamma_{2}(x)\right)\right) \in \mathcal{C} \llbracket \sigma(S) \rrbracket \\
& \quad \equiv\left(k, \gamma_{1}(x), \gamma_{2}(x)\right) \in \mathcal{C} \llbracket \sigma(S) \rrbracket
\end{aligned}
$$

From $\left(k, \gamma_{1}, \gamma_{2}\right) \in \mathcal{G} \llbracket \sigma(\Gamma) \rrbracket$ we know that exists $v_{1}, v_{2}$ such as:

- $\gamma_{1}(x)=v_{1}$

- $\gamma_{2}(x)=v_{2}$

- $\left(k, v_{1}, v_{2}\right) \in \mathcal{V} \llbracket \sigma(S) \rrbracket$

Apply Lemma 22 Related values are related terms with $\left(k, \gamma_{1}(x), \gamma_{2}(x)\right) \in \mathcal{V} \llbracket \sigma(S) \rrbracket$ to obtain $\left(k, \gamma_{1}(x), \gamma_{2}(x)\right) \in \mathcal{C} \llbracket \sigma(S) \rrbracket$

3) Compatibility-Prim:

Lemma $25\left(\mathrm{Ob}_{\mathrm{SEC}}^{\langle\rangle}\right.$Compatibility-Prim).

Let $P=\Delta_{\mathrm{p}}(\mathrm{p})$.

Then $\Delta ; \Gamma \vdash b \approx b: P \triangleleft P$

Proof. Proof obligations:

1) $\Delta ; \Gamma \vdash b: P \triangleleft P$. Apply rule (TPrim)

2) Assuming arbitrary $k, \rho, \gamma_{1}, \gamma_{2}$ such as:

- $k \geq 0, \sigma \in \mathcal{D} \llbracket \Delta \rrbracket,\left(k, \gamma_{1}, \gamma_{2}\right) \in \mathcal{G} \llbracket \sigma(\Gamma) \rrbracket$ 


\section{Show:}

$$
\begin{gathered}
\left(k, \sigma\left(\gamma_{1}(b)\right), \sigma\left(\gamma_{2}(b)\right)\right) \in \mathcal{C} \llbracket \sigma(P \triangleleft P) \rrbracket \\
\equiv(k, b, b) \in \mathcal{C} \llbracket P \triangleleft P \rrbracket
\end{gathered}
$$

Apply Lemma 22 Related values are related terms with $(k, b, b) \in \mathcal{V} \llbracket P \triangleleft P \rrbracket$ to obtain:

$(k, b, b) \in \mathcal{C} \llbracket P \triangleleft P \rrbracket$

4) Compatibility Subsumption:

Lemma $26\left(\mathrm{Ob}_{\mathrm{SEC}}^{\langle\rangle}\right.$Compatibility-Subsumption).

Let $\Delta ; \Gamma \vdash e_{1} \approx e_{2}: S^{\prime}$. Let $\Delta ; \bullet \vdash S^{\prime}<: S$.

Then $\Delta ; \Gamma \vdash e_{1} \approx e_{2}: S$.

Proof. Proof obligations:

1) $\Delta ; \Gamma \vdash e_{1}: S$ and $\Delta ; \Gamma \vdash e_{2}: S$. Apply rule (TSub) with $\Delta ; \Gamma \vdash e_{i}: S^{\prime}$ (obtained from $\Delta ; \Gamma \vdash e_{1} \approx e_{2}: S^{\prime}$ ) and $\Delta ; \bullet \vdash S^{\prime}<: S$

2) Assuming arbitrary $k, \sigma, \gamma_{1}, \gamma_{2}$ such as :

- $k \geq 0, \rho \in \mathcal{D} \llbracket \Delta \rrbracket,\left(k, \gamma_{1}, \gamma_{2}\right) \in \mathcal{G} \llbracket \sigma(\Gamma) \rrbracket$

\section{Show:}

$$
\left(k, \sigma\left(\gamma_{1}\left(e_{1}\right)\right), \sigma\left(\gamma_{2}\left(e_{2}\right)\right)\right) \in \mathcal{C} \llbracket \rho(S) \rrbracket .
$$

Instantiate $\Delta ; \Gamma \vdash e_{1} \approx e_{2}: S^{\prime}$ with $k, \sigma, \gamma_{1}, \gamma_{2}$ to obtain:

$\left.\left(k, \sigma\left(\gamma_{1}\left(e_{1}\right)\right)\right), \sigma\left(\gamma_{2}\left(e_{2}\right)\right)\right) \in \mathcal{C} \llbracket \sigma\left(S^{\prime}\right) \rrbracket$.

Apply Lemma 17 PER Subtyping with $\bullet ; \bullet \vdash\left(S^{\prime}\right)<: \sigma(S)$. Note that:

- $\bullet \cdot \vdash \sigma\left(S^{\prime}\right)<: \sigma(S)$ follows from Lemma 13 (Type substitution preserves subtyping applied to $\sigma \in \mathcal{D} \llbracket \Delta \rrbracket$ and $\Delta ; \bullet \vdash S^{\prime}<: S$.

Hence, $\left(k, \sigma\left(\gamma_{1}\left(e_{1}\right)\right), \sigma\left(\gamma_{2}\left(e_{2}\right)\right)\right) \in \mathcal{C} \llbracket \rho(S) \rrbracket$.

\section{5) Compatibility Object:}

Lemma $27\left(\mathrm{Ob}_{\mathrm{SEC}}^{\langle\rangle}\right.$Compatibility-Object).

Let be $S \triangleq O \triangleleft U$

Let be $O \triangleq \boldsymbol{O b j}(\alpha) \cdot\left[\overline{m:\langle X: A . . B\rangle S^{\prime} \rightarrow S^{\prime \prime}}\right]$

Then:

$$
\begin{gathered}
\operatorname{msig}\left(\Delta, O, m_{i}\right)=\left\langle X: U_{l i} . U_{u i}\right\rangle S_{i}^{\prime} \rightarrow S_{i}^{\prime \prime} \\
\Delta, X: U_{l i} . U_{u i} ; \Gamma, z: S, x: S_{i}^{\prime} \vdash e_{i} \approx e_{i}^{\prime}: S_{i}^{\prime \prime} \\
\Delta ; \Gamma \vdash[z: S \Rightarrow \overline{m(x) e}] \approx\left[z: S \Rightarrow \overline{m(x) e^{\prime}}\right]: S
\end{gathered}
$$

Proof. Denote $o=[z: S \Rightarrow \overline{m(x) e}]$ and $o^{\prime}=\left[z: S \Rightarrow \overline{m(x) e^{\prime}}\right]$

Proof obligations:

1) $\Delta ; \Gamma \vdash o: S \wedge \Delta ; \Gamma \vdash o^{\prime}: S$. Apply rule (TObj)

2) Consider arbitrary $k, \sigma, \gamma_{1}, \gamma_{2}$ such as: $k \geq 0, \sigma \in \mathcal{D} \llbracket \Delta \rrbracket,\left(k, \gamma_{1}, \gamma_{2}\right) \in \mathcal{G} \llbracket \sigma(\Gamma) \rrbracket$

Show:

$$
\equiv\left(k,\left[z: \sigma(S) \Rightarrow \frac{\left(k, \sigma\left(\gamma_{1}(o)\right), \sigma\left(\gamma_{2}\left(o^{\prime}\right)\right)\right): \mathcal{C} \llbracket \sigma(S) \rrbracket}{\left.\left.m(x) \sigma\left(\gamma_{1}(e)\right)\right],\left[z: \sigma(S) \Rightarrow \overline{m(x) \sigma\left(\gamma_{2}\left(e^{\prime}\right)\right)}\right]\right): \mathcal{C} \llbracket \sigma(S) \rrbracket}\right.\right.
$$

Apply Lemma 22 (Related values are related terms) to transform the goal to

$$
\left(k,\left[z: \sigma(S) \Rightarrow \overline{m(x) \sigma\left(\gamma_{1}(e)\right)}\right],\left[z: \sigma(S) \Rightarrow \overline{m(x) \sigma\left(\gamma_{2}\left(e^{\prime}\right)\right)}\right]\right): \mathcal{V} \llbracket \sigma(S) \rrbracket
$$

Let us denote $o_{1}=\left[z: \sigma(S) \Rightarrow \overline{m(x) \sigma\left(\gamma_{1}(e)\right)}\right], o_{2}=\left[z: \sigma(S) \Rightarrow \overline{m(x) \sigma\left(\gamma_{2}\left(e^{\prime}\right)\right)}\right]$

By well-formedness of the type $S$ (Figure 19 we know that $\rho(U)$ is necesarily an object type (i.e. it is not a primitive type)

Proof of $\left(k, o_{1}, o_{2}\right) \in \mathcal{V} \llbracket \sigma(O) \triangleleft \sigma(U) \rrbracket$.

Sub goals

- $\left(k, o_{1}, o_{2}\right) \in \operatorname{Atom}[\sigma(O)]$. Apply Lemma 20 (Substitutions preserve simple typing) with $\Delta ; \Gamma \vdash o: S, \Delta ; \Gamma \vdash o^{\prime}: S$, $\sigma \in \mathcal{D} \llbracket \Delta \rrbracket$ and $\left(k, \gamma_{1}, \gamma_{2}\right) \in \mathcal{G} \llbracket \sigma(\Gamma) \rrbracket$ 
- Assuming arbitrary $m, j, T^{\prime}, v_{1}^{\prime}, v_{2}^{\prime}$ such as:

- $m \in \rho(U) \quad \operatorname{msig}(\bullet, \sigma(O), m)=\langle X: \sigma(A) . . \sigma(B)\rangle \sigma\left(S^{\prime}\right) \rightarrow \sigma\left(S^{\prime \prime}\right)$

$-j<k$

- $\vdash T^{\prime} \wedge T^{\prime} \in \sigma(A) . . \sigma(B)$

- $\left(j, o_{1}, o_{2}\right) \in \mathcal{V} \llbracket \sigma(S) \rrbracket$

- $\left(j, v_{1}^{\prime}, v_{2}^{\prime}\right) \in \mathcal{V} \llbracket \sigma\left(S^{\prime}\right) \rrbracket$

Show:

$$
\left(j, o_{1} \cdot m\left\langle T^{\prime}\right\rangle\left(v_{1}^{\prime}\right), o_{2} \cdot m\left\langle T^{\prime}\right\rangle\left(v_{2}^{\prime}\right)\right) \in \mathcal{C} \llbracket \sigma\left(S^{\prime \prime}\right) \rrbracket
$$

Denote methimpl $\left(o_{1}, m\right)=x . \sigma\left(\gamma_{1}(e)\right)$ and methimpl $\left(o_{1}, m\right)=x . \sigma\left(\gamma_{2}\left(e^{\prime}\right)\right)$

Then, the above goal rewrites to

$$
\begin{gathered}
\left(j, \sigma\left(\gamma_{1}(e)\right)\left[T^{\prime} / X\right]\left[o_{1} / z\right]\left[v_{1}^{\prime} / x\right], \sigma\left(\gamma_{2}\left(e^{\prime}\right)\right)\left[T^{\prime} / X\right]\left[o_{2} / z\right]\left[v_{2}^{\prime} / x\right]\right) \in \mathcal{C} \llbracket \sigma\left(S^{\prime \prime}\right) \rrbracket \\
\left(j, \sigma\left[X \mapsto T^{\prime}\right]\left(\gamma_{1}(e)\right)\left[o_{1} / z\right]\left[v_{1}^{\prime} / x\right], \sigma\left[X \mapsto T^{\prime}\right]\left(\gamma_{2}\left(e^{\prime}\right)\right)\left[o_{2} / z\right]\left[v_{2}^{\prime} / x\right]\right) \in \mathcal{C} \llbracket \sigma\left(S^{\prime \prime}\right) \rrbracket
\end{gathered}
$$

Instantiate the second conjunct of the IH $\Delta, X: A . . B ; \Gamma, z: S, x: S^{\prime} \vdash e \approx e^{\prime}: S^{\prime \prime}$ with

$j, \sigma^{\prime}=\sigma\left[X \mapsto T^{\prime}\right], \gamma_{1}^{\prime}=\gamma_{1}\left[z \mapsto o_{1}\right]\left[x \mapsto v_{1}^{\prime}\right], \gamma_{1}^{\prime}=\gamma_{2}\left[z \mapsto o_{2}\right]\left[x \mapsto v_{2}^{\prime}\right]$. Note that:

$-j \geq 0$

- $\sigma\left[X \mapsto T^{\prime}\right] \in \mathcal{D} \llbracket \Delta, X: A . . B \rrbracket$. It follows from:

* $\sigma \in \mathcal{D} \llbracket \Delta \rrbracket$. It follows from above.

$* \Delta \vdash T^{\prime} \in A . . B$. Apply Lemma 15 Interval subtyping expansion with $\sigma \in \mathcal{D} \llbracket \Delta \rrbracket$ and $\vdash T^{\prime}$ and $T^{\prime} \in$ $\sigma(A) . . \sigma(B)$

- $\left(j, \gamma_{1}^{\prime}, \gamma_{2}^{\prime}\right) \in \mathcal{G} \llbracket \sigma(\Gamma), z: \sigma(S), x: \sigma\left(S^{\prime}\right) \rrbracket$. It follows from:

$*\left(j, \gamma_{1}, \gamma_{2}\right) \in \mathcal{G} \llbracket \sigma(\Gamma) \rrbracket$. It follows from above.

$*\left(j, o_{1}, o_{2}\right) \in \mathcal{V} \llbracket \sigma(S) \rrbracket$. It follows from the above.

$*\left(j, v_{1}^{\prime}, v_{2}^{\prime}\right) \in \mathcal{V} \llbracket \sigma\left(S^{\prime}\right) \rrbracket$. It follows from the above.

Hence $\left(j, \sigma\left[X \mapsto T^{\prime}\right]\left(\gamma_{1}\left[z \mapsto o_{1}\right]\left[x \mapsto v_{2}^{\prime}\right](e)\right), \sigma\left[X \mapsto T^{\prime}\right]\left(\gamma_{2}\left[z \mapsto o_{2}\right]\left[x \mapsto v_{2}^{\prime}\right]\left(e^{\prime}\right)\right)\right) \in \mathcal{C} \llbracket \sigma\left(S^{\prime \prime}\right) \rrbracket$

Since $o_{1}, o_{2}, v_{1}^{\prime}, v_{2}^{\prime}$ are closed values with respect to type variables we can rewrite this as:

$$
\left(j, \sigma\left[X \mapsto T^{\prime}\right]\left(\gamma_{1}(e)\right)\left[o_{1} / z\right]\left[v_{1}^{\prime} / x\right], \sigma\left[X \mapsto T^{\prime}\right]\left(\gamma_{2}\left(e^{\prime}\right)\right)\left[o_{2} / z\right]\left[v_{2}^{\prime} / x\right]\right) \in \mathcal{C} \llbracket \sigma\left(S^{\prime \prime}\right) \rrbracket
$$


6) Compatibility Method Invocation Declassification:

Lemma 28 ( $\mathrm{Ob}_{\mathrm{SEC}}^{\langle\rangle}$Compatibility-Method-Invocation-Declassification).

Let $S_{1} \triangleq T_{1} \triangleleft U_{1}, S_{2} \triangleq T_{2} \triangleleft U_{2}$

Let $\Delta ; \Gamma \vdash e_{1} \approx e_{1}^{\prime}: T_{1} \triangleleft U_{1}$

Let $\Delta \vdash m \in U_{1}, \operatorname{msig}\left(\Delta ; U_{1}, m\right)=\langle X: A . . B\rangle S_{2} \rightarrow S$

Let $\Delta \vdash U^{\prime} \wedge \Delta \vdash U^{\prime} \in A$..B

Let $\Delta ; \Gamma \vdash e_{2} \approx e_{2}^{\prime}: S_{2}\left[U^{\prime} / X\right]$

Then $\Delta ; \Gamma \vdash e_{1} \cdot m\left\langle U^{\prime}\right\rangle\left(e_{2}\right) \approx e_{1}^{\prime} \cdot m\left\langle U^{\prime}\right\rangle\left(e_{2}^{\prime}\right): S\left[U^{\prime} / X\right]$

Proof. Let us denote $e=e_{1} \cdot m\left\langle U^{\prime}\right\rangle\left(e_{2}\right)$ and $e^{\prime}=e_{1}^{\prime} \cdot m\left\langle U^{\prime}\right\rangle\left(e_{2}^{\prime}\right)$

Proof obligations:

1) $\Delta ; \Gamma \vdash e: S\left[U^{\prime} / X\right]$ and $\Delta ; \Gamma \vdash e^{\prime}: S\left[U^{\prime} / X\right]$ which follow directly from the premises and the rule (TmD).

2) Assuming arbitrary $k, \sigma, \gamma_{1}, \gamma_{2}$ such as: $k \geq 0, \sigma \in \mathcal{D} \llbracket \Delta \rrbracket,\left(k, \gamma_{1}, \gamma_{2}\right) \in \mathcal{G} \llbracket \sigma(\Gamma) \rrbracket$

Show:

$$
\begin{gathered}
\left(k, \sigma\left(\gamma_{1}\left(e_{1} \cdot m\left\langle U^{\prime}\right\rangle\left(e_{2}\right)\right)\right), \sigma\left(\gamma_{2}\left(e_{1}^{\prime} \cdot m\left\langle U^{\prime}\right\rangle\left(e_{2}^{\prime}\right)\right)\right)\right) \in \mathcal{C} \llbracket \sigma\left(S\left[U^{\prime} / X\right]\right) \rrbracket \\
\equiv\left(k, \sigma\left(\gamma_{1}\left(e_{1}\right)\right) \cdot m\left\langle\sigma\left(U^{\prime}\right)\right\rangle\left(\sigma\left(\gamma_{1}\left(e_{2}\right)\right)\right), \sigma\left(\gamma_{2}\left(e_{1}^{\prime}\right)\right) \cdot m\left\langle\sigma\left(U^{\prime}\right)\right\rangle\left(\sigma\left(\gamma_{2}\left(e_{2}^{\prime}\right)\right)\right)\right) \in \mathcal{C} \llbracket \sigma\left(S\left[U^{\prime} / X\right]\right) \rrbracket
\end{gathered}
$$

(because $X \notin \operatorname{dom}(\sigma)$ )

Instantiate the hypothesis $\Delta ; \Gamma \vdash e_{1} \approx e_{1}^{\prime}: T_{1} \triangleleft U_{1}$ with $k, \sigma, \gamma_{1}, \gamma_{2}$, hence:

$\left(k, \sigma\left(\gamma_{1}\left(e_{1}\right)\right), \sigma\left(\gamma_{2}\left(e_{1}^{\prime}\right)\right)\right) \in \mathcal{C} \llbracket \sigma\left(T_{1} \triangleleft U_{1}\right) \rrbracket$

Let $k^{\prime} \leq k$ and let $\left(k^{\prime}, v_{1}, v_{2}\right) \in \mathcal{V} \llbracket \sigma\left(T_{1} \triangleleft U_{1}\right) \rrbracket$. By Lemma 19 (Monadic bind) we can rewrite the goal to show

$$
\left.\left(k^{\prime}, v_{1} \cdot m\left\langle\sigma\left(U^{\prime}\right)\right\rangle\left(\sigma\left(\gamma_{1}\left(e_{2}\right)\right)\right), v_{2} . m\left\langle\sigma\left(U^{\prime}\right)\right\rangle\left(\sigma\left(\gamma_{2}\left(e_{2}^{\prime}\right)\right)\right)\right)\right) \in \mathcal{C} \llbracket S\left[U^{\prime} / X\right] \rrbracket
$$

Instantiate the hypothesis $\Delta ; \Gamma \vdash e_{2} \approx e_{2}^{\prime}: S_{2}\left[U^{\prime} / X\right]$ with $k, \rho, \gamma_{1}, \gamma_{2}$, hence:

$\left(k, \sigma\left(\gamma_{1}\left(e_{2}\right)\right), \sigma\left(\gamma_{2}\left(e_{2}^{\prime}\right)\right) \in \mathcal{C} \llbracket \sigma\left(S_{2}\left[U^{\prime} / X\right]\right) \rrbracket\right.$

Let $k^{\prime \prime} \leq k^{\prime}$ and let $\left(k^{\prime \prime}, v_{1}^{\prime \prime}, v_{2}^{\prime \prime}\right) \in \mathcal{V} \llbracket \sigma\left(S_{2}\left[U^{\prime} / X\right]\right) \rrbracket$. By Lemma 19 Monadic bind we can rewrite the goal to show

$\left.\left(k^{\prime \prime}, v_{1} \cdot m\left\langle\sigma\left(U^{\prime}\right)\right\rangle\left(v_{1}^{\prime \prime}\right), v_{2} \cdot m\left\langle\sigma\left(U^{\prime}\right)\right\rangle\left(v_{2}^{\prime \prime}\right)\right)\right) \in \mathcal{C} \llbracket \sigma\left(S\left[U^{\prime} / X\right]\right) \rrbracket$

Then, we apply the Lemma 23 (Pre-compatibility: Method Invocation with :

- $\sigma \in \mathcal{D} \llbracket \Delta \rrbracket$

- $\Delta \vdash T_{1} \triangleleft U_{1}$

- $\left(k^{\prime}, v_{1}, v_{2}\right) \in \mathcal{V} \llbracket \sigma\left(T_{1} \triangleleft U_{1}\right) \rrbracket$

- $\Delta \vdash m \in U_{1}$ and $\operatorname{msig}\left(\Delta ; U_{1}, m\right)=\langle X: A$..B $\rangle S_{2} \rightarrow S$

- $\Delta \vdash U^{\prime} \wedge \Delta \vdash U^{\prime} \in A . . B$

- $\left(k^{\prime \prime}, v_{1}^{\prime \prime}, v_{2}^{\prime \prime}\right) \in \mathcal{V} \llbracket \sigma\left(S_{2}\left[U^{\prime} / X\right]\right) \rrbracket$

to obtain: $\left.\left(k^{\prime \prime}, v_{1} \cdot m\left\langle\sigma\left(U^{\prime}\right)\right\rangle\left(v_{1}^{\prime \prime}\right), v_{2} \cdot m\left\langle\sigma\left(U^{\prime}\right)\right\rangle\left(v_{2}^{\prime \prime}\right)\right)\right) \in \mathcal{C} \llbracket \sigma\left(S\left[U^{\prime} / X\right]\right) \rrbracket$

\section{7) Compatibility-Method-Invocation-High:}

Lemma $29\left(\mathrm{Ob}_{\mathrm{SEC}}^{\langle\rangle}\right.$Compatibility-Method-Invocation-High).

Let $S_{1} \triangleq T_{1} \triangleleft U_{1}, S_{2} \triangleq T_{2} \triangleleft U_{2}$

If $\Delta ; \Gamma \vdash e_{1} \approx e_{1}^{\prime}: T_{1} \triangleleft U_{1}$

$\Delta \vdash m \notin U_{1}, \operatorname{msig}\left(\Delta ; T_{1}, m\right)=\langle X: A . . B\rangle S_{2} \rightarrow T \triangleleft U$

$\Delta \vdash U^{\prime} \wedge \Delta \vdash U^{\prime} \in A . . B$

$\Delta ; \Gamma \vdash e_{2} \approx e_{2}^{\prime}: S_{2}\left[U^{\prime} / X\right]$

then $\Delta ; \Gamma \vdash e_{1} \cdot m\left\langle U^{\prime}\right\rangle\left(e_{2}\right) \approx e_{1}^{\prime} \cdot m\left\langle U^{\prime}\right\rangle\left(e_{2}^{\prime}\right): T\left[U^{\prime} / X\right] \triangleleft \top$

Proof. Proof obligations:

1) $\Delta ; \Gamma \vdash e_{1} \cdot m\left\langle U^{\prime}\right\rangle\left(e_{2}\right): T\left[U^{\prime} / X\right] \triangleleft \top$ and $\Delta ; \Gamma \vdash e_{1}^{\prime} \cdot m\left\langle U^{\prime}\right\rangle\left(e_{2}^{\prime}\right): T\left[U^{\prime} / X\right] \triangleleft \top$. Apply rule (TmH).

2) Assuming arbitrary $k, \sigma, \gamma_{1}, \gamma_{2}$ such as: $k \geq 0, \sigma \in \mathcal{D} \llbracket \Delta \rrbracket,\left(k, \gamma_{1}, \gamma_{2}\right) \in \mathcal{G} \llbracket \sigma(\Gamma) \rrbracket$

Show:

$$
\left(k, \sigma\left(\gamma_{1}\left(e_{1} \cdot m\left\langle U^{\prime}\right\rangle\left(e_{2}\right)\right)\right), \sigma\left(\gamma_{2}\left(e_{1}^{\prime} \cdot m\left\langle U^{\prime}\right\rangle\left(e_{2}^{\prime}\right)\right)\right)\right) \in \mathcal{C} \llbracket \sigma\left(T\left[U^{\prime} / X\right]\right) \triangleleft \top \rrbracket
$$

Apply Lemma 21 Well-typed terms are related at top with:

- $\Delta ; \Gamma \vdash e_{1} . m\left\langle U^{\prime}\right\rangle\left(e_{2}\right): T\left[U^{\prime} / X\right] \triangleleft \top$ and $\Delta ; \Gamma \vdash e_{1}^{\prime} \cdot m\left\langle U^{\prime}\right\rangle\left(e_{2}^{\prime}\right): T\left[U^{\prime} / X\right] \triangleleft \top$. It follows from above.

- $\sigma \in \mathcal{D} \llbracket \Delta \rrbracket,\left(k, \gamma_{1}, \gamma_{2}\right) \in \mathcal{G} \llbracket \sigma(\Gamma) \rrbracket$. It follows from above.

Hence, $\left(k, \sigma\left(\gamma_{1}\left(e_{1} \cdot m\left\langle U^{\prime}\right\rangle\left(e_{2}\right)\right)\right), \sigma\left(\gamma_{2}\left(e_{1}^{\prime} \cdot m\left\langle U^{\prime}\right\rangle\left(e_{2}^{\prime}\right)\right)\right)\right) \in \mathcal{C} \llbracket \sigma\left(T\left[U^{\prime} / X\right]\right) \triangleleft \top \rrbracket$ 
8) Compatibility TPmD:

Lemma $30\left(\mathrm{Ob}_{\mathrm{SEC}}^{\langle\rangle}\right.$Compatibility TPmD).

Let $\Delta ; \Gamma \vdash e_{1} \approx e_{1}^{\prime}: T \triangleleft U$

Let $\Delta \vdash m \in U, \operatorname{msig}(\Delta, U, m)=P_{1} \triangleleft * \rightarrow P_{2} \triangleleft *$

Let $\Delta ; \Gamma \vdash e_{2} \approx e_{2}^{\prime}: P_{1} \triangleleft U_{1}$

Let $\operatorname{rdecl}\left(P_{1} \triangleleft U_{1}, P_{2}\right)=P_{2}^{\prime}$

Then $\Delta ; \Gamma \vdash e_{1} \cdot m\left(e_{2}\right) \approx e_{1}^{\prime} \cdot m\left(e_{2}^{\prime}\right): P_{2} \triangleleft P_{2}^{\prime}$

Proof. Proof obligations:

1) $\Delta ; \Gamma \vdash e_{1} \cdot m\left(e_{2}\right): P_{2} \triangleleft P_{2}^{\prime}$ and $\Delta ; \Gamma \vdash e_{1}^{\prime} \cdot m\left(e_{2}^{\prime}\right): P_{2} \triangleleft P_{2}^{\prime}$. Apply rule (TPmD).

2) Assuming arbitrary $k, \sigma, \gamma_{1}, \gamma_{2}$ such as: $k \geq 0, \sigma \in \mathcal{D} \llbracket \Delta \rrbracket,\left(k, \gamma_{1}, \gamma_{2}\right) \in \mathcal{G} \llbracket \sigma(\Gamma) \rrbracket$

Show:

$$
\begin{gathered}
\left(k, \sigma\left(\gamma_{1}\left(e_{1} \cdot m\left(e_{2}\right)\right)\right), \sigma\left(\gamma_{2}\left(e_{1}^{\prime} \cdot m\left(e_{2}^{\prime}\right)\right)\right)\right) \in \mathcal{C} \llbracket \sigma\left(P_{2} \triangleleft P_{2}^{\prime}\right) \rrbracket \\
\equiv\left(k, \sigma\left(\gamma_{1}\left(e_{1}\right)\right) \cdot m\left(\sigma\left(\gamma_{1}\left(e_{2}\right)\right)\right), \sigma\left(\gamma_{2}\left(e_{1}^{\prime}\right)\right) \cdot m\left(\sigma\left(\gamma_{2}\left(e_{2}^{\prime}\right)\right)\right)\right) \in \mathcal{C} \llbracket \sigma\left(P_{2} \triangleleft P_{2}^{\prime}\right) \rrbracket \\
\equiv\left(k, \sigma\left(\gamma_{1}\left(e_{1}\right)\right) \cdot m\left(\sigma\left(\gamma_{1}\left(e_{2}\right)\right)\right), \sigma\left(\gamma_{2}\left(e_{1}^{\prime}\right)\right) \cdot m\left(\sigma\left(\gamma_{2}\left(e_{2}^{\prime}\right)\right)\right)\right) \in \mathcal{C} \llbracket P_{2} \triangleleft P_{2}^{\prime} \rrbracket
\end{gathered}
$$

Instantiate the hypothesis $\Delta ; \Gamma \vdash e_{1} \approx e_{1}^{\prime}: T \triangleleft U$ with $k, \sigma, \gamma_{1}, \gamma_{2}$, hence:

$\left(k, \sigma\left(\gamma_{1}\left(e_{1}\right)\right), \sigma\left(\gamma_{2}\left(e_{1}^{\prime}\right)\right)\right) \in \mathcal{C} \llbracket \sigma(T \triangleleft U) \rrbracket$

Let $k^{\prime} \leq k$ and let $\left(k^{\prime}, v_{1}, v_{2}\right) \in \mathcal{V} \llbracket \sigma(T \triangleleft U) \rrbracket$. By Lemma 19 (Monadic bind we can rewrite the goal to show

$$
\left(k^{\prime}, v_{1} \cdot m\left(\sigma\left(\gamma_{1}\left(e_{2}\right)\right)\right), v_{2} \cdot m\left(\sigma\left(\gamma_{2}\left(e_{2}^{\prime}\right)\right)\right)\right) \in \mathcal{C} \llbracket P_{2} \triangleleft P_{2}^{\prime} \rrbracket
$$

Instantiate the hypothesis $\Delta ; \Gamma \vdash e_{2} \approx e_{2}^{\prime}: P_{1} \triangleleft U_{1}$ with $k, \rho, \gamma_{1}, \gamma_{2}$, hence:

$\left(k, \sigma\left(\gamma_{1}\left(e_{2}\right)\right), \sigma\left(\gamma_{2}\left(e_{2}^{\prime}\right)\right) \in \mathcal{C} \llbracket \sigma\left(P_{1} \triangleleft U_{1}\right) \rrbracket\right.$

Let $k^{\prime \prime} \leq k^{\prime}$ and let $\left(k^{\prime \prime}, v_{1}^{\prime \prime}, v_{2}^{\prime \prime}\right) \in \mathcal{V} \llbracket \sigma\left(P_{1} \triangleleft U_{1}\right) \rrbracket$. By Lemma 19 (Monadic bind we can rewrite the goal to show

$$
\left.\left(k^{\prime \prime}, v_{1} \cdot m\left(v_{1}^{\prime \prime}\right), v_{2} \cdot m\left(v_{2}^{\prime \prime}\right)\right)\right) \in \mathcal{C} \llbracket P_{2} \triangleleft P_{2}^{\prime} \rrbracket
$$

Instantiate $\left(k^{\prime}, v_{1}, v_{2}\right) \in \mathcal{V} \llbracket \sigma(T \triangleleft U) \rrbracket$ with $m, k^{\prime \prime}, v_{1}^{\prime \prime}, v_{2}^{\prime \prime}$. Note that:

- $m \in \sigma(U)$. It follows from $\Delta \vdash m \in U, \sigma \in \mathcal{D} \llbracket \Delta \rrbracket$ and $\Delta \vdash T \triangleleft U$. Then $\operatorname{msig}\left(\bullet, \sigma\left(T_{1}\right), m\right)=P_{1} \triangleleft * \rightarrow P_{2} \triangleleft *$.

- $k^{\prime \prime}<k$ which follows above assumptions.

- $\left(k^{\prime \prime}, v_{1}^{\prime \prime}, v_{2}^{\prime \prime}\right) \in \mathcal{V} \llbracket P_{1} \triangleleft \sigma\left(U_{1}\right) \rrbracket$. Apply Lemma 16 Downward closed/Monotonicity with $\left(k, v_{1}^{\prime \prime}, v_{2}^{\prime \prime}\right) \in \mathcal{V} \llbracket P_{1} \triangleleft \sigma\left(U_{1}\right) \rrbracket$ and $k^{\prime \prime} \leq k$

Hence, $\left(k^{\prime \prime}, v_{1} \cdot m\left(v_{1}^{\prime \prime}\right), v_{2} \cdot m\left(v_{2}^{\prime \prime}\right)\right) \in \mathcal{C} \llbracket P_{2} \triangleleft \operatorname{rdecl}\left(P_{1} \triangleleft \rho\left(U_{1}\right), P_{2}\right) \rrbracket$

Then, note that $\operatorname{rdecl}\left(P_{1} \triangleleft \rho\left(U_{1}\right), P_{2}\right)=\operatorname{rdecl}\left(P_{1} \triangleleft U_{1}, P_{2}\right)=P_{2}^{\prime}$.

Hence, $\left.\left(k^{\prime \prime}, v_{1} \cdot m\left(v_{1}^{\prime \prime}\right), v_{2} \cdot m\left(v_{2}^{\prime \prime}\right)\right)\right) \in \mathcal{C} \llbracket P_{2} \triangleleft P_{2}^{\prime} \rrbracket$

\section{9) Compatibility TPmH:}

Lemma $31\left(\mathrm{Ob}_{\mathrm{SEC}}^{\langle\rangle}\right.$Compatibility TPmH).

Let $\Delta ; \Gamma \vdash e_{1} \approx e_{1}^{\prime}: T \triangleleft U$

Let $\Delta \vdash m \in T$, $\operatorname{msig}(\Delta, T, m)=P_{1} \triangleleft * \rightarrow P_{2} \triangleleft *$

Let $\Delta ; \Gamma \vdash e_{2} \approx e_{2}^{\prime}: P_{1} \triangleleft U_{1}$

Then $\Delta ; \Gamma \vdash e_{1} \cdot m\left(e_{2}\right) \approx e_{1}^{\prime} \cdot m\left(e_{2}^{\prime}\right): P_{2} \triangleleft \top$

Proof. Proof obligations:

1) $\Delta ; \Gamma \vdash e_{1} \cdot m\left(e_{2}\right): P_{2} \triangleleft \top$ and $\Delta ; \Gamma \vdash e_{1}^{\prime} . m\left(e_{2}^{\prime}\right): P_{2} \triangleleft \top$. Apply rule (TPmH).

2) Assuming arbitrary $k, \sigma, \gamma_{1}, \gamma_{2}$ such as: $k \geq 0, \sigma \in \mathcal{D} \llbracket \Delta \rrbracket,\left(k, \gamma_{1}, \gamma_{2}\right) \in \mathcal{G} \llbracket \sigma(\Gamma) \rrbracket$

Show:

$$
\left(k, \sigma\left(\gamma_{1}\left(e_{1} \cdot m\left(e_{2}\right)\right)\right), \sigma\left(\gamma_{2}\left(e_{1}^{\prime} \cdot m\left(e_{2}^{\prime}\right)\right)\right)\right) \in \mathcal{C} \llbracket \sigma\left(P_{2}\right) \triangleleft \top \rrbracket
$$

Apply Lemma 21 Well-typed terms are related at top with:

- $\Delta ; \Gamma \vdash e_{1} \cdot m\left(e_{2}\right): P_{2} \triangleleft \top$ and $\Delta ; \Gamma \vdash e_{1}^{\prime} \cdot m\left(e_{2}^{\prime}\right): P_{2} \triangleleft \top$. It follows from above

- $\sigma \in \mathcal{D} \llbracket \Delta \rrbracket,\left(k, \gamma_{1}, \gamma_{2}\right) \in \mathcal{G} \llbracket \sigma(\Gamma) \rrbracket$. It follows from above.

Hence, $\left(k, \sigma\left(\gamma_{1}\left(e_{1} \cdot m\left(e_{2}\right)\right)\right), \sigma\left(\gamma_{2}\left(e_{1}^{\prime} \cdot m\left(e_{2}^{\prime}\right)\right)\right)\right) \in \mathcal{C} \llbracket \sigma\left(P_{2}\right) \triangleleft \top \rrbracket$ 
10) Fundamental property:

Theorem 3 (Fundamental property).

$\Delta ; \Gamma \vdash e: S \Longrightarrow \Delta ; \Gamma \vdash e \approx e: S$

Proof. The proof is by induction on the typing derivation of $\Delta ; \Gamma \vdash e: S$.

Each case follows directly from the corresponding compatibility lemma (Lemmas 24... 31)

Lemma 2 (Self logical relation implies PRNI).

$\Delta ; \Gamma \vdash e \approx e: S \Longrightarrow \operatorname{PRNI}(\Delta, \Gamma, e, S)$

Proof. The proof is direct given the similarity of both definitions. The only difference between both definitions is that $\Delta, \Gamma \vdash e \approx e: S$ uses the security type system (Figure 5) and $\operatorname{PRNI}(\Delta, \Gamma, e, S)$ uses the simple type system (Figure 23). We use Lemma 8 Well-type programs are simple well-typed to show that $\Delta ; \Gamma \vdash e: T \triangleleft U \Rightarrow \Gamma \vdash_{1} e: T$ 


\section{APPENDIX E \\ LIST OF FIGURES}

LIST OF FIGURES

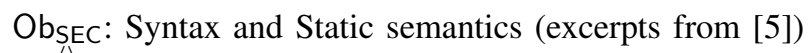

$\mathrm{Ob}_{\mathrm{SEC}}^{\langle\rangle}:$Syntax

$\mathrm{Ob}_{\mathrm{SEC}}^{\langle\rangle}$: Subtyping rules

$\mathrm{Ob}_{\mathrm{SEC}}^{\langle\backslash \mathrm{Y}}$ : Some auxiliary definitions .

$\mathrm{Ob}_{\mathrm{SEC}}^{\langle\backslash}$ : Static semantics .

$\mathrm{Ob}_{S \mathrm{SEC}}$ : Dynamic semantics

$\mathrm{Ob}_{\mathrm{SEC}}^{\mathrm{Y}\rangle \mathrm{S}}$ Step-indexed logical relation for type-based equivalence $\ldots \ldots \ldots \ldots \ldots \ldots$

$\mathrm{Ob}_{\mathrm{SEC}}^{\mathrm{S}}$ : Extended syntax for primitive types $\ldots \ldots \ldots \ldots \ldots \ldots \ldots$

$\mathrm{Ob}_{\mathrm{SEC}}^{(\backslash \mathrm{C}}$ : Subtyping rules for primitive types $\ldots \ldots \ldots \ldots \ldots \ldots \ldots \ldots$

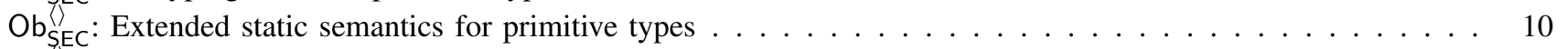

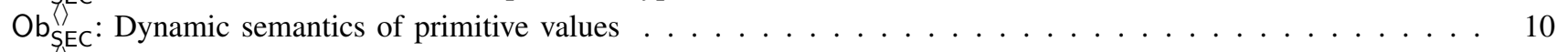

$\mathrm{Ob}_{S E C}^{\backslash \backslash}$ : Step-indexed logical relation with new definitions for primitive types ............ 10

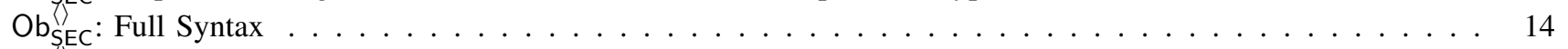

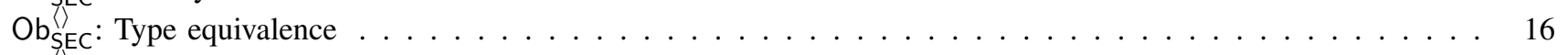

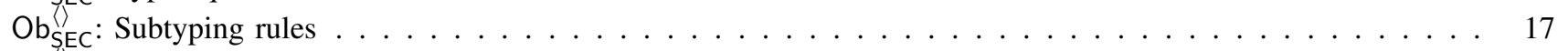

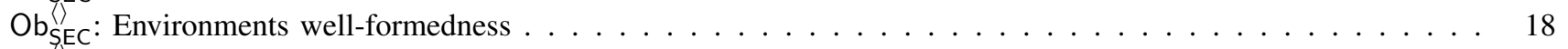

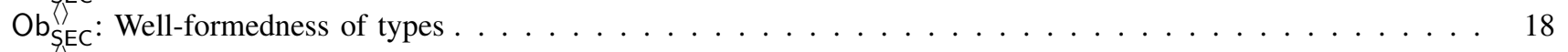

$\mathrm{Ob}_{\mathrm{SEC}}^{\langle\backslash} \mathrm{i}$ Rules for valid relation between facets $\ldots \ldots \ldots \ldots \ldots \ldots \ldots \ldots \ldots$

$\mathrm{Ob}_{\mathrm{SEC}}^{\langle\rangle \mathrm{C}}$ : Well-formedness of security types facet-wise $\ldots \ldots \ldots \ldots \ldots \ldots \ldots \ldots \ldots$

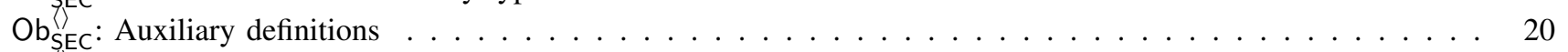

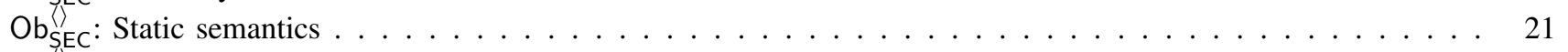

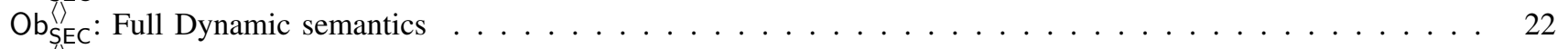

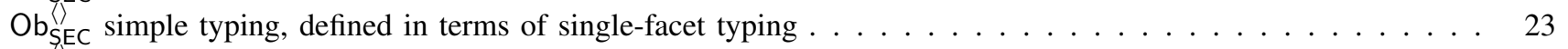

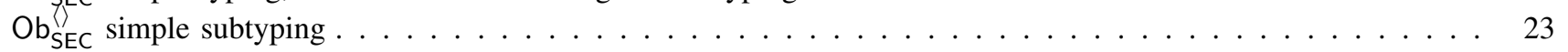

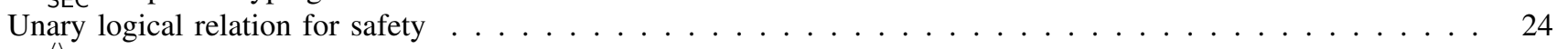

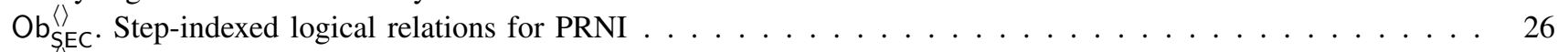

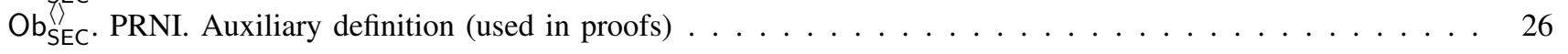

\section{APPENDIX F \\ LIST OF THEOREMS \\ LIST OF THEOREMS}

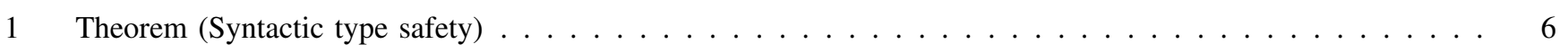

2 Lemma (Self logical relation implies PRNI) $\ldots \ldots \ldots \ldots \ldots \ldots \ldots \ldots$

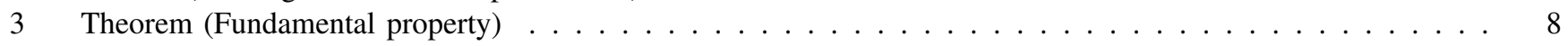

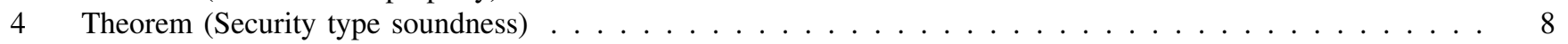

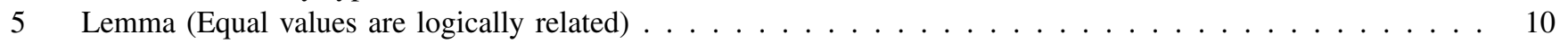

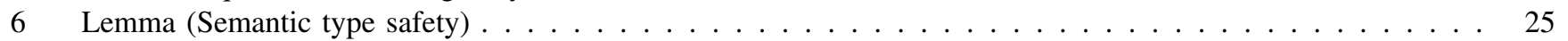

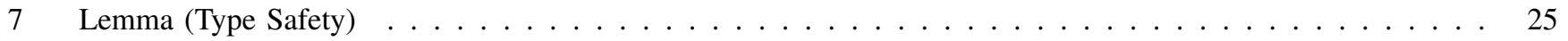

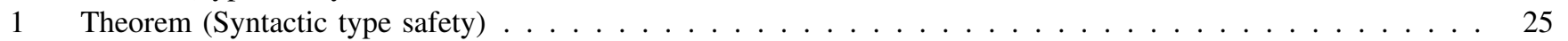

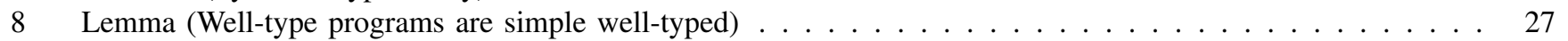

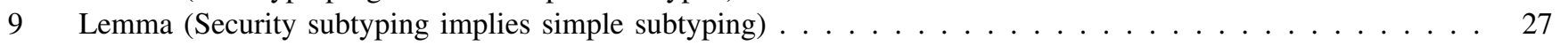

10 Lemma (Value substitution preserves simple typing) $\ldots \ldots \ldots \ldots \ldots \ldots \ldots \ldots \ldots \ldots$

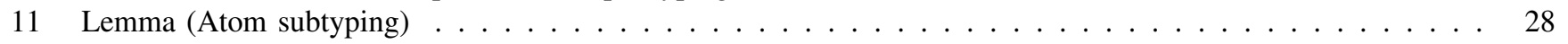

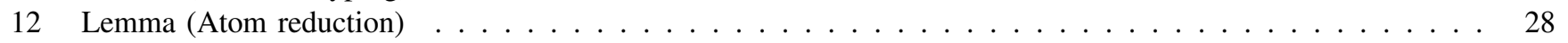

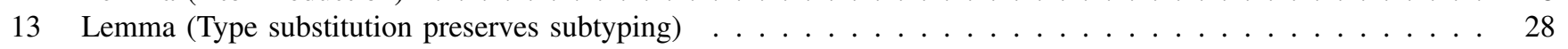

14 Lemma (Type substitution preserves interval subtyping) $\ldots \ldots \ldots \ldots \ldots \ldots \ldots \ldots \ldots$

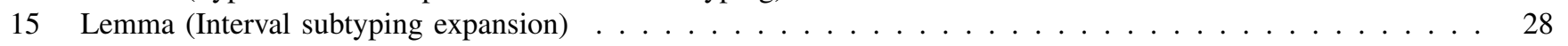

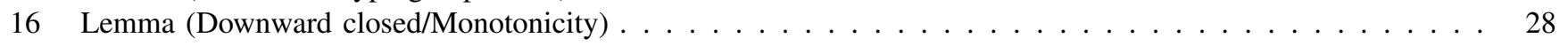

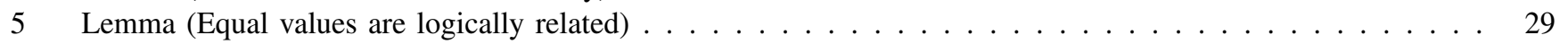




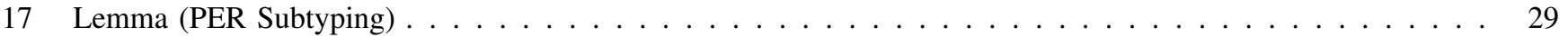

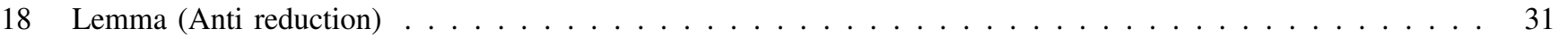

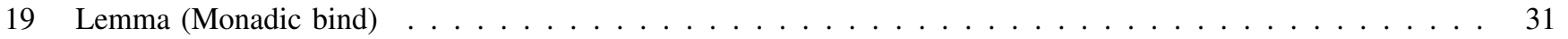

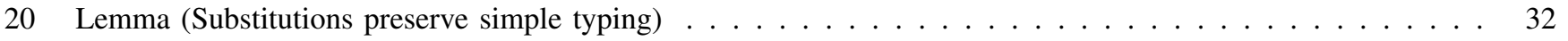

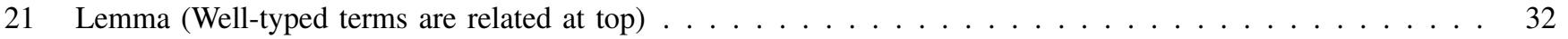

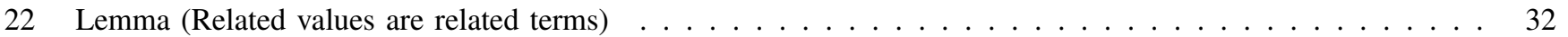

23 Lemma (Pre-compatibility: Method Invocation) . . . . . . . . . . . . . . . . . . . . . . . . 33

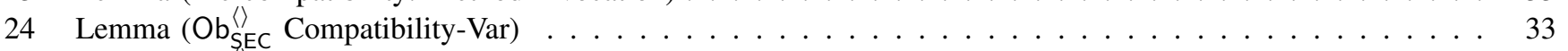

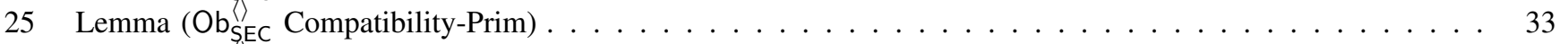

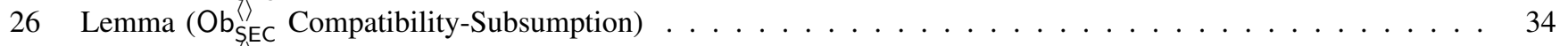

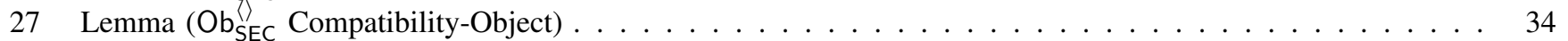

28 Lemma $\left(\mathrm{Ob}_{\mathrm{SEC}}\right.$ Compatibility-Method-Invocation-Declassification $) \ldots \ldots \ldots \ldots \ldots \ldots$

29 Lemma $\left(\mathrm{Ob}_{S E C}^{\backslash\rangle}\right.$ Compatibility-Method-Invocation-High $) \ldots \ldots \ldots \ldots \ldots \ldots$

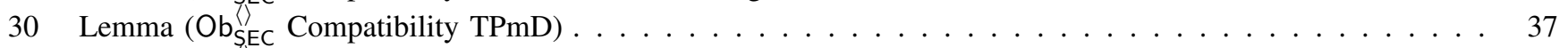

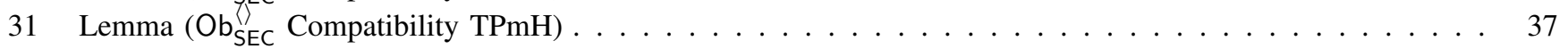

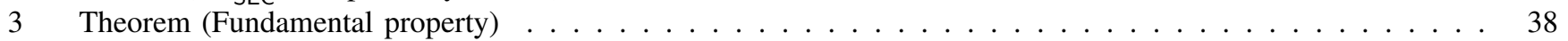

2 Lemma $($ Self $\operatorname{logical}$ relation implies PRNI $\ldots \ldots \ldots \ldots \ldots \ldots \ldots$ 